\title{
Birds of the Pantanal floodplains, Brazil: historical data, diversity, and conservation
}

\begin{abstract}
Alessandro Pacheco Nunes ${ }^{1}$; Sérgio Roberto Posso ${ }^{2}$; Angélica Vilas Boas da Frota ${ }^{3,17}$; Breno Dias Vitorino ${ }^{3},{ }^{18}$; Rudi Ricardo Laps ${ }^{4}$; Reginaldo José Donatelli ${ }^{5}$; Fernando Costa Straube ${ }^{6}$; Maria Antonietta Castro Pivatto ${ }^{7}$; Dalci Maurício Miranda de Oliveira ${ }^{8}$; Braulio Carlos ${ }^{9}$; Alyson Vieira de Melo ${ }^{10}$; Walfrido Moraes Tomas ${ }^{11}$; Gabriel Oliveira de Freitas ${ }^{12}$; Rafael Augusto Ducel de Souza ${ }^{13}$; Maristela Benites $^{14,15,19}$; Simone Mamede ${ }^{15,20}$ \& Renato Soares Moreira ${ }^{16}$
\end{abstract}

1 Bioma Meio Ambiente - Consultoria Ambiental. Corumbá, MS, Brasil. ORCID: http://orcid.org/0000-0003-0031-9938. E-mail: tiriba.ms@gmail.com (corresponding author)

2 Universidade Federal de Mato Grosso do Sul (UFMS), Laboratório de Ecologia, Sistemática e Conservação das Aves Neotropicais (LESCAN). Três Lagoas, MS, Brasil. ORCID: http://orcid.org/0000-0002-7823-9068. E-mail: srposso@hotmail.com

3 Universidade do Estado de Mato Grosso (UNEMAT), Centro de Limnologia, Biodiversidade e Etnobiologia do Pantanal (CELBE), Programa de Pós-Graduação em Ciências Ambientais (PPGCA). Cáceres, MT, Brasil.

${ }^{4}$ Universidade Federal de Mato Grosso do Sul (UFMS), Centro de Ciências Biológicas e da Saúde (CCBS), Programa de Pós-Graduação em Ecologia e Conservação. Campo Grande, MS, Brasil. ORCID: http://orcid.org/0000-0002-2668-9171. E-mail: rudilaps@uol.com.br

${ }^{5}$ Universidade Estadual Paulista "Júlio de Mesquita Filho" (UNESP), Faculdade de Ciências, Departamento de Ciências Biológicas, Laboratório de Ornitologia. Bauru, SP, Brasil. ORCID: http://orcid.org/0000-0002-5988-2226. E-mail: reginaldo.donatelli@unesp.br

${ }^{6}$ HORI Consultoria Ambiental. Curitiba, PR, Brasil. ORCID: http://orcid.org/0000-0002-1881-5000. E-mail: fernando@hori.bio.br

7 A Loja dos Passarinhos, Consultoria em Turismo de Observação de Aves. São Paulo, SP, Brasil. ORCID: http://orcid.org/0000-0003-2499-3062. E-mail: tietta.pivatto@gmail.com

${ }^{8}$ Universidade Federal de Mato Grosso (UFMT), Instituto de Biociências (IB), Departamento de Biologia e Zoologia. Cuiabá, MT, Brasil. ORCID: http://orcid.org/0000-0002-8905-2274. E-mail: acquavis@gmail.com

9 Birding \& Wildlife Safaris. Cuiabá, MS, Brasil. ORCID: http://orcid.org/0000-0002-1717-9938. E-mail: brauliocarlos@gmail.com

${ }^{10}$ Universidade Federal Rural de Pernambuco (UFRPE), Programa de Pós Graduação em Biodiversidade e Conservação (PGBC). Serra Talhada, PE, Brasil. ORCID: http://orcid.org/0000-0003-1659-7801. E-mail: alysonmelo@hotmail.com

${ }^{11}$ Empresa Brasileira de Pesquisa Agropecuária - Pantanal (EMBRAPA Pantanal), Laboratório de Vida Selvagem. Corumbá, MS, Brasil. ORCID: http://orcid.org/0000-0001-9395-7415. E-mail:walfrido.tomas@embrapa.br

${ }^{12}$ Fundação do Meio Ambiente do Pantanal, Educação Ambiental. Cuiabá, MS, Brasil. ORCID: http://orcid.org/0000-0002-4270-903X. E-mail: gabrielrj.freitas@gmail.com

${ }^{13}$ Icterus Ecoturismo e Expedições, Condutor de Observação de Aves. Corumbá, MS, Brasil. ORCID: http://orcid.org/0000-0003-2100-9621. E-mail: rafaelducel@outlook.com

${ }^{14}$ Universidade Federal de Mato Grosso do Sul (UFMS), Centro de Ciências Biológicas e da Saúde (CCBS), Programa de Pós-Graduação em Ensino de Ciências. Campo Grande, MS, Brasil.

${ }^{15}$ Instituto Mamede de Pesquisa Ambiental e Ecoturismo. Campo Grande, MS, Brasil.

${ }^{16}$ Organização de Desenvolvimento Sustentável (ODS). Patos de Minas, MG, Brasil. ORCID: http://orcid.org/0000-0003-0700-8446. E-mail: renatomoreiraods@gmail.com

${ }^{17}$ ORCID: http://orcid.org/0000-0003-3693-4756. E-mail: angelicafrotaa@gmail.com

${ }^{18}$ ORCID: http://orcid.org/0000-0002-5293-8581. E-mail: brenovitorino@gmail.com

${ }^{19}$ ORCID: http://orcid.org/0000-0003-0024-1122. E-mail: maris.benites@gmail.com

${ }^{20}$ ORCID: http://orcid.org/0000-0002-7785-320X. E-mail: institutomamede2@gmail.com

\begin{abstract}
The Pantanal floodplains of Brazil are a region of rich biodiversity. To date, the true richness of the Pantanal avifauna has not been explored satisfactorily caused by a lack of studies in the region and, especially, by the divergence of opinion among the works published by various authors on the many species found in the region. This is due to the lack of criteria in examining records, both with regard to the reliability of the identifications and in the precise geographical allocation. Therefore, in the study, we collage findings from various studies and records created by us in the last few decades from 199 distinct locations to produce a list of birds in the Pantanal floodplains. We grouped the results into three lists: primary, secondary, and tertiary. We found that the avifauna of the Pantanal floodplain is composed of a total of 617 species, of which 571 (92\%) have supporting records of occurrence (primary list) and 46 still lack documentation (secondary list). The number of species listed here for the Pantanal floodplain represents $32 \%$ of all avifauna known to the Brazilian territory. This reflects the importance of the biome, as part of the national territory, for the maintenance of a meaningful avifaunistic richness. Migratory birds $(n=183)$, notably northern ones $(n=43)$, are among the main players involved in ecological processes of nutrient cycling and dispersion of
\end{abstract}


important pathogens between the two continents. With regard to conservation, 25 species are included in some category of threat in the lists of threatened species with global extinction. We hope that our list will help future researchers a more definitive approach when researching the avian fauna in this bountiful region.

Keywords. Birds; Biogeographic distribution; Threatened species; Migration; Wetlands and marshes.

\section{INTRODUCTION}

The Brazilian Pantanal has a National and International recognition as one of the main inland wetlands for its wide territory (Harris et al., 2005) and by provide of multiple ecosystem services, such as the maintenance of biodiversity (Mitsch et al., 2015). The avian occurring in the Pantanal floodplain are associated with habitats diversity that is regulated by the annual and pluriannual flood pulse dynamics (Junk et al., 2006). Thus, the relevance of the Pantanal has been attractive to many naturalists and researchers who focus on elucidate general aspects related to the taxonomy, biogeography and ecology of birds. In 1825, the Austrian naturalist Johann Natterer, coming from the south of Goiás, entered the Pantanal region from Cuiabá on what would be the first scientific expedition to the floodplain in the region of Cáceres, state of Mato Grosso (Tubelis \& Tomas, 2003a, b). Subsequently, Alfredo Borelli and Herbert H. Smith coordinated expeditions around Corumbá and Porto Murtinho in Mato Grosso do Sul (Allen, 1891, 1892, 1893; Salvadori, 1895, 1900; Straube, 2010). In the $20^{\text {th }}$ century, the RooseveltRondon expeditions took place in the former state of Mato Grosso (Naumburg et al., 1930), those of James AG Rehn occurred in Descalvados (Stone \& Roberts, 1934), and those of the Zoology Museum of the University of São Paulo were located in some locations in the Pantanal region (Pinto, 1932, 1938, 1940, 1944). Between 1940 and 1960, expeditions were carried out by national institutions in the north, the center, and along the meridian of the Pantanal floodplain (Tubelis \& Tomas, 2003b). Their observations and collections resulted in relevant publications on the species found in the region (Travassos, 1940, 1941; Travassos \& Freitas, 1942; Pinto, 1948; Ruschi, 1955; Travassos et al., 1957; Sick, 1961). In 1970, specimen collections were made by the Museum of Fauna (formerly Brazilian Institute of Forestry Development) from Fazenda Descalvados, Mato Grosso (Tubelis \& Tomas, 2003b), and a multitude of contemporary authors such as Antas (1983), Munn et al. (1989), Yamashita \& Valle (1990), Guedes (1993), Antas (1994), Willis (1995), Guedes \& Harper (1995), Antas \& Nascimento (1996), Oliveira (1997), Yamashita (1997), Pinho (1998), and Tubelis \& Tomas (1999) focused on the biology of species and communities through capture, direct observation, and identification of vocalizations (Tubelis \& Tomas, 2003b). Additional inventories and records were collated by Weinberg (1984), Antas et al. (1986), Cintra \& Yamashita (1990), Willis \& Oniki (1990), Lago-Paiva \& Willis (1994), Pacheco \& Bauer (1994), and Strüssmann (1998).

Although the contribution of ornithological information about this vast perimeter is considered reasonable for an understanding of the records and distribution patterns, there is still much information from other ornithological initiatives carried out in the region that has not been adequately catalogued or considered. The gaps begin with Georg von Langsdorff and his great expedition (1826-1828), whose results, although known to exist, are considered of minimal impact. Among others, the collections of Emilie Snethlage in the region of Corumbá in 1928 and of Alexander Daveron along the Paraguay River (including the region of Cáceres) in 1931, each representing an impressive number of specimens that are maintained to this day by the Museum National of Rio de Janeiro (UFRJ) and the United States National Museum (Smithsonian Institution), respectively, stand out as deserving of an in-depth analysis (Lopes et al., 2016).

A large part of the true richness of the Pantanal avifauna remains unknown due to the lack of studies in many newly undiscovered regions and, especially, by the divergence of opinion among the various researchers working in the region. These occur largely due to the lack of criteria in examining records, both regard to the reliability of the identifications and in the precise geographical allocation of a species.

Brown-Jr. (1986) prepared the first list of birds found in the Upper Paraguay Basin, counting 309 species, and Coutinho et al. (1997) increased this number to 656 species for the Pantanal. However, in both cases, the locations were not georeferenced and the species occurring in the adjacent plateaus were also included in the lists, a fact that made geographic retrieval and data measurement impossible.

Subsequently, Tubelis \& Tomas (2003a) developed a robust and judicious 463 species list, based on records available in the literature, specimens deposited in museums in Brazil and abroad, and new field data. Junk et al. (2006) mentioned 766 bird species in the Pantanal region, 390 of which are considered to be currently present in the floodplain; however, other records do not present adequate supporting documentation for this claim. A list generated by the Brazilian Institute of Environmental and Renewal Energy Resources (IBAMA, 2007) based solely on data published by Brown-Jr. (1986) while disregarding other available publications, indicates only 290 species for the Pantanal region, thereby severely underestimating the diversity of birds occurring in the floodplains.

According to Nunes et al. (2008), if one considers all available data for Pantanal avifauna, the number of species can exceed 730. However, these authors only considered 553 species for the Pantanal region, with a caveat that the occurrence of at least 200 species (27.4\%) is questionable due to its distribution restriction on adjacent plateaus. Tomas et al. (2008) mentioned more 
than 800 species of birds in the Upper Paraguay Basin (Brazil, Bolivia, and Paraguay) of which, at least, 567 had already been recorded in the Brazilian Pantanal region. Petermann (2010) points out between 469 and 522 species for the Pantanal region, whereas Nunes (2011a) related the occurrence of 582 species for the lowland.

Field guides, such as that by Gwynne et al. (2010), indicate a richness of 740 species of birds in central Brazil and, among these, 496 were credited by these authors to the Pantanal region. Pivatto \& Bernardon (2012) and Cintra (2014) published guides for the identification of Pantanal avifauna and listed 455 and 523 species for the region, respectively.

Despite being relevant contributions, most of the publications cited here have questionable records and, in some cases, are based on vague geographical limits, thereby making it difficult the understanding of birds occurring in the Pantanal. Thus, in this study, we aim to present a consolidated list of birds for the lowland of the Brazilian Pantanal, obtained through data collection in the field as well as through the compilation and review of a wide range of reliable historical and contemporary records to provide a safer reference list.

\section{MATERIAL AND METHODS}

\section{The Pantanal floodplains: location, landscape, and environment}

The Pantanal is one of the largest continuous wetlands on the planet. It is located in the center of South America and is cover over more than $140,000 \mathrm{~km}^{2}$ across the Upper Paraguay River Basin and its tributaries that drain the Cerrado region of Central Brazil, and areas of Bolivia's Chaco region and Paraguay (Harris et al., 2005). In Brazil, the Pantanal extends for approximately $250 \mathrm{~km}$ in an east-west direction and $450 \mathrm{~km}$ in a north-south direction and, despite several elevations that exist inside, many of them isolated, the altitude varies only from 60 to $150 \mathrm{~m}$ (Hamilton et al., 1996).

The landscape in the floodplain of the Brazilian Pantanal is complex, diversified, and occurs in mosaics with diverse phytophysiognomies and ecological situations that are ordered by topographic gradients and flood pulses (Junk et al., 2014). Natural vegetation of the region is influenced by the adjacent phytogeographic provinces such as Cerrado (Brazilian savanna), Chaco (dry woodland or dry forest), Amazon and Atlantic Forest (Nunes \& Tomas, 2004a; Silva \& Santos, 2005).

The coverage and land use of the Pantanal region is currently made up of natural non-forest formations (grasslands and marshes of perennial or seasonal vegetation) and forest (savanna and seasonal forest) to spaces used by agriculture (pastures and monocultures), in addition to various bodies of water of different natures (Miranda et al., 2017; Souza-Jr. et al., 2020). All these landscape units are regulated by annual and multi-annual rain cycles that intersect with rainy and dry periods and promote extreme events of floods and droughts, the latter often being combined with large fires (Nunes Da Cunha \& Junk, 2004). In general, the Pantanal climate is predominantly hot and humid, with an average annual rainfall of $1,500 \mathrm{~mm}$ and a maximum average temperature of $32^{\circ} \mathrm{C}$ and a minimum of $20^{\circ} \mathrm{C}$ (Alvares et al., 2014).

In this study, we considered the area recognized as the floodplain of the Brazilian Pantanal region (Fig. 1) within whose limits the respective records of the occurrence of the various bird species were plotted. It encompasses a perimeter of $150,000 \mathrm{~km}^{2}$ exclusively in the Brazilian territory, $4.6 \%$ of which is represented by protected areas including 18 private reserves, 1 national park, 3 state parks, 1 municipal park, 1 environmental protection area, and 1 ecological station (Brazil, 2020). For the elaboration of our database, 199 locations in the states of Mato Grosso and Mato Grosso do Sul that presented one or more records of avifauna were evaluated.

\section{Data collection}

For the elaboration of the consolidated list of birds of the Pantanal floodplain, we considered the records created by us in the last decades, whether from systematic samplings or not: Allen (1891, 1892, 1893); Naumburg et al. (1930); Naumburg (1935); Zimmer (1933); Jesus \& Lima (2003); Antas \& Palo-Jr. (2004); Donatelli (2005); Pinho (2005); Cestari (2006a, b); Straube et al. (2006a, b; 2007); Melo et al. (2007); Mestre (2007); Straube et al. (2007); Amaral \& Ragusa-Netto (2008); Donatelli \& Ubaid (2008); Melo \& Teribeli (2008); Nunes et al. (2008); Pivatto et al. (2008); Ubaid \& Donatelli (2008); Vasconcelos et al. (2008); Wittaker et al. (2008); Antas \& Palo-Jr. (2009); Chiaravalloti et al. (2009); Nunes et al. (2009); Mestre et al. (2010); Nunes (2010); Nunes et al. (2010); Evangelista et al. (2010); Serrano (2010); Signor \& Pinho (2010); Ubaid et al. (2010); Yabe et al. (2010); Brandão et al. (2011); Nunes (2011a, b); Nunes et al. (2011); Leuzinger (2011); Ribas et al. (2011); Straube \& Melo (2011); Emanuel (2013); Nunes et al. (2013); Kantek \& Onuma (2013); Ubaid \& Antas (2013); Donatelli et al. (2014); Nunes (2015); SeveroNeto et al. (2015); Lopes et al. (2016); Benites et al. (2017); Donatelli et al. (2017); Severo-Neto et al. (2017); Vitorino et al. (2017); Nabuco et al. (2018); Nunes et al. (2018); Frota et al. (2020a) and Benites \& Mamede (in press). In all, 199 locations were visited, 29 of which could not be georeferenced. The information on these locations, period of observations, and authors of the records can be accessed in Table S1. Also, the occurrences are mentioned in the literature (Rv/sP), in the authors' field works (Rv/sNP), and in the form of specimens deposited in museums and institutional ornithological collections in Brazil and abroad (ExM). In addition, data in the form of photographic records deposited in institutional museums (FoM), cited in scientific journals (FoP) or private collection (FoAP), photographic records (Fol) and sound (ASI) records available on internet data platforms, audiovisual (ViM) and sound records (ASM) deposited in institutional museums, and sound records (ASI) deposited in data platforms are available for online consultation (Carlos et al., 2010). 


\section{Bird records in the Brazilian Pantanal}
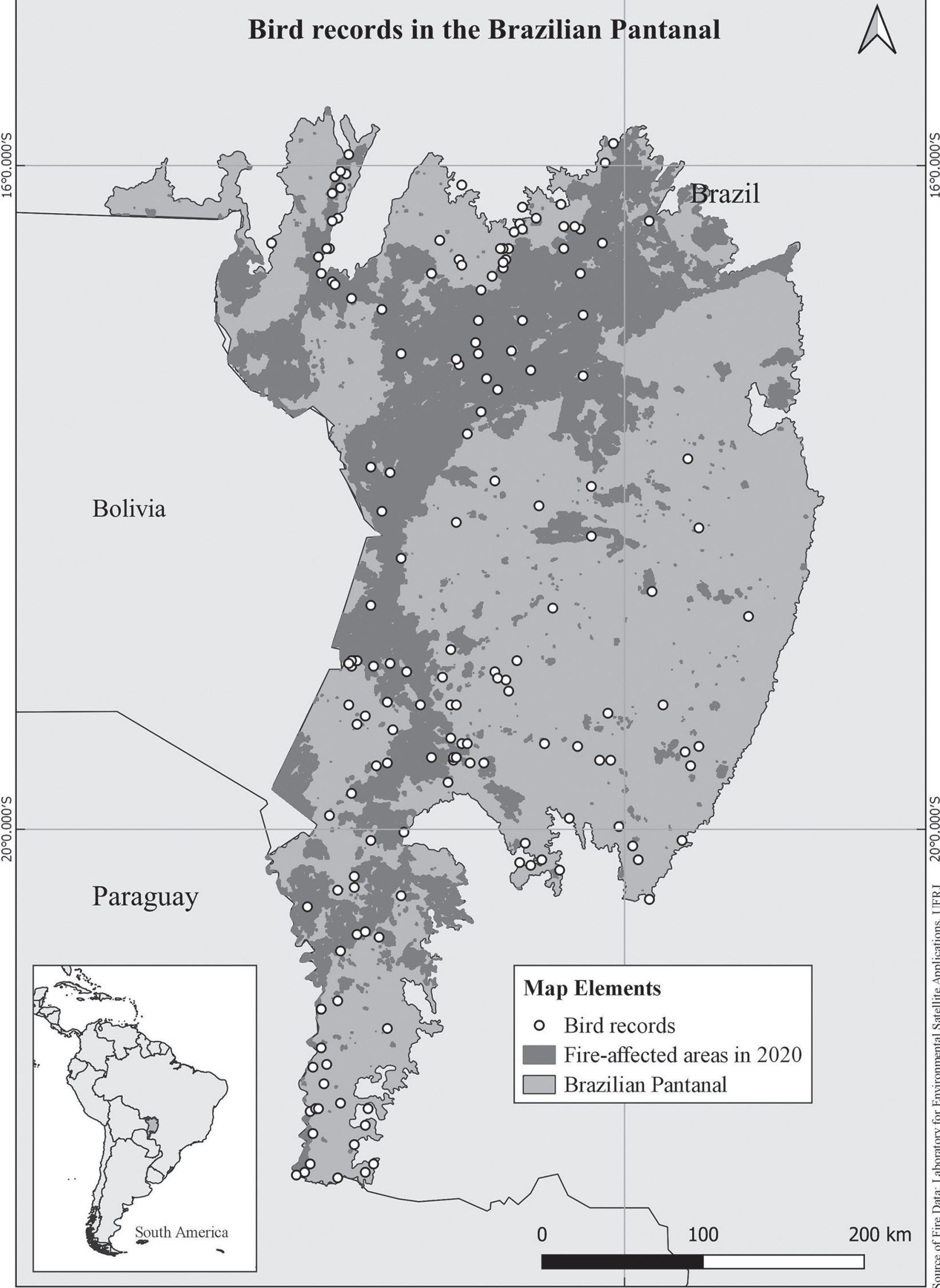

$56^{\circ} 0.000^{\prime} \mathrm{W}$

Figure 1. Localities with ornithological inventories in Pantanal wetland. The circles correspond to different sample sites, whose geographic coordinates can be found in Table S1. The dark gray spots correspond to burned areas in 2020 fire gray spots correspond to burned areas in 2020 fire according to ALARMES-HISTÓRICO (LASA/ UFRJ, 2021; Pinto et al., 2020). 
Data on specimens deposited in institutional museums in Brazil and abroad were obtained by consulting Tubelis \& Tomas (2003a) and the website (Vertnet, 2021). Sound archives deposited in institutional museums obtained from the Arquivo Sonoro Prof. Elias Coelho (ASEC), from the Federal University of Rio de Janeiro and at the Neotropical Fonoteca Jacques Vielliard (FNJV) of the Zoology Museum of the State University of Campinas. Regarding the photographic and sound records available on digital media, we evaluated those hosted until Mach 2021 in the WikiAves (WikiAves, 2021 - http://www.wikiaves.com), Xeno-canto (Xeno-Canto, 2021 - https://www.xeno-canto/databases. org), and Visual Resources of Ornithology/VIREO (VIREO, 2021 - http://vireo.ansp.org) databases. Records in municipalities that include stretches of plateau (e.g., Corumbá, Miranda, Aquidauana, Coxim, Rio Verde de Mato Grosso, Cáceres, and Santo Antônio do Leverger) were carefully investigated by confirming their locations with the authors to ensure that only records collected from the floodplain were included in the study. When such records were cited in publications without specific details on location, the species were not included in the main list.

\section{Data requirements for inclusion into inventory lists}

We adopted the taxonomic classification proposed by the Brazilian Ornithological Records Committee (Pacheco et al. 2021). The primary list, which was composed of species with at least one occurrence record in the Pantanal floodplain provided with documentary evidence, i.e., item/(s) available for independent consultation as a full or partial specimen, photograph, and audio/ video recording, which allow the safe and indisputable determination of the taxon. The secondary list includes species with one or more visual and/or sound records in the Pantanal floodplain, but whose documentary evidence is unknown or unavailable. In this context, the filtering protocol is the detailing of the information allusive to the record, necessarily associated with consistency of the distribution and dispersion patterns of the species based on documentary evidence. The primary and secondary lists constitute the main list.

In addition to extinct taxa of nature or those not validated as full species, some species that, although mentioned in some of the consulted sources, presented questionable records with incompatible distribution and/or occurrence only marginal to the Pantanal floodplain were excluded and are present in the tertiary list. In brief, the main list includes only the species that have been proven to occur within the defined geographical limits, based on the following superior evidence, in hierarchical order: ExM, FoM, FoP, Fol, ViM, ASM, and ASI (for details, see Carlos et al., 2010).

\section{Species categorization}

The species were categorized according to their conservation status based on the global redlist (Handbook of the Birds of the World \& BirdLife International, 2020): Deficient Data (DD), Near Threatened (NT), vulnerable (VU), Endangered (EN), and Critically endangered (CR). Also, we considering the National lists of threatened species (ICMBio, 2014). In addition, we highlight species that show a global population decline (Handbook of the Birds of the World \& BirdLife International, 2020). Based on the lists of migratory birds proposed by Nunes \& Tomas (2008) and Somenzari et al. (2018), the species were initially distinguished as intercontinental (INTER) or intracontinental (INTRA) migrants. The former includes species that make large displacements from the northern hemisphere (Canada and northern USA) towards southern South America (Patagonia). The latter category includes species that move from the southernmost portions of South America towards its northernmost regions (mainly Amazonia) and vice versa.

\section{RESULTS AND DISCUSSION}

According to our study, the avifauna of the Pantanal floodplain is composed of a total of 617 species, of which 571 (92\%) have supporting records of occurrence (primary list) and 46 still lack documentation (secondary list) (Tables 1 and S1). In the tertiary list, we include 63 species whose records were based questionable information or incompatible with the floodplain region (Table 2). Of the migratory species occurring in Brazil, 183 species use the Pantanal floodplain during migratory movements. Most are composed of southern migrants (140 species), especially those moving from the southern portions of South America towards Central Brazil and the Amazon. In this group, the most important species belong to the family Tyrannidae (37 species), genus Sporophila (15 species), and family Hirundinidae (7 species). The northern migrants add up to 43 species, almost half (45\%) the number being formed by members of family Scolopacidae. Species linked to aquatic environments account for $38 \%$ of the migrant species and those dependent on native grasslands in good condition account for $12 \%$ ( 21 species).

With regard to conservation, 25 species are included in some category of threat in the lists of threatened species with global extinction (Handbook of the Birds of the World \& BirdLife International, 2020); of these, four are classified as "Endangered" and "Critically Endangered". In the National territory (ICMBio, 2014), three and two species were classified as "Endangered" and "Critically Endangered", respectively. In the global and national lists, 22 and 13 species are considered "Near Threatment", respectively. In addition, 13 species are inserted in the category "Data Deficient", which reinforces the unknown situation of their populations that are possibly subject to numerous types of threats.

The number of species listed here for the Pantanal wetland represents $32 \%$ of all avifauna known to the Brazilian territory (Pacheco et al., 2021). In the present study, 153 species were addrd to the previous list proposed by Tubelis \& Tomas (2003a). This reflects the importance of the biome, as part of the national terri- 
Table 1. Main list of bird species occurring in the Pantanal, Brazil. Taxa highlighted in square brackets are on the secondary list and lack adequate supporting documentation. Conservation status: * (globally declining population), NT (Near Threatened with extinction), VU (Vulnerable), EN (Endangered), CR (Critically Endangered), PE (Probably extinct), DD (Data Deficient), GL (Global List/Handbook of the Birds of the World \& BirdLife International, 2020), BL (Brazilian List/ICMBio, 2014). Status of migration: INTRA (intracontinental migrant), INTER (intercontinental migrant). Evidence: ExM (specimen deposited in a national institutional museum and/or abroad), Rv/sP (visual and/or sound records quoted in a scientific journal), Rv/sNP (visual and/or sound records obtained by the authors and not published), Fol (photo published on the internet), FoAP (photo in particular collection); ASI (sound file available on the internet).

\begin{tabular}{|c|c|c|c|}
\hline \multirow{2}{*}{ Taxon } & \multicolumn{2}{|c|}{ Status } & \multirow{2}{*}{ Evidence } \\
\hline & Conservation & Migration & \\
\hline \multicolumn{4}{|l|}{ Rheiformes } \\
\hline \multicolumn{4}{|l|}{ Rheidae } \\
\hline Rhea americana (Linnaeus, 1758) & ${ }^{*} \mathrm{NT}(\mathrm{GL})$ & & ExM, Rv/sP, Rv/sNP, Fol, ASI \\
\hline \multicolumn{4}{|l|}{ Tinamiformes } \\
\hline \multicolumn{4}{|l|}{ Tinamidae } \\
\hline Tinamus tao Temminck, 1815 & ${ }^{*} \mathrm{VU}(\mathrm{GL}), \mathrm{VU}(\mathrm{BL})$ & & ASI \\
\hline Crypturellus undulatus (Temminck, 1815) & * & & ExM, Rv/sP, Rv/sNP, Fol, ASI \\
\hline Crypturellus parvirostris (Wagler, 1827) & * & & ExM, Rv/sP, Rv/sNP, Fol \\
\hline Crypturellus tataupa (Temminck, 1815) & $*$ & & $\mathrm{ExM}, \mathrm{Rv} / \mathrm{sP}, \mathrm{Rv} / \mathrm{sNP}, \mathrm{Fol}$ \\
\hline Rhynchotus rufescens (Temminck, 1815) & & & $\mathrm{ExM}, \mathrm{Rv} / \mathrm{sP}, \mathrm{Rv} / \mathrm{sNP}, \mathrm{Fol}$ \\
\hline Nothura maculosa (Temminck, 1815) & * & & ExM, Rv/sP \\
\hline \multicolumn{4}{|l|}{ Anseriformes } \\
\hline \multicolumn{4}{|l|}{ Anhimidae } \\
\hline Anhima cornuta (Linnaeus, 1766) & * & & $\mathrm{Rv} / \mathrm{sP}, \mathrm{Rv} / \mathrm{sNP}$, Fol \\
\hline Chauna torquata (0ken, 1816) & & & ExM, Rv/sP, Rv/sNP, Fol, ASI \\
\hline \multicolumn{4}{|l|}{ Anatidae } \\
\hline Dendrocygna bicolor (Vieillot, 1816) & $*$ & INTRA & ExM, Rv/sP, Fol \\
\hline Dendrocygna viduata (Linnaeus, 1766) & & INTRA & ExM, Rv/sP, Rv/sNP, Fol, ASI \\
\hline Dendrocygna autumnalis (Linnaeus, 1758) & & INTRA & ExM, Rv/sP, Rv/sNP, Fol, ASI \\
\hline Coscoroba coscoroba (Molina, 1782) & & INTRA & $\mathrm{ExM}, \mathrm{Rv} / \mathrm{sP}, \mathrm{Rv} / \mathrm{sNP}, \mathrm{Fol}$ \\
\hline Neochen jubata (Spix, 1825) & *NT (GL), DD (BL) & INTRA & ExM, Rv/sP, Fol \\
\hline Cairina moschata (Linnaeus, 1758) & * & INTRA & ExM, Rv/sP, Rv/sNP, Fol, ASI \\
\hline Sarkidiornis sylvicola lhering \& Ihering, 1907 & * & INTRA & ExM, Rv/sP, Rv/sNP, Fol \\
\hline Callonetta leucophrys (Vieillot, 1816) & & INTRA & $\mathrm{Rv} / \mathrm{sP}, \mathrm{FoP}, \mathrm{Fol}$ \\
\hline Amazonetta brasiliensis (Gmelin, 1789) & * & & ExM, Rv/sP, Rv/sNP, FoP, Fol \\
\hline Spatula versicolor (Vieillot, 1816) & & INTRA & $\mathrm{Rv} / \mathrm{sP}, \mathrm{Fol}$ \\
\hline Spatula platalea (Vieillot, 1816) & & INTRA & $\mathrm{Rv} / \mathrm{sP}, \mathrm{Fol}$ \\
\hline Anas bahamensis Linnaeus, 1758 & * & INTRA & $\mathrm{Rv} / \mathrm{sP}, \mathrm{Fol}$ \\
\hline Netta peposaca (Vieillot, 1816) & & INTRA & $\mathrm{Rv} / \mathrm{sP}, \mathrm{Fol}$ \\
\hline Nomonyx dominicus (Linnaeus, 1766) & * & & ExM, Rv/sP, Fol \\
\hline Oxyura vittata (Philippi, 1860) & & INTRA & ExM, Rv/sP, FoP, Fol \\
\hline \multicolumn{4}{|l|}{ Galliformes } \\
\hline \multicolumn{4}{|l|}{ Cracidae } \\
\hline Penelope superciliaris Temminck, 1815 & $*$ & & ExM, Rv/sP, Fol \\
\hline Penelope ochrogaster Pelzeln, 1870 & *VU (GL), $\mathrm{VU}(\mathrm{BL})$ & & ExM, Rv/sP, Rv/sNP, Fol, ASI \\
\hline Aburria cumanensis (Jacquin, 1784) & *NT (GL) & & ExM, Rv/sP, Rv/sNP, Fol, ASI \\
\hline Aburria cujubi (Pelzeln, 1858) & ${ }^{*} \mathrm{NT}(\mathrm{BL})$ & & ExM, Rv/sP, Rv/sNP, Fol, ASI \\
\hline Ortalis canicollis (Wagler, 1830) & $*$ & & ExM, Rv/sP, Rv/sNP, Fol, ASI \\
\hline Crax fasciolata Spix, 1825 & * VU (GL) & & ExM, Rv/sP, Rv/sNP, Fol, ASI \\
\hline Pauxi tuberosa (Spix, 1825) & & & Fol \\
\hline \multicolumn{4}{|l|}{ Podicipediformes } \\
\hline \multicolumn{4}{|l|}{ Podicipedidae } \\
\hline [Rollandia rolland (Quoy \& Gaimard, 1824)] & * & INTRA & $\mathrm{Rv} / \mathrm{sP}$ \\
\hline Tachybaptus dominicus (Linnaeus, 1766) & & INTRA & ExM, Rv/sP, Fol \\
\hline Podilymbus podiceps (Linnaeus, 1758) & & INTRA & $\mathrm{Rv} / \mathrm{sP}, \mathrm{Fol}$ \\
\hline Podicephorus major (Boddaert, 1783) & & INTRA & Fol \\
\hline \multicolumn{4}{|l|}{ Columbiformes } \\
\hline \multicolumn{4}{|l|}{ Columbidae } \\
\hline Columba livia Gmelin, 1789 & & & $\mathrm{Rv} / \mathrm{sP}, \mathrm{Rv} / \mathrm{sNP}$, Fol \\
\hline Patagioenas speciosa (Gmelin, 1789) & & & ExM, Rv/sP, ASI \\
\hline Patagioenas picazuro (Temminck, 1813) & & INTRA & ExM, Rv/sP, Rv/sNP, Fol, ASI \\
\hline Patagioenas cayennensis (Bonnaterre, 1792) & & & ExM, Rv/sP, Rv/sNP, Fol, ASI \\
\hline [Patagioenas subvinacea (Lawrence, 1868)] & ${ }^{*} \mathrm{VU}(\mathrm{GL})$ & & $\mathrm{Rv} / \mathrm{sP}$ \\
\hline
\end{tabular}




\begin{tabular}{|c|c|c|c|}
\hline \multirow{2}{*}{ Taxon } & \multicolumn{2}{|c|}{ Status } & \multirow{2}{*}{ Evidence } \\
\hline & Conservation & Migration & \\
\hline Geotrygon montana (Linnaeus, 1758) & * & & $\mathrm{Rv} / \mathrm{sP}, \mathrm{Rv} / \mathrm{sNP}$, FoAP \\
\hline Leptotila verreauxi Bonaparte, 1855 & & & ExM, Rv/sP, Rv/sNP, Fol, ASI \\
\hline Leptotila rufaxilla (Richard \& Bernard, 1792) & * & & ExM, Rv/sP, Rv/sNP, Fol, ASI \\
\hline Zenaida auriculata (Des Murs, 1847) & & INTRA & ExM, Rv/sP, Rv/sNP, Fol \\
\hline Claravis pretiosa (Ferrari-Perez, 1886) & & & ExM, Rv/sP, Rv/sNP, Fol \\
\hline Uropelia campestris (Spix, 1825) & & & ExM, Rv/sP, Rv/sNP, Fol, ASI \\
\hline Columbina minuta (Linnaeus, 1766) & & & ExM, Rv/sP, Rv/sNP, Fol \\
\hline Columbina talpacoti (Temminck, 1811) & & & ExM, Rv/sP, Rv/sNP, Fol \\
\hline Columbina squammata (Lesson, 1831) & & & ExM, Rv/sP, Rv/sNP, Fol, ASI \\
\hline Columbina picui (Temminck, 1813) & & & ExM, Rv/sP, Rv/sNP, Fol \\
\hline \multicolumn{4}{|l|}{ Cuculiformes } \\
\hline \multicolumn{4}{|l|}{ Cuculidae } \\
\hline Guira guira (Gmelin, 1788) & & & ExM, Rv/sP, Rv/sNP, Fol, ASI \\
\hline Crotophaga major Gmelin, 1788 & & & ExM, Rv/sP, Rv/sNP, Fol, ASI \\
\hline Crotophaga ani Linnaeus, 1758 & * & & ExM, Rv/sP, Rv/sNP, Fol, ASI \\
\hline Tapera naevia (Linnaeus, 1766) & & & ExM, Rv/sP, Rv/sNP, Fol, ASI \\
\hline Dromococcyx phasianellus (Spix, 1824) & * & & ExM, Rv/sP, Rv/sNP, \\
\hline Dromococcyx pavoninus Pelzeln, 1870 & * & & $\mathrm{Rv} / \mathrm{sP}, \mathrm{Rv} / \mathrm{sNP}$, Fol, ASI \\
\hline Coccycua minuta (Vieillot, 1817) & & & ExM, Rv/sP, Rv/sNP, Fol, ASI \\
\hline Micrococcyx cinereus (Vieillot, 1817) & * & INTRA & $\mathrm{Rv} / \mathrm{sP}, \mathrm{Rv} / \mathrm{sNP}$, Fol \\
\hline Piaya cayana (Linnaeus, 1766) & & & ExM, Rv/sP, Rv/sNP, Fol, ASI \\
\hline Coccyzus melacoryphus Vieillot, 1817 & & INTRA & ExM, Rv/sP, Rv/sNP, Fol, ASI \\
\hline Coccyzus americanus (Linnaeus, 1758) & * & INTER & $\mathrm{Rv} / \mathrm{sP}, \mathrm{Rv} / \mathrm{sNP}$, Fol \\
\hline Coccyzus euleri Cabanis, 1873 & & INTRA & $\mathrm{Rv} / \mathrm{sP}, \mathrm{Rv} / \mathrm{sNP}$, Fol \\
\hline \multicolumn{4}{|l|}{ Nyctibiiformes } \\
\hline \multicolumn{4}{|l|}{ Nyctibiidae } \\
\hline Nyctibius grandis (Gmelin, 1789) & * & & ExM, Rv/sP, Rv/sNP, Fol, ASI \\
\hline Nyctibius griseus (Gmelin, 1789) & * & & ExM, Rv/sP, Rv/sNP, Fol \\
\hline \multicolumn{4}{|l|}{ Caprimulgiformes } \\
\hline \multicolumn{4}{|l|}{ Caprimulgidae } \\
\hline [Nyctiphrynus ocellatus (Tschudi, 1844)] & * & & $\mathrm{Rv} / \mathrm{sP}$ \\
\hline Antrostomus rufus (Boddaert, 1783) & * & INTRA & ExM, Rv/sP, Rv/sNP, Fol, ASI \\
\hline Lurocalis semitorquatus (Gmelin, 1789) & & INTRA & $\mathrm{Rv} / \mathrm{sP}, \mathrm{Fol}$ \\
\hline Nyctiprogne leucopyga (Spix, 1825) & & & ExM, Rv/sP, Rv/sNP, Fol, ASI \\
\hline Nyctidromus albicollis (Gmelin, 1789) & & & ExM, Rv/sP, Rv/sNP, Fol, ASI \\
\hline Hydropsalis parvula Gould, 1837 & & & ExM, Rv/sP, Rv/sNP, Fol, ASI \\
\hline Hydropsalis maculicaudus (Lawrence, 1862) & & & $\mathrm{Rv} / \mathrm{sP}, \mathrm{Rv} / \mathrm{sNP}$, Fol, ASI \\
\hline Hydropsalis torquata (Gmelin, 1789) & & & ExM, Rv/sP, Rv/sNP, Fol \\
\hline Nannochordeiles pusillus (Gould, 1861) & & INTRA & ExM, Rv/sP \\
\hline Podager nacunda (Vieillot, 1817) & & INTRA & ExM, Rv/sP, Rv/sNP, Fol, ASI \\
\hline Chordeiles minor (Forster, 1771) & * & INTER & $\mathrm{Rv} / \mathrm{sP}, \mathrm{Fol}$ \\
\hline [Chordeiles acutipennis (Hermann, 1783)] & & INTRA & $\mathrm{Rv} / \mathrm{sP}$ \\
\hline \multicolumn{4}{|l|}{ Apodiformes } \\
\hline \multicolumn{4}{|l|}{ Apodidae } \\
\hline Cypseloides fumigatus (Streubel, 1848) & & & Fol \\
\hline Streptoprocne zonaris (Shaw, 1796) & * & & Rv/sP, Fol \\
\hline Chaetura meridionalis Hellmayr, 1907 & & INTRA & ExM, Rv/sP, Rv/sNP, Fol \\
\hline [Tachornis squamata (Cassin, 1853)] & & & $\mathrm{Rv} / \mathrm{sP}$ \\
\hline \multicolumn{4}{|l|}{ Trochilidae } \\
\hline Glaucis hirsutus (Gmelin, 1788) & * & & ExM, Rv/sP, Rv/sNP, Fol \\
\hline Phaethornis nattereri Berlepsch, 1887 & * & & ExM, Rv/sP, Fol \\
\hline [Phaethornis ruber (Linnaeus, 1758)] & * & & $\mathrm{Rv} / \mathrm{sP}$ \\
\hline Phaethornis subochraceus Todd, 1915 & ${ }^{*} \mathrm{DD}(\mathrm{BL})$ & & ExM, Rv/sP, Rv/sNP, Fol, ASI \\
\hline Phaethornis pretrei (Lesson \& Delattre, 1839) & & & ExM, Rv/sP, Rv/sNP, Fol \\
\hline Colibri serrirostris (Vieillot, 1816) & & & $\mathrm{Rv} / \mathrm{sP}, \mathrm{Fol}$ \\
\hline Heliactin bilophus (Temminck, 1820) & & & ExM, Rv/sP \\
\hline Polytmus guainumbi (Pallas, 1764) & & & ExM, Rv/sP, Rv/sNP, Fol, ASI \\
\hline Chrysolampis mosquitus (Linnaeus, 1758) & & & ExM, Rv/sP, Rv/sNP, FoP, Fol \\
\hline Anthracothorax nigricollis (Vieillot, 1817) & & INTRA & ExM, Rv/sP, Rv/sNP, Fol \\
\hline Lophornis gouldii (Lesson, 1832) & VU (GL) & & ExM, Rv/sP \\
\hline
\end{tabular}




\begin{tabular}{|c|c|c|c|}
\hline \multirow{2}{*}{ Taxon } & \multicolumn{2}{|c|}{ Status } & \multirow{2}{*}{ Evidence } \\
\hline & Conservation & Migration & \\
\hline Lophornis magnificus (Vieillot, 1817) & & & $\mathrm{Rv} / \mathrm{sP}, \mathrm{Fol}$ \\
\hline Heliomaster longirostris (Audebert \& Vieillot, 1801) & & & $\mathrm{Rv} / \mathrm{sP}, \mathrm{Fol}$ \\
\hline Heliomaster furcifer (Shaw, 1812) & * & & ExM, Rv/sP, Rv/sNP, Fol \\
\hline Calliphlox amethystina (Boddaert, 1783) & * & & ExM, Rv/sP, Rv/sNP, Fol \\
\hline Chlorostilbon lucidus (Shaw, 1812) & & & ExM, Rv/sP, Rv/sNP, Fol, ASI \\
\hline Thalurania furcata (Gmelin, 1788) & & & ExM, Rv/sP, Rv/sNP, Fol, ASI \\
\hline Eupetomena macroura (Gmelin, 1788) & & & ExM, Rv/sP, Rv/sNP, Fol \\
\hline Aphantochroa cirrochloris (Vieillot, 1818) & & & $\mathrm{Rv} / \mathrm{sP}, \mathrm{Fol}$ \\
\hline Chrysuronia versicolor (Vieillot, 1818) & & & ExM, Rv/sP, Rv/sNP \\
\hline Leucochloris albicollis (Vieillot, 1818) & & & Fol \\
\hline Chionomesa fimbriata (Gmelin, 1788) & & & ExM, Rv/sP, Rv/sNP, Fol, ASI \\
\hline [Hylocharis sapphirina (Gmelin, 1788)] & & & $\mathrm{Rv} / \mathrm{sP}, \mathrm{Rv} / \mathrm{sNP}$ \\
\hline Hylocharis chrysura (Shaw, 1812) & & & ExM, Rv/sP, Rv/sNP, Fol, ASI \\
\hline Chlorestes cyanus (Vieillot, 1818) & & & ExM, Rv/sP \\
\hline \multicolumn{4}{|l|}{ Gruiformes } \\
\hline \multicolumn{4}{|l|}{ Aramidae } \\
\hline Aramus guarauna (Linnaeus, 1766) & & INTRA & ExM, Rv/sP, Rv/sNP, Fol, ASI \\
\hline \multicolumn{4}{|l|}{ Rallidae } \\
\hline Porphyrio martinica (Linnaeus, 1766) & * & INTRA & ExM, Rv/sP, Rv/sNP, Fol \\
\hline Porphyrio flavirostris (Gmelin, 1789) & & & ExM, Rv/sP, Rv/sNP, Fol, ASI \\
\hline Rufirallus viridis (Statius Muller, 1776) & & & $\mathrm{Rv} / \mathrm{sP}, \mathrm{Fol}, \mathrm{ASI}$ \\
\hline Laterallus flaviventer (Boddaert, 1783) & $\mathrm{DD}(\mathrm{BL})$ & & Fol \\
\hline Laterallus melanophaius (Vieillot, 1819) & & & $\mathrm{Rv} / \mathrm{sP}, \mathrm{Rv} / \mathrm{sNP}$, Fol, ASI \\
\hline Laterallus exilis (Temminck, 1831) & & & ExM, Rv/sP, Rv/sNP, Fol, ASI \\
\hline [Laterallus xenopterus Conover, 1934] & ${ }^{*} \mathrm{VU}(\mathrm{GL}), \mathrm{DD}(\mathrm{BL})$ & & $\mathrm{Rv} / \mathrm{sNP}$ \\
\hline [Coturnicops notatus (Gould, 1841)] & ${ }^{*} \mathrm{DD}(\mathrm{BL})$ & & $\mathrm{Rv} / \mathrm{sP}$ \\
\hline Mustelirallus albicollis (Vieillot, 1819) & & & ExM, Rv/sP, Rv/sNP, Fol, ASI \\
\hline [Neocrex erythrops (Sclater, 1867)] & & INTRA & $\mathrm{Rv} / \mathrm{sP}, \mathrm{Rv} / \mathrm{sNP}$ \\
\hline Pardirallus maculatus (Boddaert, 1783) & & INTRA & $\mathrm{Rv} / \mathrm{sP}, \mathrm{FoP}, \mathrm{Fol}$ \\
\hline Pardirallus nigricans (Vieillot, 1819) & & & $\mathrm{Rv} / \mathrm{sP}, \mathrm{Rv} / \mathrm{sNP}, \mathrm{Fol}$ \\
\hline Aramides ypecaha (Vieillot, 1819) & * & & ExM, Rv/sP, Rv/sNP, Fol \\
\hline Aramides cajaneus (Statius Muller, 1776) & & & ExM, Rv/sP, Rv/sNP, Fol, ASI \\
\hline Gallinula galeata (Lichtenstein, 1918) & & INTRA & ExM, Rv/sP, Rv/sNP \\
\hline [Fulica leucoptera Vieillot, 1817] & & INTRA & $\mathrm{Rv} / \mathrm{sP}$ \\
\hline \multicolumn{4}{|l|}{ Heliornithidae } \\
\hline Heliornis fulica (Boddaert, 1783) & & & ExM, Rv/sP, Rv/sNP, Fol, ASI \\
\hline \multicolumn{4}{|l|}{ Charadriiformes } \\
\hline \multicolumn{4}{|l|}{ Charadriidae } \\
\hline Pluvialis dominica (Statius Muller, 1776) & ${ }^{*} \mathrm{DD}(\mathrm{BL})$ & INTER & ExM, Rv/sP, Rv/sNP, Fol \\
\hline [Pluvialis squatarola (Linnaeus, 1758)] & * & INTER & $\mathrm{Rv} / \mathrm{sP}$ \\
\hline Vanellus cayanus (Latham, 1790) & & INTRA & ExM, Rv/sP, Rv/sNP, Fol, ASI \\
\hline Vanellus chilensis (Molina, 1782) & & & ExM, Rv/sP, Rv/sNP, Fol, ASI \\
\hline [Charadrius semipalmatus Bonaparte, 1825] & & INTRA & $\mathrm{Rv} / \mathrm{sP}$ \\
\hline Charadrius collaris Vieillot, 1818 & * & INTRA & ExM, Rv/sP, Rv/sNP, Fol \\
\hline \multicolumn{4}{|l|}{ Recurvirostridae } \\
\hline Himantopus mexicanus (Statius Muller, 1776) & & INTRA & $\mathrm{Rv} / \mathrm{sP}, \mathrm{Fol}$ \\
\hline Himantopus melanurus Vieillot, 1817 & & INTRA & ExM, Rv/sP, Fol \\
\hline \multicolumn{4}{|l|}{ Scolopacidae } \\
\hline Bartramia longicauda (Bechstein, 1812) & & INTER & ExM, Rv/sP, Rv/sNP, Fol \\
\hline Numenius borealis (Forster, 1772) & $\mathrm{CR} / \mathrm{PE}(\mathrm{GL})$ & INTER & ExM, Rv/sP \\
\hline [Numenius hudsonicus Latham, 1790] & * & INTER & $\mathrm{Rv} / \mathrm{sP}$ \\
\hline Limosa haemastica (Linnaeus, 1758) & * & INTER & ExM, Rv/sP, Rv/sNP, Fol \\
\hline [Arenaria interpres (Linnaeus, 1758)] & * & INTER & $\mathrm{Rv} / \mathrm{sP}$ \\
\hline [Calidris canutus (Linnaeus, 1758)] & ${ }^{*} \mathrm{NT}(\mathrm{GL}), \mathrm{CR}(\mathrm{BL})$ & INTER & $\mathrm{Rv} / \mathrm{sP}$ \\
\hline Calidris himantopus (Bonaparte, 1826) & & INTER & $\mathrm{Rv} / \mathrm{sP}, \mathrm{Fol}$ \\
\hline Calidris alba (Pallas, 1764) & & INTER & $\mathrm{Rv} / \mathrm{sP}, \mathrm{Fol}$ \\
\hline Calidris bairdii (Coues, 1861) & & INTER & $\mathrm{Rv} / \mathrm{sP}, \mathrm{Fol}$ \\
\hline [Calidris minutilla (Vieillot, 1819)] & ${ }^{*} \mathrm{DD}(\mathrm{BL})$ & INTER & $\mathrm{Rv} / \mathrm{sP}$ \\
\hline Calidris fuscicollis (Vieillot, 1819) & * & INTER & ExM, Rv/sP, Rv/sNP, Fol, ASI \\
\hline Calidris subruficollis (Vieillot, 1819) & ${ }^{*} \mathrm{NT}(\mathrm{GL}), \mathrm{VU}(\mathrm{BL})$ & INTER & $\mathrm{Rv} / \mathrm{sP}$, Fol \\
\hline
\end{tabular}




\begin{tabular}{|c|c|c|c|}
\hline \multirow{2}{*}{ Taxon } & \multicolumn{2}{|c|}{ Status } & \multirow{2}{*}{ Evidence } \\
\hline & Conservation & Migration & \\
\hline Calidris melanotos (Vieillot, 1819) & & INTER & ExM, Rv/sP, Rv/sNP, FoP, Fol \\
\hline [Calidris pusilla (Linnaeus, 1766)] & ${ }^{*} \mathrm{NT}(\mathrm{GL}), \mathrm{EN}(\mathrm{BL})$ & INTER & $\mathrm{Rv} / \mathrm{sP}$ \\
\hline [Gallinago undulata (Boddaert, 1783)] & ${ }^{*} \mathrm{DD}(\mathrm{BL})$ & INTRA & $\mathrm{Rv} / \mathrm{sP}$ \\
\hline Gallinago paraguaiae (Vieillot, 1816) & & INTRA & ExM, Rv/sP, Rv/sNP, Fol \\
\hline Phalaropus tricolor (Vieillot, 1819) & $\mathrm{DD}(\mathrm{BL})$ & INTER & $\mathrm{ExM}, \mathrm{Rv} / \mathrm{sP}, \mathrm{Rv} / \mathrm{sNP}$, Fol \\
\hline Actitis macularius (Linnaeus, 1766) & * & INTER & ExM, Rv/sP, Rv/sNP, Fol, ASI \\
\hline Tringa solitaria Wilson, 1813 & & INTER & ExM, Rv/sP, Rv/sNP, Fol, ASI \\
\hline Tringa melanoleuca (Gmelin, 1789) & & INTER & ExM, Rv/sP, Rv/sNP, Fol, ASI \\
\hline Tringa flavipes (Gmelin, 1789) & * & INTER & ExM, Rv/sP, Rv/sNP, Fol, ASI \\
\hline \multicolumn{4}{|l|}{ Jacanidae } \\
\hline Jacana jacana (Linnaeus, 1766) & & & ExM, Rv/sP, Rv/sNP, Fol, ASI \\
\hline \multicolumn{4}{|l|}{ Laridae } \\
\hline Xema sabini (Sabine, 1819) & & INTER & Fol \\
\hline Chroicocephalus maculipennis (Lichtenstein, 1823) & & INTRA & Fol \\
\hline [Chroicocephalus cirrocephalus (Vieillot, 1818)] & & INTRA & $\mathrm{Rv} / \mathrm{sP}$ \\
\hline Leucophaeus pipixcan (Wagler, 1831) & & INTER & FoP \\
\hline Rynchops niger Linnaeus, 1758 & * & INTRA & ExM, Rv/sP, Rv/sNP, Fol, ASI \\
\hline Sternula superciliaris (Vieillot, 1819) & & INTRA & ExM, Rv/sP, Rv/sNP, Fol \\
\hline Phaetusa simplex (Gmelin, 1789) & & INTRA & ExM, Rv/sP, Rv/sNP, Fol, ASI \\
\hline Chlidonias niger (Linnaeus, 1758) & $*$ & INTER & $\mathrm{Rv} / \mathrm{sP}, \mathrm{FoP}$ \\
\hline [Sterna hirundo Linnaeus, 1758] & & INTER & $\mathrm{Rv} / \mathrm{sP}$ \\
\hline Sterna trudeaui Audubon, 1838 & & INTRA & $\mathrm{Rv} / \mathrm{sP}, \mathrm{Fol}$ \\
\hline \multicolumn{4}{|l|}{ Eurypygiformes } \\
\hline \multicolumn{4}{|l|}{ Eurypygidae } \\
\hline Eurypyga helias (Pallas, 1781) & * & & ExM, Rv/sP, Rv/sNP, Fol, ASI \\
\hline \multicolumn{4}{|l|}{ Ciconiiformes } \\
\hline \multicolumn{4}{|l|}{ Ciconiidae } \\
\hline Ciconia maguari (Gmelin, 1789) & & INTRA & ExM, Rv/sP, Rv/sNP, Fol \\
\hline Jabiru mycteria (Lichtenstein, 1819) & & INTRA & ExM, Rv/sP, Rv/sNP, Fol, ASI \\
\hline Mycteria americana Linnaeus, 1758 & * & INTRA & ExM, Rv/sP, Rv/sNP, Fol \\
\hline \multicolumn{4}{|l|}{ Suliformes } \\
\hline \multicolumn{4}{|l|}{ Anhingidae } \\
\hline Anhinga anhinga (Linnaeus, 1766) & * & & ExM, Rv/sP, Rv/sNP, Fol, ASI \\
\hline \multicolumn{4}{|l|}{ Phalacrocoracidae } \\
\hline Nannopterum brasilianus (Gmelin, 1789) & & INTRA & ExM, Rv/sP, Rv/sNP, Fol, ASI \\
\hline \multicolumn{4}{|l|}{ Pelecaniformes } \\
\hline \multicolumn{4}{|l|}{ Ardeidae } \\
\hline Tigrisoma lineatum (Boddaert, 1783) & & & ExM, Rv/sP, Rv/sNP, Fol, ASI \\
\hline Agamia agami (Gmelin, 1789) & * VU (GL) & & ExM, Rv/sP, FoP, Fol, ASI \\
\hline Cochlearius cochlearius (Linnaeus, 1766) & * & & ExM, Rv/sP, Rv/sNP, Fol, ASI \\
\hline Zebrilus undulatus (Gmelin, 1789) & ${ }^{*} \mathrm{NT}(\mathrm{GL})$ & & ExM, Rv/sP, FoP, Fol, ASI \\
\hline Botaurus pinnatus (Wagler, 1829) & & & $\mathrm{Rv} / \mathrm{sP}, \mathrm{Fol}$ \\
\hline Ixobrychus exilis (Gmelin, 1789) & & & ExM, Rv/sP, Rv/sNP, Fol, ASI \\
\hline Ixobrychus involucris (Vieillot, 1823) & & & $\mathrm{Rv} / \mathrm{sP}, \mathrm{Fol}$ \\
\hline Nycticorax nycticorax (Linnaeus, 1758) & * & & ExM, Rv/sP, Rv/sNP, Fol, ASI \\
\hline Butorides striata (Linnaeus, 1758) & * & & ExM, Rv/sP, Rv/sNP, Fol, ASI \\
\hline Bubulcus ibis (Linnaeus, 1758) & & & $\mathrm{Rv} / \mathrm{sP}, \mathrm{Rv} / \mathrm{sNP}, \mathrm{Fol}$ \\
\hline Ardea cocoi Linnaeus, 1766 & & & ExM, Rv/sP, Rv/sNP, Fol, ASI \\
\hline Ardea alba Linnaeus, 1758 & & INTRA & ExM, Rv/sP, Rv/sNP, Fol, ASI \\
\hline Syrigma sibilatrix (Temminck, 1824) & & & ExM, Rv/sP, Rv/sNP, Fol \\
\hline Pilherodius pileatus (Boddaert, 1783) & & & ExM, Rv/sP, Rv/sNP, Fol \\
\hline Egretta thula (Molina, 1782) & & & ExM, Rv/sP, Rv/sNP, Fol, ASI \\
\hline Egretta caerulea (Linnaeus, 1758) & & INTRA & ExM, Rv/sP, Rv/sNP, FoP, Fol \\
\hline \multicolumn{4}{|l|}{ Threskiornithidae } \\
\hline Plegadis chihi (Vieillot, 1817) & & INTRA & ExM, Rv/sP, Rv/sNP, Fol, ASI \\
\hline Mesembrinibis cayennensis (Gmelin, 1789) & * & & ExM, Rv/sP, Rv/sNP, Fol, ASI \\
\hline Phimosus infuscatus (Lichtenstein, 1823) & & INTRA & ExM, Rv/sP, Rv/sNP, Fol, ASI \\
\hline Theristicus caerulescens (Vieillot, 1817) & & & ExM, Rv/sP, Rv/sNP, Fol, ASI \\
\hline Theristicus caudatus (Boddaert, 1783) & & & ExM, Rv/sP, Rv/sNP, Fol, ASI \\
\hline Platalea ajaja Linnaeus, 1758 & & INTRA & ExM, Rv/sP, Rv/sNP, Fol, ASI \\
\hline
\end{tabular}




\section{Cathartiformes}

\section{Cathartidae}

Sarcoramphus papa (Linnaeus, 1758)

Coragyps atratus (Bechstein, 1793)

Cathartes burrovianus Cassin, 1845

\section{Accipitriformes}

\section{Pandionidae}

Pandion haliaetus (Linnaeus, 1758)

\section{Accipitridae}

Gampsonyx swainsonii Vigors, 1825

Elanus leucurus (Vieillot, 1818)

Chondrohierax uncinatus (Temminck, 1822)

Leptodon cayanensis (Latham, 1790)

Elanoides forficatus (Linnaeus, 1758)

Harpia harpyja (Linnaeus, 1758)

Spizaetus tyrannus (Wied, 1820)

Spizaetus melanoleucus (Vieillot, 1816)

Spizaetus ornatus (Daudin, 1800)

Busarellus nigricollis (Latham, 1790)

Rostrhamus sociabilis (Vieillot, 1817)

Helicolestes hamatus (Temminck, 1821)

Harpagus bidentatus (Latham, 1790)

Harpagus diodon (Temminck, 1823)

Ictinia mississippiensis (Wilson, 1811)

Ictinia plumbea (Gmelin, 1788)

Circus buffoni (Gmelin, 1788)

Hieraspiza superciliosa (Linnaeus, 1766)

Accipiter poliogaster (Temminck, 1824)

Accipiter striatus Vieillot, 1808

Accipiter bicolor (Vieillot, 1817)

Geranospiza caerulescens (Vieillot, 1817)

Heterospizias meridionalis (Latham, 1790)

Urubitinga urubitinga (Gmelin, 1788)

Urubitinga coronata (Vieillot, 1817)

Rupornis magnirostris (Gmelin, 1788)

Parabuteo unicinctus (Temminck, 1824)

Geranoaetus albicaudatus (Vieillot, 1816)

Geranoaetus melanoleucus (Vieillot, 1819)

Pseudastur albicollis (Latham, 1790)

Buteo nitidus (Latham, 1790)

[Buteo platypterus (Vieillot, 1823)]

Buteo brachyurus Vieillot, 1816

Buteo swainsoni Bonaparte, 1838

Buteo albonotatus Kaup, 1847

\section{Strigiformes}

\section{Tytonidae}

Tyto furcata (Temminck, 1827)

\section{Strigidae}

Megascops choliba (Vieillot, 1817)

Megascops usta (Sclater, 1858)

Pulsatrix perspicillata (Latham, 1790)

Bubo virginianus (Gmelin, 1788)

Strix virgata (Cassin, 1849)

Strix huhula Daudin, 1800

Glaucidium hardyi Vielliard, 1990

Glaucidium brasilianum (Gmelin, 1788)

Athene cunicularia (Molina, 1782)

[Aegolius harrisii (Cassin, 1849)]

Asio clamator (Vieillot, 1808)
Cathartes aura (Linnaeus, 1758)
Evidence

${ }^{*} \mathrm{NT}(\mathrm{BL})$

\section{Status}

Conservation

Migration

EM, Rv/sP, Rv/sNP, Fol

ExM, Rv/sP, Rv/sNP, Fol

ExM, Rv/sP, Rv/sNP, Fol

Rv/sP, Rv/sNP, Fol

INTER

ExM, Rv/sP, Rv/sNP, Fol

ExM, Rv/sP, Rv/sNP, Fol

INTRA

ExM, Rv/sP, Rv/sNP,

ExM, Rv/sP, Rv/sNP, Fol

ExM, Rv/sP, Rv/sNP, Fol

${ }^{*} \mathrm{NT}(\mathrm{GL}), \mathrm{VU}(\mathrm{BL})$

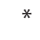

${ }^{*} \mathrm{NT}(\mathrm{GL}), \mathrm{NT}(\mathrm{BL})$

*

INTRA

INTER

INTRA

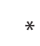

${ }^{*} \mathrm{NT}(\mathrm{GL}), \mathrm{DD}(\mathrm{BL})$

${ }^{*} \mathrm{EN}(\mathrm{GL}), \mathrm{EN}(\mathrm{BL})$

INTER

INTRA

INTER

Rv/sP, FoP, Fol

ExM, Rv/sP, FoP, Fol

$\mathrm{Rv} / \mathrm{sP}, \mathrm{Fol}$

ExM, Rv/sP, Rv/sNP, Fol, ASI

ExM, Rv/sP, Rv/sNP, Fol, ASI

$\mathrm{Rv} / \mathrm{sP}, \mathrm{Fol}$

Fol

$\mathrm{Rv} / \mathrm{sP}, \mathrm{Rv} / \mathrm{sNP}, \mathrm{Fol}$

$\mathrm{Rv} / \mathrm{sP}, \mathrm{Rv} / \mathrm{sNP}, \mathrm{Fol}$

ExM, Rv/sP, Rv/sNP, Fol, ASI

ExM, Rv/sP, Rv/sNP, Fol, ASI

$\mathrm{Rv} / \mathrm{sP}, \mathrm{Rv} / \mathrm{sNP}, \mathrm{Fol}$

Rv/sP, FoP, Fol

$\mathrm{Rv} / \mathrm{sP}, \mathrm{Rv} / \mathrm{sNP}, \mathrm{Fol}$

ExM, Rv/sP, Rv/sNP, Fol

ExM, Rv/sP, Rv/sNP, Fol, ASI

ExM, Rv/sP, Rv/sNP, Fol

ExM, Rv/sP, Rv/sNP, Fol, ASI

$\mathrm{Rv} / \mathrm{sP}, \mathrm{Rv} / \mathrm{sNP}$, FoP, Fol, ASI

ExM, Rv/sP, Rv/sNP, Fol, ASI

$\mathrm{Rv} / \mathrm{sP}, \mathrm{FoP}$, Fol

ExM, Rv/sP, Rv/sNP, Fol

Rv/sP, FoP, Fol

ExM, Rv/sP, Rv/sNP, Fol

$\mathrm{Rv} / \mathrm{sP}$

ExM, Rv/sP, Rv/sNP

Fol
ExM, Rv/sP, Rv/sNP, Fol, ASI

ExM, Rv/sP, Rv/sNP, Fol

ExM, Rv/sP, Rv/sNP, Fol, FOAP

$\mathrm{Rv} / \mathrm{sP}, \mathrm{Rv} / \mathrm{sNP}$, Fol

ExM, Rv/sP, Rv/sNP, Fol

ExM, Rv/sP, Rv/sNP, Fol, ASI

Fol, ASI

ExM, Rv/sP, Rv/sNP, Fol

ExM, Rv/sP, Rv/sNP, Fol, ASI

Rv/sP, Fol, ASI

ExM, Rv/sP, Rv/sNP, Fol, ASI

ExM, Rv/sP

ExM, Rv/sP, Rv/sNP, Fol, ASI

ExM, Rv/sP, Rv/sNP, Fol

$\mathrm{Rv} / \mathrm{sP}$

ExM, Rv/sP, Rv/sNP, 


\begin{tabular}{|c|c|c|c|}
\hline \multirow{2}{*}{ Taxon } & \multicolumn{2}{|c|}{ Status } & \multirow{2}{*}{ Evidence } \\
\hline & Conservation & Migration & \\
\hline Asio stygius (Wagler, 1832) & * & & ExM, Rv/sP, Rv/sNP, ASI \\
\hline Asio flammeus (Pontoppidan, 1763) & $*$ & & $\mathrm{Rv} / \mathrm{sP}, \mathrm{Fol}$ \\
\hline \multicolumn{4}{|l|}{ Trogoniformes } \\
\hline \multicolumn{4}{|l|}{ Trogonidae } \\
\hline Trogon melanurus Swainson, 1838 & & & ExM, Rv/sP \\
\hline Trogon curucui Linnaeus, 1766 & * & & ExM, Rv/sP, Rv/sNP, Fol, ASI \\
\hline \multicolumn{4}{|l|}{ Coraciiformes } \\
\hline \multicolumn{4}{|l|}{ Momotidae } \\
\hline Momotus momota (Linnaeus, 1766) & * & & ExM, Rv/sP, Rv/sNP, Fol, ASI \\
\hline \multicolumn{4}{|l|}{ Alcedinidae } \\
\hline Megaceryle torquata (Linnaeus, 1766) & & & ExM, Rv/sP, Rv/sNP, Fol, ASI \\
\hline Chloroceryle amazona (Latham, 1790) & & & ExM, Rv/sP, Rv/sNP, Fol, ASI \\
\hline Chloroceryle aenea (Pallas, 1764) & * & & ExM, Rv/sP, Rv/sNP, Fol, ASI \\
\hline Chloroceryle americana (Gmelin, 1788) & & & ExM, Rv/sP, Rv/sNP, Fol, ASI \\
\hline Chloroceryle inda (Linnaeus, 1766) & * & & ExM, Rv/sP, Rv/sNP, Fol, ASI \\
\hline \multicolumn{4}{|l|}{ Galbuliformes } \\
\hline \multicolumn{4}{|l|}{ Galbulidae } \\
\hline Brachygalba lugubris (Swainson, 1838) & $*$ & & ExM, Rv/sP \\
\hline Galbula ruficauda Cuvier, 1816 & * & & ExM, Rv/sP, Rv/sNP, Fol, ASI \\
\hline \multicolumn{4}{|l|}{ Bucconidae } \\
\hline Chelidoptera tenebrosa (Pallas, 1782) & & & $\mathrm{Rv} / \mathrm{sP}, \mathrm{Rv} / \mathrm{sNP}, \mathrm{Fol}$ \\
\hline Monasa nigrifrons (Spix, 1824) & * & & ExM, Rv/sP, Rv/sNP, Fol, ASI \\
\hline Nonnula ruficapilla (Tschudi, 1844) & & & ExM \\
\hline [Nonnula rubecula (Spix, 1824)] & * & & $\mathrm{Rv} / \mathrm{sP}$ \\
\hline Nystalus maculatus (Gmelin, 1788) & & & ExM, Rv/sP, Fol \\
\hline Nystalus striatipectus (Sclater, 1854) & & & ExM, Rv/sP, Rv/sNP, Fol, ASI \\
\hline Nystalus chacuru (Vieillot, 1816) & & & ExM, Rv/sP, Rv/sNP, Fol \\
\hline \multicolumn{4}{|l|}{ Piciformes } \\
\hline \multicolumn{4}{|l|}{ Ramphastidae } \\
\hline Ramphastos toco Statius Muller, 1776 & $*$ & & ExM, Rv/sP, Rv/sNP, Fol, ASI \\
\hline Ramphastos vitellinus Lichtenstein, 1823 & * VU (GL) & & ExM, Rv/sP \\
\hline Pteroglossus inscriptus Swainson, 1822 & * & & Fol \\
\hline Pteroglossus castanotis Gould, 1834 & * & & ExM, Rv/sP, Rv/sNP, Fol, ASI \\
\hline Pteroglossus bitorquatus Vigors, 1826 & ${ }^{*}$ EN (GL), NT (BL) & & Fol \\
\hline \multicolumn{4}{|l|}{ Picidae } \\
\hline Picumnus aurifrons Pelzeln, 1870 & * & & ExM \\
\hline Picumnus cirratus Temminck, 1825 & * & & ExM, Rv/sP, Fol \\
\hline Picumnus albosquamatus d'Orbigny, 1840 & $*$ & & ExM, Rv/sP, Rv/sNP, Fol, ASI \\
\hline Melanerpes candidus (0tto, 1796) & & & ExM, Rv/sP, Rv/sNP, Fol, ASI \\
\hline Melanerpes cruentatus (Boddaert, 1783) & & & ExM, Rv/sP, Rv/sNP, Fol, ASI \\
\hline Melanerpes flavifrons (Vieillot, 1818) & & & $\mathrm{Rv} / \mathrm{sP}, \mathrm{Fol}$ \\
\hline Melanerpes cactorum (d'0rbigny, 1839) & * & & ExM, Rv/sP, Rv/sNP, Fol, ASI \\
\hline Veniliornis affinis (Swainson, 1821) & & & ExM, Rv/sNP, Fol \\
\hline Veniliornis passerinus (Linnaeus, 1766) & & & ExM, Rv/sP, Rv/sNP, Fol, ASI \\
\hline Veniliornis mixtus (Boddaert, 1783) & & & ExM, Rv/sP, Rv/sNP, Fol \\
\hline Campephilus rubricollis (Boddaert, 1783) & * & & Rv/sP, Fol, ASI \\
\hline Campephilus melanoleucos (Gmelin, 1788) & * & & ExM, Rv/sP, Rv/sNP, Fol, ASI \\
\hline Campephilus leucopogon (Valenciennes, 1826) & & & ExM, Rv/sP, Rv/sNP, Fol \\
\hline Dryocopus lineatus (Linnaeus, 1766) & & & ExM, Rv/sP, Rv/sNP, Fol, ASI \\
\hline Celeus torquatus (Boddaert, 1783) & & & ExM, Rv/sP \\
\hline Celeus flavus (Statius Muller, 1776) & ${ }^{*} \mathrm{NT}(\mathrm{BL})$ & & Rv/sP, Rv/sNP, FoP, Fol, ASI \\
\hline Celeus lugubris (Malherbe, 1851) & * & & ExM, Rv/sP, Rv/sNP, Fol, ASI \\
\hline Celeus flavescens (Gmelin, 1788) & * & & ExM, Rv/sP, Rv/sNP, \\
\hline Piculus chrysochloros (Vieillot, 1818) & & & ExM, Rv/sP, Rv/sNP, Fol, ASI \\
\hline Colaptes melanochloros (Gmelin, 1788) & & & ExM, Rv/sP, Rv/sNP, Fol, ASI \\
\hline Colaptes campestris (Vieillot, 1818) & & & ExM, Rv/sP, Rv/sNP, Fol, ASI \\
\hline \multicolumn{4}{|l|}{ Cariamiformes } \\
\hline \multicolumn{4}{|l|}{ Cariamidae } \\
\hline Cariama cristata (Linnaeus, 1766) & & & ExM, Rv/sP, Rv/sNP, Fol, ASI \\
\hline
\end{tabular}




\begin{tabular}{lccc}
\hline \multirow{2}{*}{ Taxon } & \multicolumn{2}{c}{ Status } & Migration \\
\cline { 3 - 4 } & Conservation & Evidence \\
\hline Falconiformes
\end{tabular}

\section{Falconiformes}

\section{Falconidae}

Herpetotheres cachinnans (Linnaeus, 1758)

Micrastur ruficollis (Vieillot, 1817)

Micrastur semitorquatus (Vieillot, 1817)

Caracara plancus (Miller, 1777)

Milvago chimachima (Vieillot, 1816)

Milvago chimango (Vieillot, 1816)

Falco sparverius Linnaeus, 1758

Falco rufigularis Daudin, 1800

Falco deiroleucus Temminck, 1825

Falco femoralis Temminck, 1822

Falco peregrinus Tunstall, 1771

\section{Psittaciformes}

\section{Psittacidae}

Myiopsitta monachus (Boddaert, 1783)

Brotogeris chiriri (Vieillot, 1818)

Pionus maximiliani (Kuhl, 1820)

Pionus menstruus (Linnaeus, 1766)

Alipiopsitta xanthops (Spix, 1824)

Amazona aestiva (Linnaeus, 1758)

Amazona amazonica (Linnaeus, 1766)

Forpus xanthopterygius (Spix, 1824)

Pyrrhura devillei (Massena \& Souancé, 1854)

Pyrrhura frontalis (Vieillot, 1817)

Pyrrhura molinae (Massena \& Souancé, 1854)

Anodorhynchus hyacinthinus (Latham, 1790)

Eupsittula aurea (Gmelin, 1788)

Aratinga nenday (Vieillot, 1823)

Orthopsittaca manilatus (Boddaert, 1783)

Primolius maracana (Vieillot, 1816)

Primolius auricollis (Cassin, 1853)

Ara ararauna (Linnaeus, 1758)

Ara chloropterus Gray, 1859

Thectocercus acuticaudatus (Vieillot, 1818)

Diopsittaca nobilis (Linnaeus, 1758)

Psittacara leucophthalmus (Statius Muller, 1776)

\section{Passeriformes}

\section{Thamnophilidae}

Myrmorchilus strigilatus (Wied, 1831)

[Formicivora grisea (Boddaert, 1783)]

Formicivora melanogaster Pelzeln, 1868

Formicivora rufa (Wied, 1831)

Dysithamnus mentalis (Temminck, 1823)

Herpsilochmus longirostris Pelzeln, 1868

Herpsilochmus atricapillus Pelzeln, 1868

Thamnophilus doliatus (Linnaeus, 1764)

Thamnophilus sticturus Pelzeln, 1868

Thamnophilus pelzelni Hellmayr, 1924

Thamnophilus caerulescens Vieillot, 1816

${ }^{*} \mathrm{NT}(\mathrm{GL}), \mathrm{NT}(\mathrm{BL})$

${ }^{*} \mathrm{NT}(\mathrm{GL}), \mathrm{NT}(\mathrm{BL})$

${ }^{*} \mathrm{NT}(\mathrm{GL}), \mathrm{NT}(\mathrm{BL})$

NT (GL), NT (BL)

${ }^{*} \mathrm{NT}(\mathrm{BL})$

* VU (GL), NT (BL)

* NT (BL)

[Thamnophilus amazonicus Sclater, 1858]

Taraba major (Vieillot, 1816)

Hypocnemoides maculicauda (Pelzeln, 1868)

Pyriglena maura (Ménétries, 1835)

Cercomacra cinerascens (Sclater, 1857)

Cercomacra melanaria (Ménétriès, 1835)

Drymophyla devillei (Ménégaux \& Hellmayr, 1906)

\section{Conopophagidae}

Conopophaga lineata (Wied, 1831)

Dendrocolaptidae

Sittasomus griseicapillus (Vieillot, 1818)

ExM, Rv/sP, Rv/sNP, Fol, ASI

ExM, Rv/sP

ExM, Rv/sP, Rv/sNP, Fol, ASI

ExM, Rv/sP, Rv/sNP, Fol, ASI

ExM, Rv/sP, Rv/sNP, Fol, ASI

Rv/sP, ASI

ExM, Rv/sP, Rv/sNP, Fol

ExM, Rv/sP, Rv/sNP, Fol, ASI

Rv/sP, Fol

ExM, Rv/sP, Rv/sNP, Fol

ExM, Rv/sP, Fol

ExM, Rv/sP, Rv/sNP, Fol, ASI

ExM, Rv/sP, Rv/sNP, Fol, ASI

ExM, Rv/sP, Rv/sNP, Fol, ASI

ExM, Rv/sP, Rv/sNP, Fol, ASI

ExM, Rv/sP, Rv/sNP, Fol, ASI

ExM, Rv/sP, Rv/sNP, Fol, ASI

ExM, Rv/sP, Rv/sNP, Fol, ASI

$\mathrm{Rv} / \mathrm{sP}, \mathrm{Rv} / \mathrm{sNP}, \mathrm{Fol}$

ExM, Rv/sP, Rv/sNP, ASI

Fol

ExM, Rv/sP, Rv/sNP, Fol

ExM, Rv/sP, Rv/sNP, Fol, ASI

ExM, Rv/sP, Rv/sNP, Fol, ASI

ExM, Rv/sP, Rv/sNP, Fol, ASI

ExM, Rv/sP, Fol

ExM, Rv/sP, Fol

ExM, Rv/sP, Rv/sNP, Fol, ASI

ExM, Rv/sP, Rv/sNP, Fol, ASI

ExM, Rv/sP, Rv/sNP, Fol, ASI

ExM, Rv/sP, Rv/sNP, Fol, ASI

ExM, Rv/sP, Rv/sNP, Fol, ASI

ExM, Rv/sP, Rv/sNP, Fol, ASI

ExM, Rv/sP, Rv/sNP

$\mathrm{Rv} / \mathrm{sP}$

ExM, Rv/sP, Fol, ASI

ExM, Rv/sP, Rv/sNP, Fol, ASI

ExM, Rv/sP, Rv/sNP, Fol, ASI

ExM, Rv/sP, Rv/sNP, Fol, ASI

$\mathrm{Rv} / \mathrm{sP}, \mathrm{Fol}$

ExM, Rv/sP, Rv/sNP, Fol, ASI

ExM, Rv/sP, Rv/sNP, Fol, ASI

ExM, Fol, ASI

ExM, Rv/sP, Rv/sNP, ASI

Rv/sP

ExM, Rv/sP, Rv/sNP, Fol, ASI

ExM, Rv/sP, Rv/sNP, Fol, ASI

ExM, Rv/sP, Rv/sNP, Fol, ASI

ExM, Rv/sP

ExM, Rv/sP, Rv/sNP, Fol, ASI

ExM, Rv/sP

ExM, Rv/sP, Fol 


\begin{tabular}{|c|c|c|c|}
\hline \multirow{2}{*}{ Taxon } & \multicolumn{2}{|c|}{ Status } & \multirow{2}{*}{ Evidence } \\
\hline & Conservation & Migration & \\
\hline Dendrocincla fuliginosa (Vieillot, 1818) & * & & $\mathrm{Rv} / \mathrm{sP}, \mathrm{Fol}$ \\
\hline [Glyphorynchus spirurus (Vieillot, 1819)] & * & & $\mathrm{Rv} / \mathrm{sP}$ \\
\hline Dendrocolaptes picumnus Lichtenstein, 1820 & & & ExM, Rv/sP, Rv/sNP, Fol, ASI \\
\hline Dendrocolaptes platyrostris Spix, 1825 & & & ExM, Rv/sP, Rv/sNP, Fol, ASI \\
\hline Xiphocolaptes major (Vieillot, 1818) & * & & ExM, Rv/sP, Rv/sNP, Fol, ASI \\
\hline Xiphorhynchus guttatoides (Lafresnaye, 1850) & * & & ExM, Rv/sP, Rv/sNP, Fol, ASI \\
\hline Dendroplex picus (Gmelin, 1788) & & & ExM, Rv/sP, Rv/sNP, Fol, ASI \\
\hline Campylorhamphus trochilirostris (Lichtenstein, 1820) & & & ExM, Rv/sP, Rv/sNP, Fol, ASI \\
\hline Lepidocolaptes angustirostris (Vieillot, 1818) & & & ExM, Rv/sP, Rv/sNP, Fol, ASI \\
\hline [Lepidocolaptes fuscicapillus (Pelzeln, 1868)] & & & $\mathrm{Rv} / \mathrm{sP}$ \\
\hline \multicolumn{4}{|l|}{ Xenopidae } \\
\hline Xenops rutilans Temminck, 1821 & & & ExM, Rv/sP, Rv/sNP, Fol \\
\hline \multicolumn{4}{|l|}{ Furnariidae } \\
\hline Furnarius leucopus Swainson, 1838 & & & ExM, Rv/sP, Rv/sNP, Fol, ASI \\
\hline Furnarius rufus (Gmelin, 1788) & & & ExM, Rv/sP, Rv/sNP, Fol, ASI \\
\hline Phleocryptes melanops (Vieillot, 1817) & * & & Fol \\
\hline Dendroma rufa (Vieillot, 1818) & * & & $\mathrm{Rv} / \mathrm{sP}, \mathrm{Fol}$ \\
\hline Clibanornis rectirostris (Wied, 1831) & * & & ExM, Rv/sP, Fol, ASI \\
\hline Phacellodomus rufifrons (Wied, 1821) & & & ExM, Rv/sP, Rv/sNP, Fol, ASI \\
\hline Phacellodomus ruber (Vieillot, 1817) & * & & ExM, Rv/sP, Rv/sNP, Fol, ASI \\
\hline Anumbius annumbi (Vieillot, 1817) & & & ExM, Rv/sP, Fol \\
\hline Cranioleuca vulpina (Pelzeln, 1856) & & & ExM, Rv/sP, Rv/sNP, Fol, ASI \\
\hline Cranioleuca pyrrhophia (Vieillot, 1818) & & & Fol \\
\hline Pseudoseisura unirufa (d'Orbigny \& Lafresnaye, 1838) & & & ExM, Rv/sP, Rv/sNP, Fol, ASI \\
\hline Certhiaxis cinnamomeus (Gmelin, 1788) & * & & ExM, Rv/sP, Rv/sNP, Fol, ASI \\
\hline Schoeniophylax phryganophilus (Vieillot, 1817) & & & ExM, Rv/sP, Rv/sNP, Fol, ASI \\
\hline Synallaxis scutata Sclater, 1859 & * & & ExM, Rv/sP \\
\hline Synallaxis albilora Pelzeln, 1856 & & & ExM, Rv/sP, Rv/sNP, Fol, ASI \\
\hline Synallaxis hypospodia Sclater, 1874 & & & ExM, Rv/sP, Rv/sNP, Fol, ASI \\
\hline Synallaxis albescens Temminck, 1823 & & & ExM, Rv/sP, Rv/sNP \\
\hline Synallaxis frontalis Pelzeln, 1859 & & & ExM, Rv/sP, Rv/sNP, Fol, ASI \\
\hline \multicolumn{4}{|l|}{ Pipridae } \\
\hline Neopelma pallescens (Lafresnaye, 1853) & & & ExM, Rv/sP, Rv/sNP, Fol, ASI \\
\hline [Chiroxiphia caudata (Shaw \& Nodder, 1793)] & & & $\mathrm{Rv} / \mathrm{sP}$ \\
\hline Antilophia galeata (Lichtenstein, 1823) & * & & ExM, Rv/sP, Rv/sNP, Fol, ASI \\
\hline [Manacus manacus (Linnaeus, 1766)] & & & $\mathrm{Rv} / \mathrm{sP}$ \\
\hline Pipra fasciicauda Hellmayr, 1906 & & & ExM, Rv/sP, Rv/sNP, Fol, ASI \\
\hline Machaeropterus pyrocephalus (Sclater, 1852) & * & & Fol, ASI \\
\hline \multicolumn{4}{|l|}{ Cotingidae } \\
\hline Cephalopterus ornatus Saint-Hilaire, 1809 & & & ExM, Rv/sP \\
\hline Gymnoderus foetidus (Linnaeus, 1758) & * & & ExM, Rv/sP, Fol \\
\hline \multicolumn{4}{|l|}{ Tityridae } \\
\hline Tityra inquisitor (Lichtenstein, 1823) & & & ExM, Rv/sP, Rv/sNP, Fol, ASI \\
\hline Tityra cayana (Linnaeus, 1766) & * & INTRA & ExM, Rv/sP, Rv/sNP, Fol \\
\hline Tityra semifasciata (Spix, 1825) & & & ExM, Rv/sP, Fol \\
\hline Pachyramphus viridis (Vieillot, 1816) & & INTRA & ExM, Rv/sP, Rv/sNP, Fol, ASI \\
\hline [Pachyramphus castaneus (Jardine \& Selby, 1827)] & & & $\mathrm{Rv} / \mathrm{sP}$ \\
\hline Pachyramphus polychopterus (Vieillot, 1818) & & INTRA & ExM, Rv/sP, Rv/sNP, Fol, ASI \\
\hline Pachyramphus marginatus (Lichtenstein, 1823) & * & & $\mathrm{Rv} / \mathrm{sP}, \mathrm{Fol}$ \\
\hline Pachyramphus validus (Lichtenstein, 1823) & * & INTRA & $\mathrm{Rv} / \mathrm{sP}, \mathrm{Fol}$ \\
\hline Xenopsaris albinucha (Burmeister, 1869) & & & ExM, Rv/sP, Rv/sNP, Fol \\
\hline \multicolumn{4}{|l|}{ Onychorhynchidae } \\
\hline [Myiobius barbatus (Gmelin, 1789)] & & & $\mathrm{Rv} / \mathrm{sP}$ \\
\hline \multicolumn{4}{|l|}{ Platyrinchidae } \\
\hline Platyrinchus mystaceus Vieillot, 1818 & & & $\mathrm{Rv} / \mathrm{sP}, \mathrm{Fol}$ \\
\hline \multicolumn{4}{|l|}{ Rhynchocyclidae } \\
\hline Mionectes oleagineus (Lichtenstein, 1823) & & & $\mathrm{Rv} / \mathrm{sP}, \mathrm{Fol}$ \\
\hline Leptopogon amaurocephalus Tschudi, 1846 & * & & ExM, Rv/sP, Rv/sNP, ASI \\
\hline Corythopis delalandi (Lesson, 1830) & & & ExM, Rv/sP, ASI \\
\hline Tolmomyias sulphurescens (Spix, 1825) & & & ExM, Rv/sP, Rv/sNP, Fol, ASI \\
\hline
\end{tabular}




\begin{tabular}{|c|c|c|c|}
\hline \multirow{2}{*}{ Taxon } & \multicolumn{2}{|c|}{ Status } & \multirow{2}{*}{ Evidence } \\
\hline & Conservation & Migration & \\
\hline Tolmomyias flaviventris (Wied, 1831) & & & $\mathrm{Rv} / \mathrm{sP}, \mathrm{Fol}, \mathrm{ASI}$ \\
\hline Todirostrum cinereum (Linnaeus, 1766) & & & ExM, Rv/sP, Rv/sNP, Fol, ASI \\
\hline Poecilotriccus latirostris (Pelzeln, 1868) & & & ExM, Rv/sP, Rv/sNP, Fol, ASI \\
\hline [Myiornis ecaudatus (d'Orbigny \& Lafresnaye, 1837)] & * & & $\mathrm{Rv} / \mathrm{sP}$ \\
\hline Hemitriccus striaticollis (Lafresnaye, 1853) & * & & ExM, Rv/sP, Fol, ASI \\
\hline Hemitriccus margaritaceiventer (d'Orbigny \& Lafresnaye, 1837) & & & ExM, Rv/sP, Rv/sNP, Fol, ASI \\
\hline \multicolumn{4}{|l|}{ Tyrannidae } \\
\hline Hirundinea ferruginea (Gmelin, 1788) & & INTRA & $\mathrm{Rv} / \mathrm{sP}, \mathrm{Fol}$ \\
\hline Inezia inornata (Salvadori, 1897) & * & INTRA & ExM, Rv/sP, Rv/sNP, Fol, ASI \\
\hline Euscarthmus meloryphus Wied, 1831 & & & ExM, Rv/sP, Rv/sNP, Fol, ASI \\
\hline Camptostoma obsoletum (Temminck, 1824) & & & ExM, Rv/sP, Rv/sNP, Fol, ASI \\
\hline Elaenia flavogaster (Thunberg, 1822) & & & ExM, Rv/sP, Rv/sNP, Fol, ASI \\
\hline Elaenia spectabilis Pelzeln, 1868 & * & INTRA & ExM, Rv/sP, Rv/sNP, Fol, ASI \\
\hline Elaenia chilensis Hellmayr, 1927 & & INTRA & ExM, Rv/sP, FoP \\
\hline Elaenia parvirostris Pelzeln, 1868 & & INTRA & ExM, Rv/sP, Fol \\
\hline [Elaenia mesoleuca (Deppe, 1830)] & * & INTRA & $\mathrm{Rv} / \mathrm{sP}$ \\
\hline [Elaenia cristata Pelzeln, 1868] & * & INTRA & $\mathrm{Rv} / \mathrm{sP}, \mathrm{Rv} / \mathrm{sNP}$ \\
\hline Elaenia chiriquensis Lawrence, 1865 & * & INTRA & ExM, Rv/sP \\
\hline [Elaenia obscura (d'Orbigny \& Lafresnaye, 1837)] & & & $\mathrm{Rv} / \mathrm{sP}$ \\
\hline Suiriri suiriri (Vieillot, 1818) & * & & ExM, Rv/sP, Rv/sNP, Fol \\
\hline Myiopagis gaimardii (d'Orbigny, 1839) & & & ExM, Rv/sP, Rv/sNP, Fol, ASI \\
\hline Myiopagis caniceps (Swainson, 1835) & * & & $\mathrm{Rv} / \mathrm{sP}, \mathrm{Rv} / \mathrm{sNP}, \mathrm{Fol}$ \\
\hline Myiopagis viridicata (Vieillot, 1817) & & INTRA & ExM, Rv/sP, Rv/sNP, Fol, ASI \\
\hline Capsiempis flaveola (Lichtenstein, 1823) & & INTRA & ExM, Rv/sP, Rv/sNP, Fol \\
\hline Phaeomyias murina (Spix, 1825) & & & ExM, Rv/sP, Rv/sNP, Fol, ASI \\
\hline [Phyllomyias reiseri Hellmayr, 1905] & ${ }^{*} \mathrm{DD}(\mathrm{BL})$ & & $\mathrm{Rv} / \mathrm{sP}$ \\
\hline [Phyllomyias fascitaus (Thunberg, 1822)] & * & & $\mathrm{Rv} / \mathrm{sP}$ \\
\hline Polystictus pectoralis (Vieillot, 1817) & ${ }^{*} \mathrm{NT}(\mathrm{GL}), \mathrm{NT}(\mathrm{BL})$ & & $\mathrm{Rv} / \mathrm{sP}, \mathrm{Rv} / \mathrm{sNP}, \mathrm{Fol}$ \\
\hline Pseudocolopteryx sclateri (Oustalet, 1892) & * & & ExM, Rv/sP, Fol \\
\hline Pseudocolopteryx acutipennis (Sclater \& Salvin, 1873) & * & INTRA & ExM, Rv/sP, Fol \\
\hline Pseudocolopteryx flaviventris (d'Orbigny \& Lafresnaye, 1837) & & INTRA & Fol \\
\hline Serpophaga nigricans (Vieillot, 1817) & & & Fol \\
\hline Serpophaga subcristata (Vieillot, 1817) & & & ExM, Rv/sP, Rv/sNP, Fol \\
\hline Serpophaga griseicapilla Straneck, 2008 & & INTRA & Fol, ASI \\
\hline [Attila phoenicurus Pelzeln, 1868] & $*$ & INTRA & $\mathrm{Rv} / \mathrm{sP}$ \\
\hline [Attila cinnamomeus (Gmelin, 1789)] & * & & $\mathrm{Rv} / \mathrm{sP}, \mathrm{Rv} / \mathrm{sNP}$ \\
\hline Attila bolivianus Lafresnaye, 1848 & * & & ExM, Rv/sP, Rv/sNP, Fol, ASI \\
\hline Attila spadiceus (Gmelin, 1789) & & & $\mathrm{Rv} / \mathrm{sP}, \mathrm{ASI}$ \\
\hline Legatus leucophaius (Vieillot, 1818) & & & ExM, Rv/sP, Rv/sNP, Fol, ASI \\
\hline Myiarchus tuberculifer (d'Orbigny \& Lafresnaye, 1837) & * & & ExM, Rv/sP, Fol, ASI \\
\hline Myiarchus swainsoni Cabanis \& Heine, 1859 & & INTRA & ExM, Rv/sP, Rv/sNP, Fol \\
\hline Myiarchus ferox (Gmelin, 1789) & & & ExM, Rv/sP, Rv/sNP, Fol, ASI \\
\hline Myiarchus tyrannulus (Statius Muller, 1776) & & & ExM, Rv/sP, Rv/sNP, Fol, ASI \\
\hline Sirystes sibilator (Vieillot, 1818) & * & & ExM, Rv/sP, Rv/sNP, Fol, ASI \\
\hline Rhytipterna simplex (Lichtenstein, 1823) & * & & Fol, ASI \\
\hline Casiornis rufus (Vieillot, 1816) & * & & ExM, Rv/sP, Rv/sNP, Fol, ASI \\
\hline Pitangus sulphuratus (Linnaeus, 1766) & & INTRA & ExM, Rv/sP, Rv/sNP, Fol, ASI \\
\hline Philohydor lictor (Lichtenstein, 1823) & & & ExM, Rv/sP, Rv/sNP, Fol, ASI \\
\hline Machetornis rixosa (Vieillot, 1819) & & & ExM, Rv/sP, Rv/sNP, Fol, ASI \\
\hline Myiodynastes maculatus (Statius Muller, 1776) & & INTRA & ExM, Rv/sP, Rv/sNP, Fol, ASI \\
\hline Tyrannopsis sulphurea (Spix, 1825) & & & Fol \\
\hline Megarynchus pitangua (Linnaeus, 1766) & & & ExM, Rv/sP, Rv/sNP, Fol, ASI \\
\hline Myiozetetes cayanensis (Linnaeus, 1766) & & INTRA & ExM, Rv/sP, Rv/sNP, Fol, ASI \\
\hline Myiozetetes similis (Spix, 1825) & & INTRA & ExM, Rv/sP, Rv/sNP, \\
\hline Tyrannus albogularis Burmeister, 1856 & & INTRA & $\mathrm{Rv} / \mathrm{sP}, \mathrm{Rv} / \mathrm{sNP}$, Fol. ASI \\
\hline Tyrannus melancholicus Vieillot, 1819 & & INTRA & ExM, Rv/sP, Rv/sNP, Fol, ASI \\
\hline Tyrannus savana Daudin, 1802 & & INTRA & ExM, Rv/sP, Rv/sNP, Fol, ASI \\
\hline Tyrannus tyrannus (Linnaeus, 1758) & * & INTER & $\mathrm{Rv} / \mathrm{sP}, \mathrm{Rv} / \mathrm{sNP}, \mathrm{Fol}$ \\
\hline Griseotyrannus aurantioatrocristatus (d'Orbigny \& Lafresnaye, 1837) & & INTRA & ExM, Rv/sP, Rv/sNP, Fol \\
\hline Empidonomus varius (Vieillot, 1818) & & INTRA & ExM, Rv/sP, Rv/sNP, Fol, ASI \\
\hline
\end{tabular}




\begin{tabular}{|c|c|c|c|}
\hline \multirow{2}{*}{ Taxon } & \multicolumn{2}{|c|}{ Status } & \multirow{2}{*}{ Evidence } \\
\hline & Conservation & Migration & \\
\hline [Conopias trivirgatus (Wied, 1831)] & & & $\mathrm{Rv} / \mathrm{sP}$ \\
\hline Guyramemua affine (Burmeister, 1856) & $\mathrm{DD}(\mathrm{BL})$ & & ExM, Rv/sP, FoP \\
\hline Sublegatus modestus (Wied, 1831) & & INTRA & ExM, Rv/sP, Rv/sNP, Fol, ASI \\
\hline Colonia colonus (Vieillot, 1818) & & & ExM, Rv/sP, Rv/sNP, Fol, ASI \\
\hline Arundinicola leucocephala (Linnaeus, 1764) & & & ExM, Rv/sP, Rv/sNP, Fol, ASI \\
\hline Fluvicola albiventer (Spix, 1825) & & INTRA & ExM, Rv/sP, Rv/sNP, Fol, ASI \\
\hline Fluvicola nengeta (Linnaeus, 1766) & & & Rv/sP, Fol, ASI \\
\hline Pyrocephalus rubinus (Boddaert, 1783) & & INTRA & ExM, Rv/sP, Rv/sNP, Fol, ASI \\
\hline Gubernetes yetapa (Vieillot, 1818) & & INTRA & ExM, Rv/sP, Rv/sNP, Fol, ASI \\
\hline Alectrurus tricolor (Vieillot, 1816) & VU (GL), VU (BL) & INTRA & $\mathrm{Rv} / \mathrm{sP}, \mathrm{FoP}, \mathrm{Fol}$ \\
\hline Alectrurus risora (Vieillot, 1824) & VU (GL) & INTRA & ExM, Rv/sP \\
\hline Myiophobus fasciatus (Statius Muller, 1776) & & INTRA & ExM, Rv/sP, Rv/sNP, Fol \\
\hline Cnemotriccus fuscatus (Wied, 1831) & * & & ExM, Rv/sP, Rv/sNP, Fol, ASI \\
\hline Lathrotriccus euleri (Cabanis, 1868) & & INTRA & ExM, Rv/sP, Rv/sNP, Fol \\
\hline Empidonax alnorum Brewster, 1895 & & INTER & ExM, Rv/sP, FoP, Fol \\
\hline Contopus cinereus (Spix, 1825) & & INTRA & $\mathrm{Rv} / \mathrm{sP}, \mathrm{Rv} / \mathrm{sNP}, \mathrm{Fol}$ \\
\hline Satrapa icterophrys (Vieillot, 1818) & & & ExM, Rv/sP, Rv/sNP, Fol \\
\hline Hymenops perspicillatus (Gmelin, 1789) & & INTRA & $\mathrm{Rv} / \mathrm{sP}, \mathrm{Rv} / \mathrm{sNP}, \mathrm{Fol}$ \\
\hline Knipolegus lophotes Boie, 1828 & & & Fol \\
\hline Knipolegus striaticeps (d'Orbigny \& Lafresnaye, 1837) & * & INTRA & ExM, Rv/sP, Fol \\
\hline Knipolegus hudsoni Sclater, 1872 & & INTRA & ExM, Rv/sP, Fol \\
\hline Xolmis irupero (Vieillot, 1823) & & & ExM, Rv/sP, Rv/sNP, Fol, ASI \\
\hline Xolmis velatus (Lichtenstein, 1823) & & & ExM, Rv/sP, Rv/sNP, Fol, ASI \\
\hline Nengetus cinereus (Vieillot, 1816) & * & INTRA & ExM, Rv/sP, Rv/sNP, Fol \\
\hline \multicolumn{4}{|l|}{ Vireonidae } \\
\hline Cyclarhis gujanensis (Gmelin, 1789) & & & ExM, Rv/sP, Rv/sNP, Fol, ASI \\
\hline Hylophilus amaurocephalus (Nordmann, 1835) & & & ExM, Rv/sP \\
\hline Hylophilus pectoralis Sclater, 1866 & & & ExM, Rv/sP, Rv/sNP, Fol, ASI \\
\hline Vireo olivaceus (Linnaeus, 1766) & & INTER & ExM, Rv/sP \\
\hline Vireo chivi (Vieillot, 1817) & & INTRA & ExM, Rv/sP, Rv/sNP, Fol \\
\hline \multicolumn{4}{|l|}{ Corvidae } \\
\hline Cyanocorax cyanomelas (Vieillot, 1818) & & & ExM, Rv/sP, Rv/sNP, Fol, ASI \\
\hline Cyanocorax cristatellus (Temminck, 1823) & & & $\mathrm{Rv} / \mathrm{sP}$, Fol \\
\hline Cyanocorax chrysops (Vieillot, 1818) & * & & ExM, Rv/sP, Rv/sNP, Fol, ASI \\
\hline \multicolumn{4}{|l|}{ Hirundinidae } \\
\hline Pygochelidon cyanoleuca (Vieillot, 1817) & & INTRA & ExM, Rv/sP, Rv/sNP, Fol \\
\hline Alopochelidon fucata (Temminck, 1822) & & INTRA & $\mathrm{Rv} / \mathrm{sP}, \mathrm{Fol}$ \\
\hline Stelgidopteryx ruficollis (Vieillot, 1817) & & INTRA & ExM, Rv/sP, Rv/sNP, Fol, ASI \\
\hline Progne tapera (Vieillot, 1766) & & INTRA & ExM, Rv/sP, Rv/sNP, Fol, ASI \\
\hline Progne subis (Linnaeus, 1758) & & INTER & ExM, Rv/sP, Rv/sNP, Fol \\
\hline Progne chalybea (Gmelin, 1789) & * & INTRA & ExM, Rv/sP, Rv/sNP, Fol, ASI \\
\hline Progne elegans Baird, 1865 & & INTRA & $\mathrm{Rv} / \mathrm{sP}, \mathrm{Fol}, \mathrm{ASI}$ \\
\hline Tachycineta albiventer (Boddaert, 1783) & & INTRA & ExM, Rv/sP, Rv/sNP, Fol, ASI \\
\hline Tachycineta leucorrhoa (Vieillot, 1817) & & INTRA & ExM, Rv/sP, Rv/sNP, Fol \\
\hline Riparia riparia (Linnaeus, 1758) & * & INTER & ExM, Rv/sP, Rv/sNP, Fol \\
\hline Hirundo rustica Linnaeus, 1758 & * & INTER & ExM, Rv/sP, Rv/sNP, Fol \\
\hline Petrochelidon pyrrhonota (Vieillot, 1817) & & INTER & ExM, Rv/sP, Rv/sNP, Fol \\
\hline \multicolumn{4}{|l|}{ Troglodytidae } \\
\hline Troglodytes musculus Naumann, 1823 & & & ExM, Rv/sP, Rv/sNP, Fol, ASI \\
\hline Campylorhynchus turdinus (Wied, 1831) & * & & ExM, Rv/sP, Rv/sNP, Fol, ASI \\
\hline Pheugopedius genibarbis (Swainson, 1838) & & & ExM, Rv/sP, Rv/sNP, Fol, ASI \\
\hline Cantorchilus leucotis (Lafresnaye, 1845) & * & & ExM, Rv/sP, Rv/sNP, Fol, ASI \\
\hline Cantorchilus guarayanus (d'Orbigny \& Lafresnaye, 1837) & * & & ExM, Rv/sP, Rv/sNP, Fol, ASI \\
\hline \multicolumn{4}{|l|}{ Polioptilidae } \\
\hline Polioptila dumicola (Vieillot, 1817) & & & ExM, Rv/sP, Rv/sNP, Fol, ASI \\
\hline \multicolumn{4}{|l|}{ Donacobiidae } \\
\hline Donacobius atricapilla (Linnaeus, 1766) & & & ExM, Rv/sP, Rv/sNP, Fol, ASI \\
\hline \multicolumn{4}{|l|}{ Turdidae } \\
\hline Catharus fuscescens (Stephens, 1817) & * & INTER & ExM, Rv/sP, FoP, Fol \\
\hline Turdus leucomelas Vieillot, 1818 & & & ExM, Rv/sP, Rv/sNP, Fol, ASI \\
\hline
\end{tabular}




\begin{tabular}{|c|c|c|c|}
\hline \multirow{2}{*}{ Taxon } & \multicolumn{2}{|c|}{ Status } & \multirow{2}{*}{ Evidence } \\
\hline & Conservation & Migration & \\
\hline Turdus fumigatus Lichtenstein, 1823 & * & & ExM, Rv/sP \\
\hline Turdus hauxwelli Lawrecen, 1869 & * & & ExM, Rv/sP, Fol, ASI \\
\hline Turdus rufiventris Vieillot, 1818 & & & ExM, Rv/sP, Rv/sNP, Fol, ASI \\
\hline Turdus amaurochalinus Cabanis, 1850 & & INTRA & ExM, Rv/sP, Rv/sNP, Fol, ASI \\
\hline Turdus subalaris (Seebohm, 1887) & & INTRA & $\mathrm{Rv} / \mathrm{sP}, \mathrm{FoP}, \mathrm{Fol}$ \\
\hline Turdus albicollis Vieillot, 1818 & & & $\mathrm{ExM}, \mathrm{Rv} / \mathrm{sP}$ \\
\hline \multicolumn{4}{|l|}{ Mimidae } \\
\hline Mimus saturninus (Lichtenstein, 1823) & & & $\mathrm{ExM}, \mathrm{Rv} / \mathrm{sP}, \mathrm{Rv} / \mathrm{sNP}$, Fol \\
\hline Mimus triurus (Vieillot, 1818) & & INTRA & $\mathrm{ExM}, \mathrm{Rv} / \mathrm{sP}, \mathrm{Rv} / \mathrm{sN}$, Fol \\
\hline \multicolumn{4}{|l|}{ Estrildidae } \\
\hline Estrilda astrild (Linnaeus, 1758) & & & $\mathrm{Rv} / \mathrm{sP}, \mathrm{Rv} / \mathrm{sNP}$, Fol \\
\hline \multicolumn{4}{|l|}{ Passeridae } \\
\hline Passer domesticus (Linnaeus, 1758) & & & $\mathrm{Rv} / \mathrm{sP}, \mathrm{Rv} / \mathrm{sNP}, \mathrm{Fol}, \mathrm{ASI}$ \\
\hline \multicolumn{4}{|l|}{ Motacillidae } \\
\hline Anthus chii Vieillot, 1818 & & & ExM, Rv/sP, Rv/sNP, Fol, ASI \\
\hline \multicolumn{4}{|l|}{ Fringillidae } \\
\hline Spinus magellanicus (Vieillot, 1805) & & & $\mathrm{ExM}, \mathrm{Rv} / \mathrm{sP}, \mathrm{Rv} / \mathrm{sNP}$, Fol \\
\hline Euphonia chlorotica (Linnaeus, 1766) & & & ExM, Rv/sP, Rv/sNP, Fol, ASI \\
\hline Euphonia violacea (Linnaeus, 1758) & * & & ExM, Rv/sP, Fol \\
\hline Euphonia laniirostris d'Orbigny \& Lafresnaye, 1837 & * & & ExM, Rv/sP, Fol, ASI \\
\hline \multicolumn{4}{|l|}{ Passerellidae } \\
\hline Ammodramus humeralis (Bosc, 1792) & & & ExM, Rv/sP, Rv/sNP, Fol, ASI \\
\hline Arremon taciturnus (Hermann, 1783) & & & $\mathrm{Rv} / \mathrm{sP}, \mathrm{Fol}, \mathrm{ASI}$ \\
\hline Arremon flavirostris Swainson, 1838 & & & ExM, Rv/sP, Rv/sNP, Fol, ASI \\
\hline Zonotrichia capensis (Statius Muller, 1776) & & & ExM, Rv/sP, Rv/sNP, Fol, ASI \\
\hline \multicolumn{4}{|l|}{ Icteridae } \\
\hline Dolichonyx oryzivorus (Linnaeus, 1758) & * & INTER & $\mathrm{ExM}, \mathrm{Rv} / \mathrm{sP}, \mathrm{Rv} / \mathrm{sN}$, Fol \\
\hline Leistes superciliaris (Bonaparte, 1850) & * & INTRA & $\mathrm{ExM}, \mathrm{Rv} / \mathrm{sP}, \mathrm{Rv} / \mathrm{sNP}$, Fol \\
\hline Psarocolius decumanus (Pallas, 1769) & & & ExM, Rv/sP, Rv/sNP, Fol, ASI \\
\hline Cacicus solitarius (Vieillot, 1816) & & & ExM, Rv/sP, Rv/sNP, Fol, ASI \\
\hline Cacicus chrysopterus (Vigors, 1825) & & & ExM, Rv/sP, Rv/sNP, Fol, ASI \\
\hline Cacicus cela (Linnaeus, 1758) & * & & ExM, Rv/sP, Rv/sNP, Fol, ASI \\
\hline Cacicus haemorrhous (Linnaeus, 1766) & * & & $\mathrm{Rv} / \mathrm{sP}, \mathrm{Rv} / \mathrm{sNP}, \mathrm{Fol}, \mathrm{ASI}$ \\
\hline Icterus croconotus (Wagler, 1829) & * & & ExM, Rv/sP, Rv/sNP, Fol, ASI \\
\hline Icterus pyrrhopterus (Vieillot, 1819) & & & ExM, Rv/sP, Rv/sNP, Fol, ASI \\
\hline Molothrus rufoaxillaris Cassin, 1866 & & & ExM, Rv/sP, Rv/sNP, Fol, ASI \\
\hline Molothrus oryzivorus (Gmelin, 1788) & * & & ExM, Rv/sP, Rv/sNP, Fol, ASI \\
\hline Molothrus bonariensis (Gmelin, 1789) & & & ExM, Rv/sP, Rv/sNP, Fol, ASI \\
\hline Amblyramphus holosericeus (Scopoli, 1786) & & & ExM, Rv/sP, Rv/sNP, Fol, ASI \\
\hline Gnorimopsar chopi (Vieillot, 1819) & & & ExM, Rv/sP, Rv/sNP, Fol, ASI \\
\hline Agelaioides badius (Vieillot, 1819) & & & ExM, Rv/sP, Rv/sNP, Fol, ASI \\
\hline Agelasticus cyanopus (Vieillot, 1819) & & & ExM, Rv/sP, Rv/sNP, Fol, ASI \\
\hline Chrysomus ruficapillus (Vieillot, 1819) & & INTRA & $\mathrm{ExM}, \mathrm{Rv} / \mathrm{sP}, \mathrm{Rv} / \mathrm{sN}$, Fol \\
\hline Pseudoleistes guirahuro (Vieillot, 1819) & & & $\mathrm{Rv} / \mathrm{sP}, \mathrm{Fol}$ \\
\hline \multicolumn{4}{|l|}{ Parulidae } \\
\hline Geothlypis agilis (Wilson, 1812) & & INTER & ExM, Rv/sP \\
\hline Geothlypis aequinoctialis (Gmelin, 1789) & * & & $\mathrm{ExM}, \mathrm{Rv} / \mathrm{sP}, \mathrm{Rv} / \mathrm{sNP}$, Fol \\
\hline Setophaga pitiayumi (Vieillot, 1817) & & & $\mathrm{ExM}, \mathrm{Rv} / \mathrm{sP}, \mathrm{Rv} / \mathrm{sN}$, Fol \\
\hline Myiothlypis leucophrys Pelzeln, 1868 & & & ExM, Rv/sP \\
\hline Myiothlypis flaveola (Baird, 1865) & * & & ExM, Rv/sP, Rv/sNP, Fol, ASI \\
\hline Basileuterus culicivorus (Deppe, 1830) & & & ExM, Rv/sP, Rv/sNP, Fol, ASI \\
\hline \multicolumn{4}{|l|}{ Cardinalidae } \\
\hline Piranga flava (Vieillot, 1822) & & & ExM, Rv/sP, Fol \\
\hline Pheucticus aureoventris (d'Orbigny \& Lafresnaye, 1837) & & INTRA & ExM, Rv/sP, FoP, Fol \\
\hline Amaurospiza moesta (Hartlaub, 1853) & * & & ExM, Rv/sP \\
\hline Cyanoloxia brissonii (Lichtenstein, 1823) & & & ExM, Rv/sP, Fol \\
\hline \multicolumn{4}{|l|}{ Thraupidae } \\
\hline Nemosia pileata (Boddaert, 1783) & & & ExM, Rv/sP, Rv/sNP, Fol, ASI \\
\hline [Coryphaspiza melanotis (Temminck, 1822)] & ${ }^{*} \mathrm{VU}(\mathrm{GL}), \mathrm{EN}(\mathrm{BL})$ & & $\mathrm{Rv} / \mathrm{sP}$ \\
\hline [Embernagra platensis (Gmelin, 1789)] & & & $\mathrm{Rv} / \mathrm{sP}$ \\
\hline
\end{tabular}




\begin{tabular}{|c|c|c|c|}
\hline \multirow{2}{*}{ Taxon } & \multicolumn{2}{|c|}{ Status } & \multirow{2}{*}{ Evidence } \\
\hline & Conservation & Migration & \\
\hline Emberizoides herbicola (Vieillot, 1817) & * & & ExM, Rv/sP, Rv/sNP, Fol \\
\hline Emberizoides ypiranganus Ihering \& Ihering, 1907 & & & Fol \\
\hline Porphyrospiza caerulescens (Wied, 1830) & ${ }^{*} \mathrm{NT}(\mathrm{GL})$ & & $\mathrm{ExM}, \mathrm{Rv} / \mathrm{sP}$ \\
\hline Hemithraupis guira (Linnaeus, 1766) & * & & ExM, Rv/sP, Rv/sNP, Fol \\
\hline Tersina viridis (Illiger, 1811) & & INTRA & ExM, Rv/sP, Rv/sNP, Fol \\
\hline [Cyanerpes caeruleus (Linnaeus, 1758)] & & & $\mathrm{Rv} / \mathrm{sP}$ \\
\hline Cyanerpes cyaneus (Linnaeus, 1766) & & & $\mathrm{Rv} / \mathrm{sP}, \mathrm{Fol}$ \\
\hline Dacnis cayana (Linnaeus, 1766) & & & $\mathrm{Rv} / \mathrm{sP}, \mathrm{ASI}$ \\
\hline Saltatricula atricollis Vieillot, 1817 & & & ExM, Rv/sP, Rv/sNP, Fol, ASI \\
\hline Saltatricula multicolor (Burmeister, 1860) & & INTRA & $\mathrm{Rv} / \mathrm{sP}, \mathrm{Fol}$ \\
\hline Saltator maximus (Statius Muller, 1776) & * & & $\mathrm{Rv} / \mathrm{sP}$, Fol, ASI \\
\hline Saltator coerulescens Vieillot, 1817 & * & & ExM, Rv/sP, Rv/sNP, Fol, ASI \\
\hline Saltator similis d'Orbigny \& Lafresnaye, 1837 & * & & $\mathrm{ExM}, \mathrm{Rv} / \mathrm{sP}, \mathrm{Rv} / \mathrm{sNP}$ \\
\hline Saltator aurantiirostris Vieillot, 1817 & & & ExM, Rv/sP, Rv/sNP, Fol, ASI \\
\hline Coereba flaveola (Linnaeus, 1758) & & & ExM, Rv/sP, Rv/sNP, Fol, ASI \\
\hline Asemospiza obscura (d'Orbigny \& Lafresnaye, 1837) & & INTRA & ExM, Rv/sP \\
\hline Asemospiza fuliginosa (Wied, 1830) & & & $\mathrm{Rv} / \mathrm{sP}, \mathrm{Fol}$ \\
\hline Volatinia jacarina (Linnaeus, 1766) & & INTRA & ExM, Rv/sP, Rv/sNP, Fol. ASI \\
\hline Eucometis penicillata (Spix, 1825) & & & ExM, Rv/sP, Rv/sNP, Fol, ASI \\
\hline [Trichothraupis melanops (Vieillot, 1818)] & & & $\mathrm{Rv} / \mathrm{sP}$ \\
\hline Loriotus luctuosus (d’0rbigny \& Lafresnaye, 1837) & * & & Fol \\
\hline Coryphospingus cucullatus (Statius Muller, 1776) & & & ExM, Rv/sP, Rv/sNP, Fol, ASI \\
\hline Tachyphonus rufus (Boddaert, 1783) & & & ExM, Rv/sP, Rv/sNP, Fol, ASI \\
\hline Tachyphonus coronatus (Vieillot, 1822) & & & ExM, Rv/sP \\
\hline Ramphocelus carbo (Pallas, 1764) & & & ExM, Rv/sP, Rv/sNP, Fol, ASI \\
\hline Sporophila lineola (Linnaeus, 1758) & & INTRA & ExM, Rv/sP, Rv/sNP, Fol, ASI \\
\hline Sporophila plumbea (Wied, 1830) & & INTRA & $\mathrm{Rv} / \mathrm{sP}, \mathrm{Rv} / \mathrm{sNP}, \mathrm{Fol}$ \\
\hline Sporophila collaris (Boddaert, 1783) & & & ExM, Rv/sP, Rv/sNP, Fol, ASI \\
\hline Sporophila nigricollis (Vieillot, 1823) & & INTRA & $\mathrm{Rv} / \mathrm{sP}, \mathrm{Rv} / \mathrm{sNP}, \mathrm{Fol}$ \\
\hline Sporophila caerulescens (Vieillot, 1823) & & INTRA & ExM, Rv/sP, Rv/sNP, Fol \\
\hline Sporophila leucoptera (Vieillot, 1817) & & INTRA & ExM, Rv/sP, Rv/sNP, Fol, ASI \\
\hline Sporophila nigrorufa (d'Orbigny \& Lafresnaye, 1837) & ${ }^{*} \mathrm{VU}(\mathrm{GL}), \mathrm{VU}(\mathrm{BL})$ & INTRA & ExM, Rv/sP, FoP \\
\hline Sporophila bouvreuil (Statius Muller, 1776) & * & INTRA & $\mathrm{Rv} / \mathrm{sP}, \mathrm{Fol}$ \\
\hline Sporophila pileata (Sclater, 1865) & *NT (BL) & INTRA & ExM, Rv/sP, Fol \\
\hline Sporophila hypoxantha Cabanis, 1851 & $\mathrm{VU}(\mathrm{BL})$ & INTRA & ExM, Rv/sP, Rv/sNP, Fol, ASI \\
\hline Sporophila ruficollis Cabanis, 1851 & ${ }^{*} \mathrm{NT}(\mathrm{GL}), \mathrm{VU}(\mathrm{BL})$ & INTRA & $\mathrm{Rv} / \mathrm{sP}, \mathrm{Rv} / \mathrm{sNP}, \mathrm{FoP}$, Fol \\
\hline Sporophila iberaensis Di Giacomo \& Kopuchian, 2016 & * EN (GL) & INTRA & Fol \\
\hline Sporophila palustris (Barrows, 1883) & ${ }^{*} \mathrm{EN}(\mathrm{GL}), \mathrm{VU}(\mathrm{BL})$ & INTRA & $\mathrm{Rv} / \mathrm{sP}, \mathrm{Fol}$ \\
\hline Sporophila hypochroma Todd, 1915 & *NT (GL) & INTRA & $\mathrm{Rv} / \mathrm{sP}, \mathrm{Fol}$ \\
\hline Sporophila cinnamomea (Lafresnaye, 1839) & *VU (GL), NR (BL) & INTRA & $\mathrm{Rv} / \mathrm{sP}, \mathrm{Rv} / \mathrm{sNP}, \mathrm{Fol}$ \\
\hline Sporophila angolensis (Linnaeus, 1766) & & & ExM, Rv/sP, Rv/sNP, Fol, ASI \\
\hline Sporophila maximiliani (Cabanis, 1851) & ${ }^{*} \mathrm{EN}(\mathrm{GL}), \mathrm{CR}(\mathrm{BL})$ & INTRA & $\mathrm{Rv} / \mathrm{sP}$ \\
\hline Thlypopsis sordida (d'Orbigny \& Lafresnaye, 1837) & & INTRA & ExM, Rv/sP, Rv/sNP, Fol \\
\hline Cypsnagra hirundinacea (Lesson, 1831) & & & ExM, Rv/sP, FoP, Fol, ASI \\
\hline Microspingus melanoleucus (d'Orbigny \& Lafresnaye, 1837) & & & ExM, Rv/sP, Rv/sNP, Fol, ASI \\
\hline Microspingus cinereus Bonaparte, 1850 & & & ExM \\
\hline Conirostrum speciosum (Temminck, 1824) & * & & ExM, Rv/sP, Rv/sNP, Fol, ASI \\
\hline Sicalis citrina Pelzeln, 1870 & & INTRA & $\mathrm{Rv} / \mathrm{sP}, \mathrm{Fol}$ \\
\hline Sicalis flaveola (Linnaeus, 1766) & & & ExM, Rv/sP, Rv/sNP, Fol, ASI \\
\hline Sicalis luteola (Sparrman, 1789) & & INTRA & ExM, Rv/sP, Rv/sNP, Fol \\
\hline Pipraeidea melanonota (Vieillot, 1819) & & & $\mathrm{Rv} / \mathrm{sP}, \mathrm{Fol}$ \\
\hline Neothraupis fasciata (Lichtenstein, 1823) & *NT (GL) & & ExM, Rv/sP \\
\hline Cissopis leverianus (Gmelin, 1788) & * NT (GL) & & $\mathrm{Rv} / \mathrm{sP}, \mathrm{Fol}$ \\
\hline Schistochlamys melanopis (Latham, 1790) & & & ExM, Rv/sP, FoP, Fol \\
\hline [Schistochlamys ruficapillus (Vieillot, 1817)] & & & $\mathrm{Rv} / \mathrm{sP}$ \\
\hline Paroaria coronata (Miller, 1776) & & & ExM, Rv/sP, Rv/sNP, Fol, ASI \\
\hline Paroaria capitata (d'0rbigny \& Lafresnaye, 1837) & & & ExM, Rv/sP, Rv/sNP, Fol, ASI \\
\hline Thraupis sayaca (Linnaeus, 1766) & & & ExM, Rv/sP, Rv/sNP, Fol, ASI \\
\hline Thraupis palmarum (Wied, 1821) & & & ExM, Rv/sP, Rv/sNP, Fol \\
\hline Stilpnia cayana (Linnaeus, 1766) & & & ExM, Rv/sP, Rv/sNP, \\
\hline Tangara mexicana (Linnaeus, 1766) & * & & Fol \\
\hline
\end{tabular}


Table 2. Tertiary list of birds related to the Pantanal, Brazil.

\begin{tabular}{|c|c|}
\hline Taxon & Citation source / Reason for exclusion \\
\hline Crypturellus soui (Hermann, 1783) & Donatelli (2005) / A \\
\hline Nothura boraquira (Spix, 1825) & Straube et al. (2006a), Benites et al. (2017) / A \\
\hline Spatula cyanoptera (Vieillot, 1816) & Nunes et al. (2008) / C \\
\hline Mareca sibilatrix (Poeppig, 1829) & Nunes (2011a) / A \\
\hline Penelope obscura Temminck, 1815 & Aguirre \& Aldrighi (1983), Souza (2005), Cintra (2014) / C \\
\hline Odontophorus capueira (Spix, 1825) & Cintra (2014) / D \\
\hline Patagioenas plumbea (Vieillot, 1818) & Leuzinger (2011) / B \\
\hline Piaya melanogaster (Vieillot, 1817) & Nunes (2010) / A \\
\hline Hydropsalis climacocerca (Tschudi, 1844) & Aguirre \& Aldrighi (1983), Cintra (2014) / A \\
\hline Hydropsalis forcipata (Nitzsch, 1840) & Nunes (2011a) / C \\
\hline Florisuga fusca (Vieillot, 1817) & Nunes \& Tomas (2004b) / C \\
\hline Anopetia gounellei (Boucard, 1891) & Naumburg et al. (1930) / C \\
\hline Phaethornis eurynome (Lesson, 1832) & Araújo (2001), Tubelis \& Tomas (2003a), Nunes et al. (2009), Cintra (2014) / C \\
\hline Thalurania glaucopis (Gmelin, 1788) & Tubelis \& Tomas (2003a) / A \\
\hline Taphrospilus hypostictus (Gould, 1862) & Sick (1997) / C \\
\hline Elliotomyia chionogaster (Tschudi, 1845) & Lopes et al. (2016) / A \\
\hline Opisthocomus hoazin (Statius Muller, 1776) & Brown-Jr. (1986) / A \\
\hline Tigrisoma fasciatum (Such, 1825) & Nunes (2010) / B \\
\hline Geranoaetus polyosoma (Quoy \& Gaimard, 1824) & Reichholf (1976) / A \\
\hline Glaucidium minutissimum (Wied, 1830): & Pinho (2005) / A \\
\hline Trogon viridis Linnaeus, 1766 & Cintra (2014) / D \\
\hline Trogon surrucura Vieillot, 1817 & Tubelis \& Tomas (2003a), Pinho (2005), Nunes et al. (2009), Nunes (2010) / A \\
\hline Trogon collaris Vieillot, 1817 & Cintra (2014) / D \\
\hline Ramphastos tucanus Linnaeus 1758 & Pivatto et al. (2008) / C \\
\hline Selenidera maculirostris (Lichtenstein, 1823) & Tubelis \& Tomas (2003a), Cintra (2014) / C \\
\hline Veniliornis spilogaster (Wagler, 1827) & Leuzinger (2011) / A \\
\hline Campephilus robustus (Lichtenstein, 1818) & Cintra (2014) / D \\
\hline Piculus leucolaemus (Natterer \& Malherbe, 1845) & Lopes et al. (2016) / A \\
\hline Piculus aurulentus (Temminck, 1821) & Schubart et al. (1965), Cintra (2014) / A \\
\hline Anodorhynchus glaucus (Vieillot, 1816) & Collar et al. (1992) / A \\
\hline Myrmophylax atrothorax (Boddaert, 1783) & Pinto (1938), Cintra (2014) / D \\
\hline Myrmotherula axillaris (Vieillot, 1817) & Cintra (2014) / D \\
\hline Myrmotherula menetriesii (d'Orbigny, 1837) & Tubelis \& Tomas (2003a), Cintra (2014) / A \\
\hline Thamnophilus torquatus Swainson, 1825 & Donatelli (2005), Brandão et al. (2011) / C \\
\hline Thamnophilus punctatus (Shaw, 1809) & Nunes (2010), Brandão et al. (2011), Leuzinger (2011) / B \\
\hline Pyriglena leucoptera (Vieillot, 1818) & Travassos \& Freitas (1940), Leuzinger (2011) / C \\
\hline Hypocnemis ochrogyna Zimmer, 1932 & Cintra (2014) / D \\
\hline Willisornis poecilinotus (Cabanis, 1847) & Cintra (2014) / D \\
\hline Xiphocolaptes albicollis (Vieillot, 1818) & Leuzinger (2011), Cintra (2014) / A \\
\hline Xiphorhynchus fuscus (Vieillot, 1818) & Donatelli (2005) / C \\
\hline Lepidocolaptes albolineatus (Lafresnaye, 1845) & Tubelis \& Tomas (2003a) / A \\
\hline Lochmias nematura (Lichtenstein, 1823) & Leuzinger (2011) / B \\
\hline Anabacerthia lichtensteini (Cabanis \& Heine, 1859) & Leuzinger (2011) / A \\
\hline Syndactyla dimidiata (Pelzeln, 1859) & Brown-Jr. (1986), Tubelis \& Tomas (2003a), Leuzinger (2011), Cintra (2014) / A \\
\hline Automolus leucophthalmus (Wied, 1821) & Nunes et al. (2008) / C \\
\hline Synallaxis gujanensis (Gmelin, 1789) & Cintra (2014) / D \\
\hline Schiffornis virescens (Lafresnaye, 1838) & Leuzinger (2011) / A \\
\hline Schiffornis turdina (Wied, 1831) & Lopes et al. (2016) / A \\
\hline Neopipo cinnamomea (Lawrence, 1869) & Donatelli et al. (2014) / C \\
\hline Poecilotriccus plumbeiceps (Lafresnaye, 1846) & Nunes et al. (2010), Cintra (2014) / D \\
\hline Myiornis auricularis (Vieillot, 1818) & Nunes et al. (2008) / C \\
\hline Myiopagis flavivertex (Sclater, 1887) & Schubart et al. (1965), Tubelis \& Tomas (2003a) / A \\
\hline Ramphotrigon ruficauda (Spix, 1825) & Cintra (2014) / D \\
\hline Fluvicola pica (Boddaert, 1783) & Nunes (2010), Leuzinger (2011) / A \\
\hline Leistes militaris (Linnaeus, 1758) & Brandão et al. (2011) / A \\
\hline Icterus jamacaii (Gmelin, 1788) & Pinho (2005) / A \\
\hline Agelasticus thilius (Molina, 1782) & Nunes (2011a) / A \\
\hline Charitospiza eucosma Oberholser, 1905 & Leuzinger (2011), Lopes et al. (2016) / B \\
\hline
\end{tabular}




\begin{tabular}{|c|c|}
\hline Taxon & Citation source / Reason for exclusion \\
\hline Lanio nattereri (Pelzeln, 1870) & Tubelis \& Tomas (2003a), Cintra (2014) / C \\
\hline Sporophila bouvronides (Lesson, 1831) & Pinho (2005) / A \\
\hline Sporophila minuta (Linnaeus 1758) & Nunes et al. (2009) / C \\
\hline Sporophila melanogaster (Pelzeln, 1870) & Silveira \& Straube (2008) / A \\
\hline Rauenia bonariensis (Gmelin, 1789) & Nunes et al. (2010) / A \\
\hline
\end{tabular}

Reason - Reason for exclusion (adapted from Pacheco et al., 2021): (A) The records for Pantanal with known authorship (documentation non-existent or not informed) are inconsistent with the species' distributional and dispersal patterns; (B) Occurrence in Pantanal either wrong, possibly non-existent or speculative. Species with marginal distribution in the Pantanal, but without evidence of occurrence in floodplain; (C) Occurrence in Pantanal either wrong, possibly non-existent or speculative. The species is listed to Pantanal; nonetheless, data on any particular record is unknown or invalid; (D) Occurrence in Pantanal either wrong, possibly non-existent or speculative. Published range map shows it in Pantanal; nonetheless, data on any particular record is unknown or invalid.

tory, for the maintenance of a meaningful avifaunistic richness. The avifauna of this region can be considered quite rich compared to that found in other wetlands of the world, such as the Okavango Delta in Botswana with more than 450 species (Hancock et al., 2007) and the "Esteros del Iberá" in Argentina with 344 species (Giraudo et al., 2003). In Brazil, 373 species are listed in the Upper Paraná River floodplain (Paraná and Mato Grosso do Sul) alone (Gimenes et al., 2007), with an additional, 230 species (Pereira \& Poerschke, 2010) listed in Lagoa do Peixe (Rio Grande do Sul).

The increasing advent of "Citizen Science" practices have made it possible to make an expressive contribution to everything that was previously known about the Pantanal avifauna, especially with the advent of digital platforms such as WikiAves and Xeno-Canto that have been fundamental in expanding knowledge and supporting documentation. However, it is emphasized that there are some limitations to the inadvertent use of this data, such as reliability in identification and inaccuracy of location, which need to be checked directly with the authors of the records. Despite notable advances in the knowledge of the composition of the Pantanal avifauna, there are great geographical gaps, notably in the regions of Cáceres, Paiaguás, and Chaco (Fig. 1), as evidenced in the studies by Frota et al. (2020b) and FernandezArellano et al. (2021), who identified a series of locations with poorly sampling effort for the bird inventory.

\section{Biogeographic affinities}

The avifauna occurring in the Pantanal floodplain is composed mostly of species that are widely distributed in other South American phytogeographic provinces as well, such as the Cerrado and Chaco regions and, to a lesser extent, in the Amazon, Bosques Secos Chiquitanos, and Atlantic forests (Nunes \& Tomas, 2004a). The similarities between the bird communities of the Pantanal region and the Cerrado region are to be expected as onethird of the Pantanal region is covered by the savanna phytophysiognomies (Ratter et al., 2003). Species with a wide distribution in the Cerrado region (Silva, 1995; Silva \& Bates, 2002) such as Penelope ochrogaster, Uropelia campestris, Alipiopsitta xanthops, Saltatricula atricollis, and Basileuterus culicivorus are also widespread in the floodplain. The Chaco province enters a few kilometers in to Brazil, notably around the municipality of Porto Murtinho, in the southern part of the Pantanal region (Prado 1993a, b; Prado \& Gibbs, 1993; Straube et al., 2006a), and the only species that can be considered endemic to this region is Saltatricula multicolor. However, there are some taxa, whose distribution is largely centered in the Upper Paraguay River Basin, notably in the lowland and western edge of the Pantanal, and which, in a way, can be considered typical of the Chaco region: Ortalis canicollis, Aratinga nenday, Nystalus striatipectus, Melanerpes cactorum, Celeus lugubris, Xiphocolaptes major, Paroaria coronata, and Microspingus melanoleucus.

Most of the typical species of the Amazon Basin (Silva, 1996) have the northern portion of the floodplain (Santo Antônio do Leverger and the Cáceres region), which serves as the southern most limit of its distribution. In this context, Tinamus tao, Pauxi tuberosa, Helicolestes hamatus, Megascops usta, Trogon melanurus, Pteroglossus inscriptus, Pteroglossus bitorquatus, Campephilus rubricollis, and Thamnophilus amazonicus are the outstanding species in this region. Other species, however, extend their southern limits to the western edge in the Serra do Amolar region (Nunes et al., 2018) such as Pseudastur albicollis, Coccycua minuta, Veniliornis affinis, and Dendroplex picus. The Bosques Secos Chiquitanos dry forests extend tangentially to the far west of Mato Grosso and Mato Grosso do Sul (Prado \& Gibbs, 1993; Timothy et al., 2006; Werneck et al., 2011). At least four species occurring in the Pantanal floodplain have distributions centered on these Chiquitanos dry woods (Vasconcelos \& Hoffmann, 2006, Nunes et al., 2018): Phaethornis subochraceus, Pyrrhura molinae, Thamnophilus sticturus, and Cantorchilus guarayanus.

\section{Migration movement}

Of the migratory species occurring in Brazil (Somenzari et al., 2018), at least a third use the Pantanal floodplain as a stopover or wintering site during migratory movements across the American continent to a greater or lesser extent. Waterfowl migratory displacements represent a significant portion of inter- and intracontinental migrants, which is expected for seasonally flooded environments (Oliveira, 2006; Nunes \& Tomas, 2008; Donatelli et al., 2017). In this context, some anatids (Coscoroba coscoroba, Callonetta leucophrys, Anas bahamensis, Spatula platalea, Netta peposaca, and Oxyura vittata) stand out, which reproduce mainly in Rio Grande do Sul, southern Bolivia, and northwestern Argentina regions, then move to the floodplain of the Pantanal during the winter and spend the dry periods in southern South America (Nunes \& Tomas, 2008). With regard 
to migrants dependent on aquatic habitats, sandpipers (genera Tringa and Calidris) stand out for their long-distance displacement from breeding areas in the tundra to wintering sites in Patagonia (Nunes \& Tomas, 2008), therefore being the Pantanal mudflats habitats a very important stopover for Scolopacidae family (Serrano, 2010; Frota et al., 2020c). The occurrence of Nearctic migratory species, such as Numenius hudsonicus, Arenaria interpres, Calidris alba, C. pusilla, C. minutilla, C. bairdii, Xema sabini, Leucophaeus pipixcan, and Chlidonias niger, can be considered accidental since the Pantanal floodplain not usual route for these species (Serrano, 2010; Kantek \& Onuma, 2013; Frota et al., 2020a).

Antas et al. (2016) reported that young Rynchops niger birds banded in the SESC Pantanal in Barão de Melgaço (MT) were recaptured in the Mar Chiquita lagoon near Mar del Plata in Argentina, and in Lagoa do Peixe (Rio Grande do Sul). After the reproductive period, which coincides with the end of the rainy season, the population of Pheucticus aureoventris disperses from the Andes pre-mountain range to the Pantanal floodplain and surrounding plateaus from May to August (Nunes, 2008).

There is a great flow of migratory birds coming from the southernmost regions of South America, notably the seedeaters (Sporophila), to central and northern Brazil. Some species (S. hypoxantha, S. caerulescens, S. iberaensis, S. palustris, and S. ruficollis) reproduce in the hydromorphic fields in the southern regions of South America and appear in large numbers in the native fields of the Pantanal during the winter (Nunes \& Tomas, 2008).

Migratory birds, notably northern ones, are among the main players involved in ecological processes of nutrient cycling and dispersion of important pathogens between the two continents (Kawamoto et al., 2005; Nunes \&Tomas, 2008; Araújo et al., 2014).

\section{Endangered species}

According to the Handbook of the Birds of the World \& BirdLife International (2020), 231 species occurring in the Pantanal region are experiencing population declines. However, most of them comprise common species with viable and vigorous populations in the floodplains, a fact that highlights the Pantanal region as an important biological refuge for birds in South America (Nunes, 2009). Of these, Rhea americana and Crax fasciolata are considered "Near Threatened" and "Vulnerable", respectively, at a global scale. Hasenclever et al. (2004) estimated 6,500 R. americana individuals across the Pantanal floodplain, whereas the estimated population ranged from 1 to 2 individuals per $\mathrm{km}^{2}$ in western Nhecolândia (Gräbin et al., 2012). In turn, the density estimates of $C$. fasciolata in western Nhecolândia varied according to habitat, between 3 and 5 individuals per hectare in open and forested areas, respectively (Nunes, 2015).

In addition to deforestation and replacement of natural landscapes by cultivated pastures (Tomas et al., 2009), extreme drought and fires (Marengo et al., 2021; Pivello et al., 2021) that have occurred in recent years have seriously threatened regional richness and diversity (Berlinck et al., 2021). The fires that occurred in the Pantanal region in 2020 destroyed 29\% of the native vegetation; more than $4 \%$ of the burned area corresponded to conservation sites (Libonati et al., 2020). Four million hectares of forest, cerrado, and savanna were burned, with the northern portion of the floodplain being the most affected by the fires (Libonati et al., 2020). It is noteworthy that the region affected by the fires coincides with the refuges of large populations of two of the most threatened species occurring in the Pantanal floodplain: the Chestnut-bellied Guan (Penelope ochrogaster) and the Hyacinth Macaw (Anodorhynchus hyacinthinus). In addition, many migratory species, notably those dependent on native grasslands habitats such as most seedeaters (Nunes \& Tomas, 2008), were also affected by the loss of feeding sites due to these fires. Based on the type of habitat they explore and nesting sites, it is speculated that more than half of the bird species that occur in the Pantanal may have had their populations affected to a higher or lower extent by the fires. However, the effects of these events on populations of endangered, migrant, and even common species in the Pantanal are still unknown. Considering the future scenario of climate change, the traditional and sustainable management of the Pantanal, sustainable fire management, and maintenance of the mosaic and spatial arrangement of the landscape units intact and continuous is essential to maintain the diversity in this unique and fragile ecosystem.

\section{ACKNOWLEDGMENTS}

We thank EMBRAPA Pantanal, WWF-Brazil, the Pantanal Research Center-CPP, CNPq, Conservation International of Brazil, EarthWatch Institute, the Manoel de Barros Foundation, and the Federal University of Mato Grosso do Sul Foundation for their financial and logistical support in the research projects and species inventories carried out by the authors in the Pantanal floodplain. To the Coordenação de Aperfeiçoamento de Pessoal de Nível Superior - Brasil (CAPES) - and the Fundação de Amparo à Pesquisa do Estado de Mato Grosso (FAPEMAT) for granting scholarships to AVBF and BDV. We also thank the countless Pantanal farm owners who allowed us to conduct studies on their properties. In particular, we thank the Vitor Piacentini and authors of photographic records available on the WikiAves digital platform for sharing their valuable information on species records. We also thank the anonymous reviewers who contributed greatly to the preparation of the final version of this article.

\section{AUTHORS' CONTRIBUTIONS}

APN, SRP, AVBF, BDV, RRL, RJD, FCS, MACP: Conceptualization, Methodology, Data curation, Writing - original draft, Investigation, Writing - review \& editing. DMMO, CB, AVM, WMT, GOF, RADS, MB, SM, RSM: Investigation. 


\section{CONFLICT OF INTEREST}

Authors declare there are no conflicts of interest and the authors alone are responsible for the content and writing of the paper.

\section{REFERENCES}

Aguirre, A.C. \& Aldrighi, A.D. 1983. Catálogo das aves do Museu da Fauna: primeira parte. Rio de Janeiro, IBDF. 143p.

Allen, J.A. 1891. On a collection of birds from Chapada, Mato Grosso, Brazil, made by Mr. Herbert H. Smith. Part I - Oscines. Bulletin of the American Museum of Natural History, 3:337-380.

Allen, J.A. 1892. On a collection of birds from Chapada, Mato Grosso, Brazil, made by Mr. Herbert H. Smith. Part II - Tyrannidae. Bulletin of the American Museum of Natural History, 4: 331-350.

Allen, J.A. 1893. On a collection of birds from Chapada, Mato Grosso, Brazil, made by Mr. Herbert H. Smith. Part III - Pipridae to Rheidae. Bulletin of the American Museum of Natural History, 5: 107-158.

Alvares, C.A.; Stape, J.L.; Sentelhas, P.C.; Moraes, G., Leonardo, J. \& Sparovek, G. 2014. Köppen's climate classification map for Brazil. Meteorologische Zeitschrift, 22(6): 711-728. DOI

Amaral, P.P. \& Ragusa-Netto, J. 2008. Bird mixed-flocks and nuclear species in a tecoma savanna in the Pantanal. Brazilian Journal of Biology, 68(3): 511-518.

Antas, P.T.Z. 1983. Migration of nearctic shorebirds (Charadriidae and Scolopacidae) in Brazil - flyways and their different seasonal use. Wader Study Group Bulletin, 39(1): 52-56.

Antas, P.T.Z. 1994. Migration and other movements among the lower Paraná River valley wetlands, Argentina, and the south Brazil/Pantanal wetlands. Bird Conservation International, 4(2): 181-190.

Antas, P.T.Z. \& Nascimento, I.L.S. 1996. Tuiuiu: sob os céus do Pantanal, biologia e conservação do Tuiuiú. São Paulo, Empresa das Artes. 169p.

Antas, P.T.Z. \& Palo-Jr., H. 2004. Guia de aves: espécies da reserva particular do patrimônio natural do SESC Pantanal. 1ª Edição. Rio de Janeiro, SESC Nacional. 246p.

Antas, P.T.Z. \& Palo-Jr., H. 2009. Guia de aves: espécies da reserva particular do patrimônio natural do SESC Pantanal. 2.ed. Rio de Janeiro, SESC Nacional.

Antas, P.T.Z.; Carrara, L.A.; Ubaid, F.K.; Oliveira-Júnior, S.B. \& Ferreira, L.P. 2016. Aves coloniais da Reserva Particular do SESC Pantanal. Conhecendo o Pantanal 10. Rio de Janeiro, SESC, Departamento Nacional. 236p.

Antas, P.T.Z.; Yamashita, C. \& Valle, M.P. 1986. First record of purple Martin (Progne subis) in Mato Grosso State, Brazil. Journal of Field Ornithology, 57(2): 171-172

Araújo, A.C. 2001. Flora, fenologia de floração e síndromes de polinização em capões do Pantanal sul mato grossense. Campinas, Universidade Estadual de Campinas, Programa de Pós-Graduação em Ecologia. Tese de Doutorado. 99p.

Araújo, J.; Azevedo-Júnior, S.M.; Gaidet, N.; Hurtado, R.F.; Walker, D.; Thomazelli, L.M.; Ometto, T.; Seixas, M.M.M.; Rodrigues, R.; Galindo, D.B.; Silva, A.C.S.; Rodrigues, A.M.M.; Bomfim, L.L.; Mota, M.A.; Larrazábal, M.E.; Branco, J.0.; Serafini, P.; Neto, I.S.; Franks, J.; Webby, R.J.; Webster, R.G. \& Durigon, E.L. 2014. Avian Influenza Virus (H11N9) in migratory shorebirds wintering in the Amazon Region, Brazil. Plos One, 9(10): e110141. DOI

Benites, M. \& Mamede, S. 2021. Avifauna do Chaco de Porto Murtinho, Mato Grosso do Sul, Brasil: implicações à conservação. In: Sartori, A.L.B.; Souza, P.R. \& Arruda, R.C.O. (Eds.). Chaco: caracterização, riqueza, diversidade, recursos e interações. Campo Grande, Editora da
Universidade Federal de Mato Grosso do Sul. https://repositorio.ufms.br/ handle/123456789/3432 (in press).

Benites, M.; Mamede, S.; Carvalho, G. \& Alho-Jr., C. 2017. Assessment of avian occurrence in the Brazilian chaco. International Journal of Avian \& Wildlife Biology, 2(4): 99-113.

Berlinck, C.N.; Lima, L.H.A.; Pereira, A.M.M.; Carvalho Jr., E.A.R.; Paula, R.C.; Tomas, W.M. \& Morato, R.G. 2021. The Pantanal is on fire and only a sustainable agenda can save the largest wetland in the world. Brazilian Journal of Biology, 82: 1-2. DOI

Brandão, L.C.; Antas, P.T.Z.; Oliveira, L.F.B.; Pádua, M.T.J.; Pereira, N.C. \& Valutky, W.W. 2011. Plano de Manejo da Reserva Particular de Patrimônio Natural do SESC Pantanal. Rio de Janeiro, SESC Departamento Nacional. $146 \mathrm{p}$.

Brazil. 2020. Cadastro Nacional de Unidades de Conservação (Painel Unidades de Conservação Brasileiras). Available: http://antigo.mma.gov.br/areasprotegidas/cadastro-nacional-deu-cs. Access: 22/03/2020.

Brown-Jr., K.S. 1986. Zoogeografia da região do Pantanal Mato-grossense. In: Simpósio sobre Recursos Naturais e Sócio-Econômicos do Pantanal, 10 Anais. Brasília, DF, EMBRAPA-DDT. p. 137-182.

Carlos, C.J.; Straube, F.C. \& Pacheco, J.F. 2010. Conceitos e definições sobre documentação de registros ornitológicos e critérios para a elaboração de listas de aves para os estados brasileiros. Revista Brasileira de Ornitologia, 18(4): 355-361.

Cestari, C. 2006a. Primeiro registro documentado de Alectrurus tricolor para 0 Pantanal. Revista Brasileira de Ornitologia, 14(2): 155-156.

Cestari, C. 2006b. Novos registros de aves do gênero Sporophila para 0 Pantanal. Atualidades Ornitológicas, 129: 7.

Chiaravalloti, R.M.; Tomas, W.M.; Tizianel, F.A.T. \& Camilo, A.R. 2009. Aves, Accipitridae, Harpyhaliaetus coronatus: a documented record in the Pantanal wetland. Check List, 5(1): 89-91.

Cintra, R. 2014. Aves do Pantanal: 523 espécies incluindo cerca de 350 da Amazônia e 450 do Cerrado. Manaus, Editora INPA. 376p.

Cintra, R. \& Yamashita, C. 1990. Hábitats, abundância e ocorrência das espécies de aves do Pantanal de Poconé, Mato Grosso, Brasil. Papéis Avulsos Zoologia, 37(1): 1-21.

Collar, N.J.; Gonzaga, L.P.; Krabbe, N.; Madroño-Nieto, A.; Naranjo, L.G.; Parker III, T.A. \& Wege, D. 1992. Threatened birds of Americas: the ICBP/IUCN red data book. Cambridge, International Council for Bird Preservation. 1150p.

Coutinho, M.; Campos, Z.; Mourão, G. \& Mauro, R. 1997. Aspectos ecológicos terrestres e semi-aquáticos no Pantanal. In: [p. 195-294]. Brasil. Ministério do Meio Ambiente, dos Recursos Hídricos e da Amazônia Legal. Plano de Conservação da Bacia do Alto Paraguai (Pantanal) - PCBAP. Diagnóstico dos meios físico e biótico: meio biótico. Brasília, Ministério do Meio Ambiente, dos Recursos Hídricos e da Amazônia Legal. v. 2, 400p.

Donatelli, R. 2005. Birds and dynamics habitat mosaics in the Pantanal. In: Chandler, M.; Wang, E. \& Johansson, P. (Eds.). The Pantanal conservation research initiative. Annual report. Boston, Earthwatch Institute. p. 50-69.

Donatelli, R.J. \& Ubaid, F.K. 2008. Primeiro registro documentado de Celeus flavus no Estado de Mato Grosso do Sul. Cotinga, 30: 85-86.

Donatelli, R.J.; Posso, S.R. \& Toledo, M.C.B. 2014. Distribution, composition and seasonality of aquatic birds in the Nhecolândia sub-region of South Pantanal, Brazil. Brazilian Journal Biology, 74(4): 844-853.

Donatelli, R.J.; Vianna, R.; Martins, R.M.; Whitacker, R.; Eaton, D.P.; Cardoso, G.S. \& Rodrigues, F.G. 2017. Temporal and spatial variation of richness and abundance of the community of birds in the Pantanal wetlands of Nhecolândia (Mato Grosso do Sul, Brazil). Revista de Biologia Tropical, 65(4): 1358-2017.

Emanuel, V. 2013. A bird in two hemispheres. Available: https://ventbirdblog. wordpress.com/2013/10. Access: 22/03/2021. 
Evangelista, M.M.; Pinho, J.B. \& Chupel, T.F. 2010. Descrição do ninho e dos ovos de Zebrilus undulatus (Gmelin, 1789) (Ciconiiformes: Ardeidae) na região do Pantanal de Poconé, Mato Grosso, Brasil. Revista Brasileira de Ornitologia, 18(2): 121-123.

Fernandez-Arellano, G.J.; Teixido, A.L.; Bernardon, B.; Bueno, E.R.; Ferreira, T.V.; Gonçalves, S.A.; Jesus, M.; Thomas, K.P.C.S.; Zucchetto, M.; Piacentini, V. \& Pinho, J.B. 2021. Knowledge gaps and biases in the Pantanal indicate future directions for ornithological research in large wetlands. Ibis, 163. DOI

Frota, A.V.B.; Vitorino, B.D.; da Silva, C.J.; Ikeda-Castrillon, S.K. \& Nunes, J.R.S. 2020a. Birds of the Ramsar site Estação Ecológica de Taiamã and buffer zone, Pantanal wetlands, Brazil. Check List, 16(2): 401-422. D0I

Frota, A.V.B.; Vitorino, B.D.; Nunes, J.R.S.; da Silva, C.J. 2020b. Main trends and gaps in studies for bird conservation in the Pantanal wetland. Neotropical Biology and Conservation, 15(4): 427-445. D01

Frota, A.V.B.; Vitorino, B.D.; da Silva, C.J.; Ikeda-Castrillon, S.K. \& Nunes, J.R.S. 2020c. Bird community structure in macrohabitats of the aquaticterrestrial transition zone in the Pantanal wetland, Brazil. Oecologia Australis, 24(3): 615-634. D0I

Gimenes, M.R.; Lopes, E.V.; Loures-Ribeiro, A.; Mendonça, L.B. \& Anjos, L. 2007. Aves da planície alagável do alto rio Paraná. Maringá, Editora da Universidade Estadual de Maringá. 281p.

Giraudo, A.R.; Chatellenaz, M.L.; Saibene, C.A.; Ordano, M.A.; Krauczuk, E.R.; Alonso, J. \& Di Giacomo, A.S. 2003. Avifauna del Iberá: composicion y datos sobre su historia natural. In: Alvarez, B.B. (Ed.). Fauna del Iberá. Buenos Aires, Editorial de la Universidad Nacional del Nordeste, Talleres Gráficos Volpe/Fox. p. 195-207.

Gräbin, D.M.; Tomas, M.A. \& Tomas, W.M. 2012. Densidade de Rhea americana em três paisagens diferentes do Pantanal da Nhecolândia, MS. Oecologia Australis, 16(4): 905-913.

Guedes, N.M.R. 1993. Biologia reprodutiva da arara-azul (Anodorhynchus hyacinthinus) no Pantanal - MS, Brasil. Piracicaba, Escola Superior de Agricultura "Luiz de Queiroz" - ESALQ/Universidade de São Paulo, Programa de Pós-Graduação em Ciências Florestais. Dissertação de Mestrado. $122 \mathrm{pp}$.

Guedes, N.M.R. \& Harper, L.H. 1995. The Hyacinth Macaw in the Pantanal. In: Abramson, J.; Speer, B.L. \& Thomsen, J.B. (Eds.). The large macaws: their care, breeding and conservation. Fort Bragg, Raintree Pub. p. 394-421.

Gwynne, J.A.; Ridgely, R.S.; Tudor, G. \& Argel, M. 2010. Aves do Brasil: Pantanal \& Cerrado. São Paulo, Editora Horizonte. 322p.

Hamilton, S.K.; Sippel, S.J. \& Melack, J.M. 1996. Inundation patterns in the Pantanal wetland of South America determined from passive microwave remote sensing. Archives für Hydrobiologie, 137: 1-23.

Hancock, P.; Muller, M. \& Tyler, S.J. 2007. Inventory of birds of the Okavango Delta Ramsar Site. Babbler, 49: 3-29.

Handbook of the birds of the world \& BirdLife International. 2020. Handbook of the birds of the world and Birdlife International digital checklist of the birds of the world. Version 5. Available: http://datazone.birdlife.org/ userfiles/file/Species/Taxonomy/HBW-BirdLife Checklist v5 Dec20. zip. Access: 02/01/2021.

Harris, M.B.; Tomas, W.M.; Mourão, G.; Silva, G.J.; Guimarães, E.; Sonoda, F. \& Facchini, E. 2005. Challenges to safeguard the Pantanal wetlands, Brazil: threats and conservation initiatives. Conservation Biology, 19(3): 714-720.

Hasenclever, L.; Reiman, C.: Mourão, G.M. \& Campos, Z.M.S. 2004. Densidades, tamanho de grupo e reprodução de emas no Pantanal Sul. Boletim de Pesquisa \& Desenvolvimento, EMBRAPA-CPAP, 55: 1-17.

Instituto Brasileiro do Meio Ambiente e dos Recursos Naturais Renováveis (IBAMA). 2007. Biodiversidade do Cerrado e Pantanal: áreas e ações prioritárias para conservação. Série Biodiversidade 17. Brasília, Ministério do Meio Ambiente. 540p. Available: http://www.mma.gov.br/ estruturas/chm/ arquivos/cerrado pantanal.pdf. Access: 09/01/2021.

Instituto Chico Mendes de Conservação da Biodiversidade (ICMBio). 2014. Espécies Ameaçadas - Lista 2014. Available: http://www.icmbio.gov.br/ portal/biodiversidade/fauna-brasileira/lista-de-especies.html. Access: 28/09/2020.

Jesus, F. \& Lima, S.F. 2003. Plano de Manejo do Parque Nacional do Pantanal. Brasília, DF, Instituto Brasileiro do Meio Ambiente e dos Recursos Naturais Renováveis/The Nature Conservancy. 543p.

Junk, W.J.; Cunha, C.N.; Wantzen, K.M.; Petermann, P.; Strüssmann, C; Maeques, M.I. \& Adis, J. 2006. Biodiversity and its conservation in the Pantanal of Mato Grosso, Brazil. Aquatic Science, 68: 1-32.

Junk, W.J.; Piedade, M.T.F.; Lourival, R.; Wittann, F.; Kandus, P.; Lacerda, L.D.; Bozelli, R.L.; Esteves, F.A.; Nunes da Cunha, C.; Maltchik, L.; Schöngart, J.; Schaeffer-Novelli, Y. \& Agostinho, A.A. 2014. Brazilian wetlands: their definition, and classification for research, sustainable management, and protection. Aquatic Conservation: Marine And Freshwater Ecosystems, 24: $5-22$.

Kantek, D.L.Z. \& Onuma, S.S.M. 2013. Primeiro registro documentado da Gaivota-de-Franklin Leucophaeus pipixcan Wagler, 1831 para o bioma Pantanal, Brasil. Ornithologia, 6(1): 106-108.

Kawamoto, A.H.N.; Mancini, D.A.P.; Pereira, L.E.; Cianciarullo, A.M.; Cruz, A.S.; Dias, A.L.F.; Mendonça, R.M.Z.; Pinto, J.R. \& Durigon, E.L. 2005. Investigation of influenza in migration birds, the primordial reservoir and transmitters of influenza in Brazil. Brazilian Journal of Microbiology, 36: 88-93.

Lago-Paiva, C. \& Willis, E.0. 1994. New occurrences of Melanerpes cactorum D'Orbigny, 1840) (Aves, Picidae) in Brazilian territory. Biotemas, 7(1-2): 110-115.

LASA-UFRJ - Laboratório de Aplicações de Satélites Ambientais da Universidade Federal do Rio de Janeiro. 2021. Área queimada - Pantanal 2020. Available: https://lasa.ufrj.br/alarmes. Access: 02/08/2021.

Leuzinger, L. 2011. Taxéus - Listas de espécies - Fazenda Barranco Alto. Available: http://www.taxeus.com.br/lista/192. Access: 09/05/2013.

Libonati, R.; DaCamara, C.C.; Peres, L.F.; Carvalho, S. \& Garcia, L.C. 2020. Rescue Brazil's burning Pantanal wetlands. Nature, 588: 217-219.

Lopes, L.E.; Pinho, J.B.; Ortiz, A.; Evangelista, M.M.; Silveira, L.F; Schunck, F. \& Develey, P.F. 2016. Birds from Cáceres, Mato Grosso: the highest species richness ever recorded in a Brazilian non-forest region. Revista Brasileira de Ornitologia, 24(2): 137-167.

Marengo, J.A.; Cunha, A.P.; Cuartas, L.A.; Leal, K.R.D.; Broedel, E.; Seluchi, M.E.; Michelin, C.M.; Baião, C.F.P.; Ângulo, E.C.; Almeida, E.K.; Kazmierczak, M.L.; Mateus, N.P.A.; Silva, R.C. \& Bender, F. 2021. Extreme drought in the Brazilian Pantanal in 2019-2010: characterization, causes, and impacts. Frontiers in Water, 3. D0I

Melo, A.V. \& Teribeli, R. 2008. Registro documentado de águia-chilena Buteo (Geranoaetus) melanoleucus para o Mato Grosso do Sul. Atualidades Ornitológicas, 144: 10.

Melo, A.V.; Santos, E.; Nunes, A.P. \& Tomas, W.M. 2007. Registro documentado do gavião-asa-de-telha (Parabuteo unicinctus) para o Mato Grosso do Sul. Atualidades Ornitológicas, 135: 14.

Mestre, L.A.M. 2007. Registros das migrações de trinta-réis-boreal Sterna hirundo: análise das recuperações de indivíduos marcados na América do Norte (1928-2005) e Brasil (1983-2005). Ornithologia, 2(2): 81-87.

Mestre, L.A.M.; Roos, A.L. \& Nunes, M.F. 2010. Análise das recuperações no Brasil de aves anilhadas no exterior entre 1927 e 2006. Ornithologia, 4(1): 15-35.

Miranda, C.S.; Paranhos-Filho, A.C. \& Pott, A. 2017. Changes in vegetation cover of the Pantanal wetland detect by Vegetation Index: a strategy for conservation. Biota Neotropica, 18(1). D0I 
Mitsch, W.J.; Bernal, B.B. \& Hernandez, M.E. 2015. Ecosystem services of wetlands. International Journal of Biodiversity Science, Ecosystem Service \&Management, 11(1): 1-4.

Munn, C.A.; Thomsen, J.A. \& Yamashita, C. 1989. The Hyacinth Macaw. In: Chadler, W.J. (Ed.). Audubon Wildlife Report. New York, Academic Press. p. 404-419.

Nabuco, H.C.G.; Castro, V.G.; Zuccheto, M.; Ferreira, T.V. \& Pinho, J.B. 2018. Notas Curtas: Comportamento de predação de Crypturellus undulatus (Tinamiformes: Tinamidae) por Accipiter poliogaster (Accipitriformes: Accipitridae) no Pantanal de Poconé, Mato Grosso, Brasil. Atualidades Ornitológicas, 202: 24.

Naumburg, E.M.B. 1935. Gazetteer and maps showing collecting stations visited by Emil Kaempfer in eastern Brazil and Paraguay. Bulletin of the American Museum of Natural History, 68: 449-469.

Naumburg, E.M.B.; Cherrie, G. \& Smith, H. 1930. The Birds of Mato Grosso, Brazil: a report on the birds secured by the Roosevelt-Rondon expedition. Bulletin of the American Museum of Natural History, 60: 1-431.

Nunes da Cunha, C. \& Junk, W.J. 2004. Year-to-year changes in water level drive the invasion of Vochysia divergens in Pantanal grasslands. Applied Vegetation Science, 7: 103-110.

Nunes, A.P. 2008. Distribuição de rei-do-bosque (Pheucticus aureoventris, (ardinalidae) no Brasil: revisão dos registros históricos e recentes. Atualidades Ornitológicas, 142: 38-40.

Nunes, A.P. 2009. Estado de conservação da avifauna ameaçada de extinção ocorrente no Pantanal, Brasil. Atualidades Ornitológicas, 157: 85-98.

Nunes, A.P. 2011a. Quantas espécies de aves ocorrem no Pantanal brasileiro? Atualidades Ornitológicas, 160: 45-54.

Nunes, A.P. 2011b. Registros Ornitológicos na região alagada pelo Rio Taquari, Mato Grosso do Sul, Brasil. Atualidades Ornitológicas, 160: 38-44.

Nunes, A.P. 2015. Efeito da heterogeneidade da paisagem e do habitat na comunidade de aves no oeste do Pantanal da Nhecolândia, Mato Grosso do Sul. Campo Grande, Universidade Federal de Mato Grosso do Sul, Programa de Pós-Graduação em Ecologia e Conservação. Tese de Doutorado. 263p.

Nunes, A.P. \& Tomas, W.M. 2004a. Análise preliminar das relações biogeográficas da avifauna do Pantanal com biomas adjacentes. In: Simpósio sobre Recursos Naturais e Sócio-econômicos do Pantanal, $4^{\circ}$. Anais. Corumbá, EMBRAPA Pantanal. p. 1-8.

Nunes, A.P. \& Tomas, W.M. 2004b. Aves migratórias ocorrentes no Pantanal: caracterização e conservação. Série Documentos, EMBRAPA-CPAP, 62: 1-27.

Nunes, A.P. \& Tomas, W.M. 2008. Aves migratórias e nômades ocorrentes no Pantanal. Corumbá, EMBRAPA-CPAP.

Nunes, A.P.; Godoi, M.N.; Pivatto, M.A.C.; Morante-Filho, J.C.; Patrial, E.W.; Silva, P.A.; Stavis, V.K.; Manço, D.D.G.; Costacurta, M.B.; Leuchtenberger, C. \& Lehn, C.R. 2013. Aves da Serra de Maracaju, Mato Grosso do Sul, Brasil. Revista Brasileira de Ornitologia, 21(1): 75-100.

Nunes, A.P.; Silva, P.A. \& Tomas, W.M. 2008. Novos registros de aves para 0 Pantanal, Brasil. Revista Brasileira de Ornitologia, 16(2): 160-164.

Nunes, A.P.; Tizianel, F.A.T.; Leuchtenberger, C.; Navarro, T.A.M.; Melo, A.V. \& Campis, M.C. 2011. Registros documentados da ocorrência de saracuracarijó (Pardirallus maculatus, Rallidae) no Pantanal e estado de Mato Grosso do Sul, Brasil. Atualidades Ornitológicas, 164: 8-10.

Nunes, A.P.; Tizianel, F.A.T.; Melo, A.V.; Nascimento, V. \& Machado, N. 2010. Aves da Estrada Parque Pantanal, Corumbá, Mato Grosso do Sul, Brasil. Atualidades Ornitológicas, 156: 33-47.

Nunes, A.P.; Tizianeli, F.A.T.; Tomas, W.M. \& Lupinetti, C. 2009. Aves da fazenda Nhumirim e seus arredores: Lista 2008. Boletim de Pesquisa e Desenvolvimento, EMBRAPA-CPAP, 89: 1-44.

Nunes, A.P.; Vasconcelos, M.F.; Hoffmann, D.; Souza, L.N.; Gomes, C.R.G.; Epifânio, A.D.; Godoi, M.N.; Tambelini, F.A.T.; Straube, F.C.; Silva, P.A.;
Posso, S.R.; Laps, R.R.; Faria, S.P. \& Tomas, W.M. 2018. Aves da borda oeste do Pantanal, Mato Grosso do Sul, Brasil. Atualidades Ornitológicas, 206: 47-69.

Nunes, J.R.S. 2010. Avifauna do Rio Paraguai, Pantanal de Cáceres, Mato Grosso. São Carlos, Universidade Federal de São Carlos, Programa de PósGraduação em Ecologia e Recursos Naturais. Tese de Doutorado. 256 pp.

Oliveira, D.M.M. 1997. Sucesso reprodutivo e conservação de tuiuiú Jabiru mycteria (Aves: Ciconiidae) no Pantanal de Poconé, Mato Grosso. Cuiabá, Universidade Federal do Mato Grosso, Programa de Pós-Graduação em Ecologia e Conservação da Biodiversidade. Dissertação de Mestrado. $68 \mathrm{pp}$.

Oliveira, D.M.M. 2006. Efeitos bióticos e abióticos de ambientes alagáveis nas assembleias de aves aquáticas e piscívoras no Pantanal, Brasil. Manaus, Instituto Nacional de Pesquisas da Amazônia, Programa de PósGraduação em Ecologia. Tese de Doutorado. 198p.

Pacheco, J.F. \& Bauer, CA 1994. A coleção de aves preparadas por Adolf Schneider em Porto Quebracho, Mato Grosso do Sul, Brasil, em 1941. Nótulas Faunísticas, 64: 1-6.

Pereira, M.S. \& Poerschke, F. 2010. New birds records from Lagoa do Peixe National Park, southern Brazil. Biotemas, 23(1): 241-246.

Petermann, P. 2010. Birds. In: Junk, W.J.; Da Silva, C.J.; Cunha, C.N. \& Wantzen, K.M. (Eds.). The Pantanal: Ecology, biodiversity and sustainable management of a large neotropical seasonal wetland. Sofia, Pensoft Publishers. p. 523-562.

Pinho, J.B. 1998. Aspectos comportamentais da arara azul (Anodorhynchus hyacinthinus) na localidade de Pirizal, Município de Nossa Senhora do Livramento, Pantanal de Poconé. Cuiabá, Universidade Federal de Mato Grosso, Programa de Pós-Graduação em Ecologia e Conservação da Biodiversidade. Dissertação de Mestrado. 78p.

Pinho, J.B. 2005. Riqueza de espécies, padrão de migração e biologia reprodutiva de aves em quatro ambientes florestais do Pantanal de Poconé, MT. Belo Horizonte, Universidade Federal de Minas Gerais, Programa de Pós-Graduação em Ecologia, Conservação e Manejo de Vida Silvestre. $185 \mathrm{pp}$.

Pinto, M.M.; Libonati, R.; Trigo, R.M.; Trigo, I.F. \& DaCamara, C.C. 2020. A deep learning approach for mapping and dating burned areas using temporal sequences of satellite images. ISPRS Journal of Photogrammetry and Remote Sensing, 160: 260-274.

Pinto, 0.M.0. 1932. Resultados ornithológicos de uma excursão pelo Oeste de São Paulo e Sul de Matto Grosso. Revista do Museu Paulista, 17(2): 689-826.

Pinto, 0.M.0. 1938. Catálogo das aves do Brasil e lista dos exemplares que

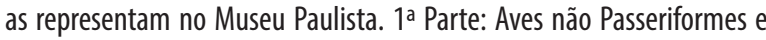
Passeriformes não Oscines, excluída a família Tyrannidae e seguintes. Revista do Museu Paulista, 22: 1-566.

Pinto, 0.M.0. 1940. Nova contribuição à ornitologia de Mato Grosso. Arquivo de Zoologia, 2(1): 1-37.

Pinto, 0.M.0. 1944. Catálogo das aves do Brasil, 2a parte. Ordem Passeriformes (continuação): superfamília Tyrannoidea e Subordem Passeres. São Paulo, Secretaria de Agricultura de São Paulo. 700 pp.

Pinto, 0.M.0. 1948. Notas e impressões naturalísticas de uma viagem fluvial a Cuiabá. Boletim Museu Paraense Emílio Goeldi, 10: 331-354.

Pivatto, M.A.C. \& Bernardon, G. 2012. Guia Fotográfico - Aves do Pantanal. São Paulo, Aves \& Fotos Editora. 256p.

Pivatto, M.A.C.; Donatelli, R.J. \& Manço, D.D.M. 2008. Aves da fazenda Santa Emília, Aquidauana, Mato Grosso do Sul. Atualidades Ornitológicas, 143: 33-37.

Prado, D.E. 1993a. What is the Gran Chaco vegetation in South America? I. A review. Constribution to the study of flora and vegetation of the Chaco. V. Candollea, 48(1): 145-172. 
Prado, D.E. 1993b. What is the Gran Chaco vegetation in South America? I. A redefinition. Constribution to the study of flora and vegetation of the Chaco. VII. Candollea, 48(2): 615-629.

Prado, D.E. \& Gibbs, P.E. 1993. Patterns of species distributions in the dry seasonal forest South America. Annals of the Missouri Botanic Garden, 80(4): 902-927.

Ratter, J.A.; Bridgewater, J.F. \& Ribeiro, J.F. 2003. Analysis of the floristic composition of the Brazilian cerrado vegetation III: comparison of the woody vegetation of 376 areas. Edinburgh Journal Botany, 60: 57-109.

Reichholf, J. 1976. Camp fires and abundance of birds nest in the Southern Mato Grosso Cerrados and Pantanal, Brazil. Journal für Ornithologie, 117: 464-465.

Ribas, C.; Manço, D.D.G.; Pivatto, M.A.C.; Copetti, M.; Copetti, L.; Zapparoli, C. \& Melo, F. 2001. Bird Watching - Refúgio da Ilha Pousada e Ecologia. Miranda, Refúgio da llha Pousada e Ecologia. 40p.

Ruschi, A. 1955. Algumas observações sobre a Trochilifauna da região do Pantanal Matogrossense, compreendida entre Cáceres e Tapirapoan, ou seja, entre o Pantanal Norte e encosta dos Parecis. Boletim Museu Biologia Prof. Mello Leitão, 17(1): 1-19.

Salvadori, T. 1895. Uccelli raccolti nel Paraguai, nel Mato Grosso, nel Tucumã e nella Provincia di Salta. Bolletino dei Musei di Zoologia ed Anatomia Comparada della Reale Università di Torino, 10(208): 1-24.

Salvadori, T. 1900. Viaggio del Dr. A. Borelli nel Mato Grosso e nel Paraguai. V. Uccelli. Bolletino dei Musei di Zoologia ed Anatomia Comparada della Reale Università di Torino, 15: 1-19.

Schubart, 0.; Aguirre, A.C. \& Sick, H. 1965. Contribuição para o conhecimento da alimentação das aves brasileiras. Arquivos de Zoologia, São Paulo, 12: 95-249.

Serrano, I.L. 2010. Distribuição e conservação de aves migratórias neárticas da Ordem Charadiiformes (familias Charadriidae e Scolopacidae) no Brasil. Belém, Museu Paraense Emílio Goeldi/Universidade Federal do Pará, Programa de Pós-Graduação em Zoologia. Tese de Doutorado. 174p.

Severo-Neto, F.; Leuzinger, L. \& Faria, S.P. 2017. Oxyura vittata (Philippi, 1860) (Aves, Anatidae): range extension and first record from the Upper Paraguay basin, Brazil. Check List, 13(4): 285-287.

Severo-Neto, F.; Melo, A.V. \& Souza, F.L. 2015. New records of Sporophila nigrorufa (D'0rbigny \& Lafresnaye, 1837) in Brazilian Cerrado and Pantanal. Revista Brasileira de Ornitologia, 23(3): 357-359.

Sick, H. 1961. Die Spechte Trichopicus cactorum und Scapaneus leucopogon in Brasilien. Journal of Ornithology, 102(4): 401-403.

Sick, H. 1997. Ornitologia Brasileira. Rio de Janeiro, Nova Fronteira. 862p.

Signor, C.A. \& Pinho, J.B. 2010. Aves. In: Fernandes, I.M.; Signor, C.A. \& Penha, J. (Orgs.). Biodoversidade no Pantanal de Poconé. Cuiabá, Centro de Pesquisas do Pantanal. p. 137-154.

Silva, J.M.C. 1995. Birds of the Cerrado region, South America. Steenstrupia, 21: 69-92.

Silva, J.M.C. 1996. Distribuition of Amazonian and atlantic birds in gallery forest of the cerrado region, south America. Ornitologia Neotropica, 7(1): 1-18.

Silva, J.M.C. \& Bates, J.M. 2002. Biogeographic patterns and conservation in the South American Cerrado: a tropical savanna hotspot. BioScience, 52(3): 225-233.

Silva, J.M.C. \& Santos, M.P.D. 2005. A importância relativa dos processos biogeográficos na formação da avifauna do Cerrado e de outros biomas brasileiros. In:Scariot, A.; Sousa-Silva, J.C. \& Felfili, J.M. Cerrado: ecologia, biodiversidade e conservação. Brasília, DF, Ministério do Meio Ambiente. p. 224-233.

Silveira, L.F. \& Straube, F.C. 2008. Aves ameaçadas de extinção no Brasil. In: [p. 379-666]. Machado, A.B.M.; Drummond, G.M. \& Paglia, A.P. (Eds.). Livro Vermelho da Fauna Brasileira Ameaçada de Extinção. Brasília, Ministério do Meio Ambiente/Fundação Biodiversitas. v. 2, 1420p.
Somenzari, M.; Amaral, P.P. do, Cueto, V.R.; Guaraldo, A.D.C.; Jahn, A.E.; Lima, D.M.; Lima, P.C.; Lugarini, C.; Machado, C.G.; Martinez, J.; Nascimento, J.L.X. do; Pacheco, J.F.; Paludo, D.; Prestes, N.P.; Serafini, P.P.; Silveira, L.F.; Sousa, A.E.B.A. de; Sousa, N.A. de; Souza, M.A. de; Telino-Junior, W.R. \& Whitney, B.M. 2018. An overview of migratory birds in Brazil. Papéis Avulsos de Zoologia, 58: 1-66. D0I

Souza, D. 2005. Um exemplar esquecido do jacupixuna Penelope obscura, oriundo de Mato Grosso do Sul. Atualidades Ornitológicas, 128: 11.

Souza-Jr., C.M.; Shimbo, J.Z.; Rosa, M.R.; Parente, L.L.; Alencar, A.A.; Rudorff, B.F.T.; Hasenack, H.; Matsumoto, M.; Ferreira, L.G.; Souza-Filho, P.W.M.; Oliveira, S.W.; Rocha, W.F.; Fonseca, A.V.; Marques, C.B.; Diniz, C.G.; Costa, D.; Monteiro, D.; Rosa, E.E.; Vélez-Martin, E.; Weber, E.J.; Lenti, F.E.B.; Paternost, F.E.; Pareyn, F.G.C.; Siqueira, J.V.; Vieira, J.L.; Ferreira-Neto, L.C.; Saraiva, M.M.; Sales, M.H.; Salgado, M.P.G.; Vasconcelos, R.; Galano, S.; Mesquita, V.V. \& Azevedo, T. 2020. Reconstructing three decades of land use and land cover changes in brazilian biomes with landsat archive and earth engine. Remote Sensing, 12(17). DOI

Stone, W. \& Roberts, H.R. 1934. Zoological results of the Mato Grosso expedition to Brasil in 1931 - Birds. Proceedings of the Academy of Natural Sciences of Philadelphia, 86: 363-397.

Straube, F.C. 2010. As viagens de Alfredo Borelli (1893-1899) ao Brasil com notas biográficas e revisão ornitológica. Atualidades Ornitológicas, 155: 49-55.

Straube, F.C. \& Melo, F. 2011. Refúgio Ecológico Caiman. In: Valente, R.M.; Silva, J.M.C.; Straube, F.C. \& Nascimento, J.L.X. (Eds.). Conservação de aves migratórias neárticas no Brasil. Belém, Conservação Internacional. p. 201-214.

Straube, F.C.; Urben-Filho, A.; Deconto, L.R. \& Patrial, E.W. 2007. Fluvicola nengeta (Linnaeus, 1766) nos estados do Paraná e Mato Grosso do Sul e sua expansão de distribuição geográfica pelo sul do Brasil. Atualidades Ornitológicas, 137: 33-38.

Straube, F.C.; Urben-Filho, A.; Pivatto, M.A.C.; Nunes, A.P. \& Tomas, W.M. 2006a. Nova contribuição à ornitologia do Chaco Brasileiro (Mato Grosso do Sul, Brasil). Atualidades Ornitológicas, 134: 1-27.

Straube, F.C.; Urben-Filho, A.; Nunes, A.P.; Tomas, W.M. \& Vieira-da-Rocha, M.C. 2006b. Avifauna do Pantanal do Nabileque (Mato Grosso do Sul, Brasil). Atualidades Ornitológicas, 134: 1-22.

Strüssmann, C. 1998. Presence of the White-fronted woodpecker Melanerpes cactorum (Piciformes: Picidae) in the Northern Pantanal, Mato Grosso State, Brazil. Revista Biologia Tropical, 46(4): 1199.

Timothy, J.K.; Chavez, E.; Peña-Claros, M.; Toledo, M.; Arroyo, L.; Caballero, J.; Correa, L.; Guillén, R.; Quevedo, R.; Saldias, M.; Soria, L.; Uslar, Y.; Vargas, I. \& Steininger, M. 2006. The Chiquitano dry forest, the transition between Humid and Dry Forest in Eastern Lowland Bolivia. In: Pennington, R.T.; Lewis, G.P. \& Ratter, J.A. (Eds.). Neotropical Savannas and seasonally dry forests: plant diversity, biogeography and conservation. London, Taylor \& Francis. p. 213-233.

Tomas, W.M.; Mourão, G.M.; Campos, Z.M.S.; Salis, S.M. \& Santos, S.A. 2009. Intervenções humanas na paisagem e nos habitats do Pantanal. Corumbá, EMBRAPA-CPAP.

Tomas, W.M.; Nunes, A.P.; Cáceres, N.; Fisher, E.; Campos, Z.; Aragona, M.; Mourão, G.; Antunes, P. 2008. Mammals and birds from Pantanal and Upper Paraguay River Basin in Brazil, Bolivia and Paraguay. In: INTECOL - International Wetlands Conference, $8^{\circ}$. Anais. Cuiabá, International Association for Ecology/Universidade Federal de Mato Grosso/Centro de Pesquisas do Pantanal. p. 228.

Travassos, L. 1940. Relatório da terceira excursão à zona da Estrada de Ferro Noroeste do Brasil realizada em fevereiro e março de 1940. Memórias do Instituto Oswaldo Cruz, 35(3): 607-696.

Travassos, L. 1941. Relatório da quinta excursão à zona da Estrada de Ferro Noroeste do Brasil realizada em janeiro de 1941. Memórias do Instituto Oswaldo Cruz, 36(3): 263-300. 
Travassos, L. \& Freitas, J.F.T. 1940. Relatório da excursão científica realizada na zona da Estrada de Ferro Noroeste do Brasil em julho de 1939, Memórias do Instituto Oswaldo Cruz, 35(3): 525-556.

Travassos, L. \& Freitas, J.F.T. 1942. Relatório da sexta excursão do Instituto Oswaldo Cruz, realizada à zona da Estrada de Ferro Noroeste do Brasil, em Novembro de 1941. Memórias do Instituto Oswaldo Cruz, 37(3): 259-286.

Travassos, L.; Travassos, H.; Rego-Barros, A.R.; Albuquerque, D.0.; Oliveira, S.J.; Castro, A.L. \& Lopes, H.S. 1957. Excursão científica realizada nas zonas das Estradas de Ferro Noroeste do Brasil e Brasil-Bolívia em janeiro e fevereiro de 1955. Publicação Avulsas Museu Nacional do Rio Janeiro, 20(1): 1-19.

Tubelis, D.P. \& Tomas, W.M. 1999. Distribution of birds in a naturally patchy forest environment in the Pantanal wetland, Brazil. Ararajuba, 7(2): 81-89.

Tubelis, D.P. \& Tomas, W.M. 2003a. Bird species of the Pantanal wetland, Brazil. Ararajuba, 11(1): 5-37.

Tubelis, D.P. \& Tomas, W.M. 2003b. The contributions of museum collection and of records not involving collections to the knowledge bird species composition of the Pantanal, Brazil. Ararajuba, 11(2): 207-214.

Ubaid, F.K. \& Antas, P.T.Z. 2013. Novos registros de aves para a Reserva Particular do Patrimônio Natural SESC Pantanal, Barão de Melgaço, MT. Ornithologia, 5(2): 122-130.

Ubaid, F.K. \& Donatelli, R.J. 2008. Primeiro registro documentado da garçada-mata (Agamia agami, Ardeidae) para o Estado do Mato Grosso do Sul, Brasil. Atualidades Ornitológicas, 142: 44-45.

Ubaid, F.K.; Ferreira, L.P.; Oliveira-Júnior, S.B. \& Antas, P.T.Z. 2010. Primeiro registro de Harpia harpyja para o bioma Pantanal, com dados sobre atividade reprodutiva. Revista Brasileira de Ornitologia, 19(1): 88-92.

Vasconcelos, M.F. \& Hoffmann, D. 2006. Os Bosques Secos Chiquitanos também são nossos! Atualidades Ornitológicas, 130: 10-11.

Vasconcelos, M.F.; Lopes, L.E.; Hoffmann, D.; Silveira, L.F. \& Schunck, F. 2008. Noteworthy records of birds from the Pantanal, Chiquitano dry forest and Cerrado of south-western Brazil. Bulletin British Ornthologist Club, 128(1): 57-67.

VertNet - National Science Foundation: where discoveries begin. 2021. Distributed databases with backbone. Available: http://portal.vertnet. org/search. Access: 15/03/2021.
Visual Resources for Ornithology (VIRE0). 2021. Birds fotos and images of birds worldwide. The Academy of Natural Sciences of Drexel University. Available: http://vireo.ansp.org. Access: 22/03/2021.

Vitorino, D.B.; Frota, A.V.B.; Ângelo, M. \& Nunes, J.R.S. 2017. Avifauna associada a duas áeas de nascentes no Assentamento Laranjeira I, Província Serrana, Cáceres, MT. In: Castrillon, S.I.; Puhl, J.I.; Morais, F.F. \& Lopes, A.A.E.T.M. (Orgs.). Escassez hídrica e restauração ecológica no Pantanal: recuperação das nascente e fragmentos de mata ciliar do córrego no Assentamento Laranjeira I e mobilização para conservação dos recursos hídricos no Pantanal mato-grossense. Cuiabá, Carlini \& Caniato Editorial. p. 153-167.

Weinberg, L.F. 1984. Aves do Pantanal do Mato Grosso do Sul. Boletim FBCN, Rio de Janeiro, 19(1): 81-88.

Werneck, F.P.; Costa, G.C.; Colli, G.R.; Prado, D.E. \& Sites-Jr., J.W. 2011. Revisiting the historical distribution of Seasonally Dry Tropical Forests: new insights based on palaeodistribution modelling and palynological evidence. Global Ecology and Biogeography, 20: 272-288.

Whittaker, A.; Zimmer, K.J. \& Carlos, B. 2008. The status of Mississippi Kite Ictinia mississippiensis in Brazil, including further documented records for the country. Cotinga, 29: 139-143.

WikiAves - A Enciclopédia das Aves do Brasil. 2021. Available: http://www. wikiaves.com. Access: 06/03/2021.

Willis, E.0. 1995. Black versus white waterbird colonies (Aves) in the BolivianBrazilian Pantanal. Iheringia, Série Zoologia, 78(1): 95-97.

Willis, E.0. \& Oniki, Y. 1990. Levantamento preliminar das aves de inverno em dez áreas do sudoeste de Mato Grosso, Brasil. Ararajuba, 1(1): 19-38.

Xeno-Canto Foundation - Sharing bird sounds from around the world. 2021. Available: http://www.xeno-canto.org. Access: 09/03/2021.

Yabe, R.S.; Marques, E.J. \& Marini, M.Â. 2010. Movements of birds among natural vegetation patches in the Pantanal, Brazil. Bird Conservation International, 20: 400-409.

Yamashita, C. 1997. Anodorhynchus macaws as followers of extinct megafauna: an hypothesis. Ararajuba, 5(2): 176-182.

Yamashita, C. \& Valle, M.P. 1990. Sobre ninhais de aves do Pantanal do Município de Poconé, Mato Grosso, Brasil. Vida Silvestre Neotropical, 2(2): 59-63.

Zimmer, J.T. 1933. Studies of Peruvian birds. X. The Formicarian genus Thamnophilus. Part 2. American Museum Novitates, 647: 1-27. 


\section{SUPPLEMENTARY MATERIAL}

Sites and regions with records of birds species in the Pantanal wetland, followed by their geographic coordinates and their sources of information.

\begin{tabular}{|c|c|c|}
\hline Localities & Coordinates & Reference \\
\hline Albuquerque & $19^{\circ} 24^{\prime} S, 57^{\circ} 24^{\prime} 0$ & Tubelis \& Tomas (2003a), Vasconcelos et al. (2008) \\
\hline APA Baía Negra & $19^{\circ} 01^{\prime} S, 57^{\circ} 31^{\prime} 0$ & This study (GOF \& RADS, 2018 and 2019) \\
\hline Aquidauana & $20^{\circ} 29^{\prime} S, 55^{\circ} 48^{\prime} 0$ & Tubelis \& Tomas (2003a), Whittaker et al. (2008) \\
\hline Área próxima ao Rio Vermelho & $19^{\circ} 36^{\prime} S, 56^{\circ} 51^{\prime} 0$ & Tubelis \& Tomas (2003a) \\
\hline Área urbana de Porto Murtinho & $21^{\circ} 41^{\prime} S, 57^{\circ} 52^{\prime} 0$ & Benites et al. (2017) \\
\hline Assentamento Laranjeiras & $16^{\circ} 34^{\prime} S, 57^{\circ} 32^{\prime} 0$ & Vitorino et al. (2017), this study (BDV \& AVBF, 2016) \\
\hline Baía do Malheiros & $16^{\circ} 03^{\prime} S, 57^{\circ} 41^{\prime} 0$ & Nunes (2010), this study (AVBF \& BDV, January 2019 and January 2020) \\
\hline Baía do Retiro Velho & $16^{\circ} 10^{\prime} S, 57^{\circ} 46^{\prime} 0$ & Nunes (2010) \\
\hline Baía Boca do Natalino & $16^{\circ} 30^{\prime} S, 57^{\circ} 47^{\prime} 0$ & Nunes (2010) \\
\hline Baía da Guaíva & $16^{\circ} 39^{\prime} S, 57^{\circ} 10^{\prime} 0$ & Tubelis \& Tomas (2003a) \\
\hline Baía do Morro & $16^{\circ} 42^{\prime} S, 57^{\circ} 46^{\prime} 0$ & Nunes (2010) \\
\hline Baía Negra & $16^{\circ} 48^{\prime} S, 57^{\circ} 39^{\prime} 0$ & Kantek \& Onuma (2013) \\
\hline Baía Simão Nunes & $16^{\circ} 19^{\prime} S, 57^{\circ} 44^{\prime} 0$ & Nunes (2010) \\
\hline Base de Estudos do Pantanal & $19^{\circ} 34^{\prime} \mathrm{S}, 57^{\circ} 01^{\prime} 0$ & This study (SRP \& RRL, 2011 and 2020) \\
\hline Base do IBAMA, Transpantaneira & $17^{\circ} 12^{\prime} S, 57^{\circ} 00^{\prime} 0$ & Tubelis \& Tomas (2003a) \\
\hline Boca de Hormiguera & $19^{\circ} 03^{\prime} S, 57^{\circ} 19^{\prime} 0$ & Tubelis \& Tomas (2003a) \\
\hline Cáceres & $16^{\circ} 08^{\prime} S, 57^{\circ} 43^{\prime} 0$ & Tubelis \& Tomas (2003a), Lopes et al. (2016), WikiAves (2021) \\
\hline Caiçara & $16^{\circ} 04^{\prime} S, 57^{\circ} 45^{\prime} 0$ & Tubelis \& Tomas (2003a), Lopes et al. (2016) \\
\hline Flood área in BR-262 & $19^{\circ} 34^{\prime} S, 57^{\circ} 10^{\prime} 0$ & This study (APN, July 2011) \\
\hline Cambará & $16^{\circ} 33^{\prime} S, 57^{\circ} 51^{\prime} 0$ & Lopes et al. (2016) \\
\hline Campo Florido & $21^{\circ} 39^{\prime} S, 57^{\circ} 43^{\prime} 0$ & This study (December 2013 and February 2014) \\
\hline Campus da UFMS/Corumbá & $18^{\circ} 59^{\prime} S, 57^{\circ} 37^{\prime} 0$ & Nunes et al. (2011) \\
\hline Carandazal/MS-325 & $19^{\circ} 43^{\prime} S, 57^{\circ} 04^{\prime} 0$ & This study (November 2011) \\
\hline Carandazinho & $18^{\circ} 39^{\prime} S, 57^{\circ} 32^{\prime} 0$ & Tubelis \& Tomas (2003a) \\
\hline Centro de Pesquisa da Vida Selvagem do Pantanal & $16^{\circ} 07^{\prime} S, 56^{\circ} 59^{\prime} 0$ & WikiAves (2021) \\
\hline Chácara Aromita & $21^{\circ} 42^{\prime} S, 57^{\circ} 51^{\prime} 0$ & Benites \& Mamede (2021), this study (MB \& SM, 2020) \\
\hline Chácara Brasília & $21^{\circ} 42^{\prime} S, 57^{\circ} 50^{\prime} 0$ & Benites \& Mamede (2021), this study (MB \& SM, 2020) \\
\hline Corumbá & $18^{\circ} 59^{\prime} S, 57^{\circ} 38^{\prime} 0$ & Tubelis \& Tomas (2003a), Mestre (2007), Nunes et al. (2008), Mestre et al. (2010), this study (APN, 2004 to 2013) \\
\hline Curva do Leque & $19^{\circ} 15^{\prime} S, 57^{\circ} 03^{\prime} 0$ & Nunes et al. (2010), Serrano (2010), WikiAves (2021) \\
\hline Descalvados/Fedegoso & $16^{\circ} 43^{\prime} S, 57^{\circ} 45^{\prime} 0$ & Tubelis \& Tomas (2003a), Lopes et al. (2016) \\
\hline Military base of Porto Murtinho & $21^{\circ} 05^{\prime} \mathrm{S}, 57^{\circ} 50^{\prime} 0$ & This study (APN, November 2011) \\
\hline Dique de contenção de inundação e área rural de Porto Murtinho & $21^{\circ} 41^{\prime} S, 57^{\circ} 52^{\prime} 0$ & Benites et al. (2017), Benites \& Mamede (2021) \\
\hline Estação Barranco Branco & $21^{\circ} 05^{\prime} \mathrm{S}, 57^{\circ} 50^{\prime} 0$ & Benites et al. (2017) \\
\hline Estação Ecológica de Taiamã & $16^{\circ} 52^{\prime} S, 57^{\circ} 280^{\prime} 0$ & Lopes et al. (2016), Frota et al. (2020a) \\
\hline Estação Ingazeira & $22^{\circ} 04^{\prime} S, 57^{\circ} 56^{\prime} 0$ & Benites et al. (2017), Benites \& Mamede (2021) \\
\hline Fazenda Acurizal & $17^{\circ} 49^{\prime} S, 57^{\circ} 32^{\prime} 0$ & Allen (1891), Benites \& Mamede (2021), this study (FCS, June 2003; SRP \& RRL, September 2014) \\
\hline Fazenda Aguapé & $20^{\circ} 06^{\prime} S, 55^{\circ} 57^{\prime} 0$ & Tubelis \& Tomas (2003a), WikiAves (2021), Xeno-Canto (2021) \\
\hline Fazenda Alegria & $19^{\circ} 03^{\prime} S, 56^{\circ} 47^{\prime} 0$ & Tubelis \& Tomas (2003a), Nunes et al. (2009), Nunes (2015) \\
\hline Fazenda Alvorada & $17^{\circ} 16^{\prime} S, 56^{\circ} 15^{\prime} 0$ & This study (WMT, 2007) \\
\hline Fazenda Baía, Pixaim & $16^{\circ} 40^{\prime} S, 56^{\circ} 48^{\prime} 0$ & Tubelis \& Tomas (2003a) \\
\hline Fazenda Baía Bonita & $18^{\circ} 40^{\prime} S, 56^{\circ} 26^{\prime} 0$ & Tubelis \& Tomas (2003a) \\
\hline Fazenda Baía das Pedras & $19^{\circ} 15^{\prime} \mathrm{S}, 55^{\circ} 46^{\prime} 0$ & This study (RJD, November 2007) \\
\hline Fazenda Baía de Pedra & $16^{\circ} 28^{\prime} S, 58^{\circ} 08^{\prime} 0$ & Lopes et al. (2016) \\
\hline Fazenda Baía Grande & $20^{\circ} 20^{\prime} S, 56^{\circ} 15^{\prime} 0$ & WikiAves (2021) \\
\hline Fazenda Barra Mansa & $19^{\circ} 35^{\prime} \mathrm{S}, 56^{\circ} 05^{\prime} 0$ & Tubelis \& Tomas (2003a) \\
\hline Fazenda Barranco Alto/Salina & $19^{\circ} 35^{\prime} S, 56^{\circ} 09^{\prime} 0$ & Tubelis \& Tomas (2003a), Leuzinger (2011) \\
\hline Fazenda Barranco Branco & $21^{\circ} 05^{\prime} \mathrm{S}, 57^{\circ} 50^{\prime} 0$ & This study (APN, November 2011) \\
\hline Fazenda Bela Vista & $19^{\circ} 14^{\prime} S, 57^{\circ} 26^{\prime} 0$ & Nunes et al. $(2008,2010)$ \\
\hline Fazenda Braunal & $22^{\circ} 06^{\prime} \mathrm{S}, 57^{\circ} 44^{\prime} 0$ & Straube et al. (2006a), this study (APN, November 2011) \\
\hline Fazenda Caiman & $19^{\circ} 56^{\prime} \mathrm{S}, 56^{\circ} 20^{\prime} 0$ & Tubelis \& Tomas (2003a), Nunes (2011a), Straube \& Melo (2011), WikiAves (2021), Xeno-Canto (2021) \\
\hline Fazenda Caité & $18^{\circ} 43^{\prime} S, 55^{\circ} 15^{\prime} 0$ & Nunes et al. (2008), this study (APN, May 2005) \\
\hline Fazenda Campo Lourdes & $19^{\circ} 32^{\prime} \mathrm{S}, 55^{\circ} 38^{\prime} 0$ & This study (JRD, July 2007) \\
\hline Fazenda Campo Novo & $19^{\circ} 22^{\prime} S, 57^{\circ} 37^{\prime} 0$ & Nunes et al. (2008) \\
\hline Fazenda Cerro Porã & $22^{\circ} 01^{\prime} S, 57^{\circ} 31^{\prime} 0$ & Benites et al. (2017) \\
\hline
\end{tabular}




\begin{tabular}{|c|c|c|}
\hline Localities & Coordinates & Reference \\
\hline Pousada das Araras & $16^{\circ} 30^{\prime} S, 56^{\circ} 42^{\prime} 0$ & Xeno-Canto (2021), WikiAves (2021) \\
\hline Fazenda Fazendinha & $19^{\circ} 29^{\prime} S, 56^{\circ} 29^{\prime} 0$ & Tubelis \& Tomas (2003a) \\
\hline Fazenda Figueirinha & $19^{\circ} 15^{\prime} \mathrm{S}, 57^{\circ} 40^{\prime} 0$ & Vasconcelos et al. (2008) \\
\hline Fazenda Firme/Fazenda Leque & $19^{\circ} 15^{\prime} S, 57^{\circ} 01^{\prime} 0$ & Tubelis \& Tomas, 2003a), Serrano (2010), WikiAves (2021) \\
\hline Fazenda Jofre, Transpantaneira & $17^{\circ} 17^{\prime} \mathrm{S}, 56^{\circ} 50^{\prime} 0$ & Tubelis \& Tomas (2003a) \\
\hline Fazenda Lucero Porã & $21^{\circ} 54^{\prime} S, 57^{\circ} 38^{\prime} 0$ & This study (APN, November 2011) \\
\hline Fazenda Monjolo & $19^{\circ} 19^{\prime} \mathrm{S}, 57^{\circ} 34^{\prime} 0$ & Vasconcelos et al. (2008) \\
\hline Fazenda Novos Dourados/RPPN Engenheiro Eliezer Batista & $18^{\circ} 05^{\prime} S, 57^{\circ} 28^{\prime} 0$ & This study (APN, January and June 2007; RRL, September 2012 and September 2013) \\
\hline Fazenda Nhumirim & $18^{\circ} 59^{\prime} S, 56^{\circ} 39^{\prime} 0$ & Tubelis \& Tomas (1999, 2003a), Chiaravalloti et al. (2009), Nunes et al. (2009), Nunes (2015) \\
\hline Fazenda Nova Esperança & $17^{\circ} 54^{\prime} S, 56^{\circ} 47^{\prime} 0$ & WikiAves (2021) \\
\hline Fazenda Pacu & $20^{\circ} 38^{\prime} S, 57^{\circ} 37^{\prime} 0$ & Straube et al. (2006b) \\
\hline Fazenda Palmeiras/Palmira & $18^{\circ} 55^{\prime} \mathrm{S}, 57^{\circ} 03^{\prime} 0$ & Tubelis \& Tomas (2003a) \\
\hline Fazenda Porto Conceição & $20^{\circ} 28^{\prime} S, 57^{\circ} 55^{\prime} 0$ & Straube et al. (2006a) \\
\hline Fazenda Paraputanga & $15^{\circ} 56^{\prime} \mathrm{S}, 57^{\circ} 40^{\prime} 0$ & Lopes et al. (2016) \\
\hline Fazenda Piraputanga & $16^{\circ} 28^{\prime} \mathrm{S}, 56^{\circ} 08^{\prime} 0$ & Tubelis \& Tomas (2003a) \\
\hline Fazenda Pouso Alegre & $16^{\circ} 30^{\prime} \mathrm{S}, 56^{\circ} 44^{\prime} 0$ & Nunes (2011a), Xeno-Canto (2021), WikiAves (2021) \\
\hline Fazenda Quebracho/Porto Quebracho & $21^{\circ} 50^{\prime} S, 57^{\circ} 53^{\prime} 0$ & Tubelis \& Tomas (2003a), Straube et al. (2006a), this study (APN, November 2011) \\
\hline Fazenda Rabicho & $18^{\circ} 59^{\prime} S, 57^{\circ} 37^{\prime} 0$ & Tubelis \& Tomas (2003a) \\
\hline Fazenda Rancharia & $18^{\circ} 34^{\prime} S, 55^{\circ} 50^{\prime} 0$ & This study (WMT, 2007) \\
\hline Fazenda Retirinho & $19^{\circ} 59^{\prime} \mathrm{S}, 56^{\circ} 02^{\prime} 0$ & Tubelis \& Tomas (2003a), this study (APN, August 2011) \\
\hline Fazenda Rio Alegre & $17^{\circ} 08^{\prime} S, 56^{\circ} 53^{\prime} 0$ & Tubelis \& Tomas, (2003a) \\
\hline Fazenda Rio Claro & $16^{\circ} 37^{\prime} S, 56^{\circ} 44^{\prime} 0$ & Tubelis \& Tomas (2003a) \\
\hline Fazenda Rio Negro & $19^{\circ} 30^{\prime} \mathrm{S}, 56^{\circ} 17^{\prime} 0$ & Tubelis \& Tomas, 2003a), Donatelli (2005), Cestari (2006a, b), Donatelli et al. $(2014,2017)$ \\
\hline Fazenda San Francisco & $20^{\circ} 05^{\prime} \mathrm{S}, 56^{\circ} 36^{\prime} 0$ & Melo et al. (2007), Melo \& Teribeli (2008), WikiAves (2021), this study (AVM, 2005) \\
\hline Fazenda Santa Cruz & $17^{\circ} 04^{\prime} S, 56^{\circ} 54^{\prime} 0$ & Tubelis \& Tomas (2003a) \\
\hline Fazenda Santa Emília & $19^{\circ} 30^{\prime} \mathrm{S}, 55^{\circ} 33^{\prime} 0$ & Pivatto et al. (2008) \\
\hline Fazenda Santa Inês & $16^{\circ} 30^{\prime} \mathrm{S}, 56^{\circ} 45^{\prime} 0$ & Tubelis \& Tomas (2003a) \\
\hline Fazenda Santa Isabel & $17^{\circ} 10^{\prime} \mathrm{S}, 57^{\circ} 01^{\prime} 0$ & Tubelis \& Tomas (2003a) \\
\hline Fazenda Santa Lúcia & $21^{\circ} 02^{\prime} S, 57^{\circ} 44^{\prime} 0$ & This study (APN, November 2011) \\
\hline Fazenda Santa Teresa/Pixaim & $16^{\circ} 45^{\prime} S, 56^{\circ} 52^{\prime} 0$ & Xeno-Canto (2021), WikiAves (2021) \\
\hline Fazenda Santa Vergínia & $22^{\circ} 01^{\prime} S, 57^{\circ} 54^{\prime} 0$ & This study (APN, November 2011) \\
\hline Fazenda Santana & $19^{\circ} 37^{\prime} \mathrm{S}, 55^{\circ} 36^{\prime} 0$ & Nunes et al. (2008), this study (APN, September 2005 and January 2016) \\
\hline Fazenda Santana do Paiaguás & $18^{\circ} 03^{\prime} S, 56^{\circ} 31^{\prime} 0$ & This study (WMT, 2007) \\
\hline Fazenda Santo Antônio das Lendas & $16^{\circ} 39^{\prime} \mathrm{S}, 57^{\circ} 50^{\prime} 0$ & Tubelis \& Tomas (2003a), Lopes et al. (2016) \\
\hline Fazenda Santo Expedito & $19^{\circ} 06^{\prime} S, 56^{\circ} 43^{\prime} 0$ & Nunes (2015) \\
\hline Fazenda São Bento & $19^{\circ} 29^{\prime} \mathrm{S}, 56^{\circ} 59^{\prime} 0$ & Araújo (2001), Yabe et al. (2010), Severo-Neto et al. (2015), this study (RRL, since 2010) \\
\hline Fazenda São Francisco & $16^{\circ} 54^{\prime} S, 56^{\circ} 15^{\prime} 0$ & WikiAves (2021) \\
\hline Fazenda São Francisco do Paiaguás & $17^{\circ} 46^{\prime} \mathrm{S}, 55^{\circ} 37^{\prime} 0$ & This study (WMT, 2007) \\
\hline Fazenda São Francisco do Tereré & $21^{\circ} 19^{\prime} S, 57^{\circ} 50^{\prime} 0$ & Naumburg (1935) \\
\hline Fazenda São João & $16^{\circ} 56^{\prime} S, 56^{\circ} 37^{\prime} 0$ & Tubelis \& Tomas (2003a) \\
\hline Fazenda São José do Piquiri & $17^{\circ} 14^{\prime} S, 56^{\circ} 34^{\prime} 0$ & Tubelis \& Tomas (2003a) \\
\hline Fazenda São Luis & $18^{\circ} 09^{\prime} S, 57^{\circ} 01^{\prime} 0$ & Nunes (2011b) \\
\hline Fazenda São Pedro & $16^{\circ} 22^{\prime} \mathrm{S}, 56^{\circ} 22^{\prime} 0$ & Tubelis \& Tomas (2003a) \\
\hline Fazenda São Roque & $19^{\circ} 10^{\prime} S, 56^{\circ} 42^{\prime} 0$ & This study (RRL, September 2017) \\
\hline Fazenda Taboco & $22^{\circ} 04^{\prime} S, 55^{\circ} 38^{\prime} 0$ & This study (APN, September 2005 and January 2006) \\
\hline Fazenda Tarumã & $19^{\circ} 05^{\prime} S, 57^{\circ} 06^{\prime} 0$ & Tubelis \& Tomas (2003a) \\
\hline Fazenda Tarumã do Nabileque & $20^{\circ} 17^{\prime} S, 57^{\circ} 38^{\prime} 0$ & Straube et al. (2006b) \\
\hline Fazenda Tereré & $21^{\circ} 25^{\prime} S, 57^{\circ} 48^{\prime} 0$ & This study (APN, November 2011) \\
\hline Fazenda Terra Preta & $20^{\circ} 24^{\prime} S, 57^{\circ} 21^{\prime} 0$ & Straube et al. (2006b) \\
\hline Firme & $21^{\circ} 12^{\prime} S, 57^{\circ} 26^{\prime} 0$ & Straube et al. (2006b) \\
\hline Forte Coimbra & $19^{\circ} 55^{\prime} S, 57^{\circ} 47^{\prime} 0$ & Tubelis \& Tomas (2003a) \\
\hline Foz do Rio Apa & $22^{\circ} 05^{\prime} S, 57^{\circ} 59^{\prime} 0$ & Straube et al. (2006a) \\
\hline Foz do Rio Jauru/Cáceres & $16^{\circ} 20^{\prime} S, 57^{\circ} 46^{\prime} 0$ & WikiAves (2021) \\
\hline Hotel Porto Jofre & $1721^{\circ} \mathrm{S}, 56^{\circ} 46^{\prime} 0$ & WikiAves (2021) \\
\hline Ilha do Nabileque & $20^{\circ} 22^{\prime} S, 57^{\circ} 44^{\prime} 0$ & This study (APN, November 2011) \\
\hline Ilha dos Bugres/Passo do Bugre & $19^{\circ} 47^{\prime} S, 57^{\circ} 39^{\prime} 0$ & Tubelis \& Tomas (2003a) \\
\hline Joselândia & $16^{\circ} 32^{\prime} S, 56^{\circ} 09^{\prime} 0$ & WikiAves (2021) \\
\hline Lagoa de Chacororé & $16^{\circ} 02^{\prime} S, 57^{\circ} 43^{\prime} 0$ & Lopes et al. (2016) \\
\hline Margem do Rio Paraguai & $21^{\circ} 42^{\prime} S, 57^{\circ} 54^{\prime} 0$ & Benites et al. (2017) \\
\hline
\end{tabular}




\begin{tabular}{|c|c|c|}
\hline Localities & Coordinates & Reference \\
\hline Miranda & $20^{\circ} 14^{\prime} S, 56^{\circ} 22^{\prime} 0$ & Tubelis \& Tomas (2003a) \\
\hline Moquém & $16^{\circ} 23^{\prime} S, 56^{\circ} 16^{\prime} 0$ & Vasconcelos et al. (2008) \\
\hline Morro/llha do Puga & $19^{\circ} 37^{\prime} \mathrm{S}, 57^{\circ} 30^{\prime} 0$ & Tubelis \& Tomas (2003a) \\
\hline Morro Pão de Açúcar & $21^{\circ} 26^{\prime} S, 57^{\circ} 53^{\prime} 0$ & Tubelis \& Tomas (2003a), this study (APN \& WMT, November 2011) \\
\hline Paiaguás & $18^{\circ} 11^{\prime} S, 55^{\circ} 33^{\prime} 0$ & This study (WMT, 2007) \\
\hline Pantanal do Abobral & $19^{\circ} 27^{\prime} S, 57^{\circ} 03^{\prime} 0$ & Tubelis \& Tomas (2003a) \\
\hline Paratudal & $19^{\circ} 35^{\prime} S, 57^{\circ} 02^{\prime} 0$ & Amaral \& Ragusa-Netto (2008) \\
\hline Parque de exposições, Poconé & $16^{\circ} 19^{\prime} S, 56^{\circ} 32^{\prime} 0$ & Tubelis \& Tomas (2003a) \\
\hline Parque Estadual Encontro das Águas & $17^{\circ} 07^{\prime} S, 56^{\circ} 41^{\prime} 0$ & WikiAves (2021) \\
\hline PARNA Pantanal Matogrossense & $17^{\circ} 51^{\prime} \mathrm{S}, 57^{\circ} 25^{\prime} 0$ & Jesus \& Silva (2003), WikiAves (2021) \\
\hline Passo do Lontra & $19^{\circ} 34^{\prime} S, 57^{\circ} 02^{\prime} 0$ & Tubelis \& Tomas (2003a), Nunes et al. (2010), Xeno-Canto (2021), WikiAves (2021) \\
\hline Pirizal & $16^{\circ} 14^{\prime} S, 56^{\circ} 23^{\prime} 0$ & Pinho (2005), Signor \& Pinho (2010), Evangelista et al. (2010) \\
\hline Poconé & $16^{\circ} 15^{\prime} \mathrm{S}, 56^{\circ} 37^{\prime} 0$ & Tubelis \& Tomas (2003a), this study (RSM, 2012) \\
\hline Ponte do Rio Nabileque & $20^{\circ} 21^{\prime} S, 57^{\circ} 38^{\prime} 0$ & Straube et al. (2006b) \\
\hline Ponte do Rio Naitaca & $20^{\circ} 37^{\prime} S, 57^{\circ} 34^{\prime} 0$ & This study (APN, November 2011) \\
\hline Porto Conceição & $17^{\circ} 08^{\prime} S, 57^{\circ} 21^{\prime} 0$ & Lopes et al. (2016) \\
\hline Porto Cercado & $16^{\circ} 30^{\prime} S, 56^{\circ} 22^{\prime} 0$ & Tubelis \& Tomas (2003a) \\
\hline Porto da Fazenda & $16^{\circ} 27^{\prime} S, 57^{\circ} 07^{\prime} 0$ & Tubelis \& Tomas (2003a) \\
\hline Porto da Manga & $19^{\circ} 15^{\prime} \mathrm{S}, 57^{\circ} 14^{\prime} 0$ & Sick (1997), Nunes et al. (2010), WikiAves (2021) \\
\hline Porto de Corumbá & $18^{\circ} 59^{\prime} S, 57^{\circ} 39^{\prime} 0$ & WikiAves (2021) \\
\hline Porto do Alegre & $17^{\circ} 37^{\prime} S, 56^{\circ} 57^{\prime} 0$ & This study (WMT, 2007) \\
\hline Porto Esperança & $19^{\circ} 36^{\prime} \mathrm{S}, 57^{\circ} 26^{\prime} 0$ & Naumburg et al. (1930), Tubelis \& Tomas (2003a), WikiAves (2021) \\
\hline Porto Faia & $18^{\circ} 22^{\prime} S, 57^{\circ} 21^{\prime} 0$ & Naumburg et al. (1930) \\
\hline Porto Jofre/Santa Rosa & $17^{\circ} 21^{\prime} S, 56^{\circ} 46^{\prime} 0$ & Tubelis \& Tomas (2003a) \\
\hline Porto Murtinho & $21^{\circ} 41^{\prime} S, 57^{\circ} 52^{\prime} 0$ & Tubelis \& Tomas (2003a), Straube et al. (2006a) \\
\hline Posto do IBAMA, Transpantaneira & $16^{\circ} 21^{\prime} \mathrm{S}, 56^{\circ} 38^{\prime} 0$ & Tubelis \& Tomas (2003a) \\
\hline Pousada Pantaneiro & $16^{\circ} 56^{\prime} S, 56^{\circ} 53^{\prime} 0$ & Xeno-Canto (2021) \\
\hline Pousada Pequi & $20^{\circ} 11^{\prime} S, 55^{\circ} 55^{\prime} 0$ & WikiAves (2021) \\
\hline Pousada Piuval & $16^{\circ} 23^{\prime} S, 56^{\circ} 35^{\prime} 0$ & Emanuel (2013), Xeno-Canto (2021), this study (DMM0 \& BC, 2010 to 2019) \\
\hline Pousada Refúgio da llha & $20^{\circ} 13^{\prime} \mathrm{S}, 56^{\circ} 34^{\prime} 0$ & Ribas et al. (2011), WikiAves (2021) \\
\hline Pousada Rio Mutum & $16^{\circ} 20^{\prime} S, 55^{\circ} 51^{\prime} 0$ & WikiAves (2021) \\
\hline Pousada Xaraés & $19^{\circ} 29^{\prime} S, 56^{\circ} 57^{\prime} 0$ & Nunes et al. (2010) \\
\hline Reserva Kadiwéu & $20^{\circ} 39^{\prime} S, 57^{\circ} 29^{\prime} 0$ & Straube et al. (2006a), this study (APN, November 2011) \\
\hline Retiro Carandá & $21^{\circ} 47^{\prime} S, 57^{\circ} 34^{\prime} 0$ & WikiAves (2021) \\
\hline Retiro Novo & $16^{\circ} 22^{\prime} S, 56^{\circ} 18^{\prime} 0$ & Vasconcelos et al. (2008), Nabuco et al. (2018) \\
\hline Riacho Sanga Funda & $22^{\circ} 04^{\prime} S, 57^{\circ} 34^{\prime} 0$ & Straube et al. (2006a) \\
\hline Rio Nabileque & $20^{\circ} 44^{\prime} S, 57^{\circ} 43^{\prime} 0$ & Straube et al. (2006b) \\
\hline Rio Paraguai-Mirim & $19^{\circ} 00^{\prime} S, 57^{\circ} 25^{\prime} 0$ & Tubelis \& Tomas (2003a) \\
\hline Rio Piquiri & $17^{\circ} 56^{\prime} \mathrm{S}, 56^{\circ} 12^{\prime} 0$ & This study (WMT, 2007) \\
\hline Rio São Lourenço/Cuiabá & $17^{\circ} 29^{\prime} S, 56^{\circ} 52^{\prime} 0$ & Tubelis \& Tomas (2003a) \\
\hline Rio Tarumã & $21^{\circ} 32^{\prime} S, 57^{\circ} 49^{\prime} 0$ & Straube et al. (2006a) \\
\hline rios Miranda/Abobral & $19^{\circ} 34^{\prime} \mathrm{S}, 57^{\circ} 01^{\prime} 0$ & Tubelis \& Tomas (2003a), Nunes et al. (2010) \\
\hline rios Vermelho/Miranda & $19^{\circ} 36^{\prime} S, 56^{\circ} 56^{\prime} 0$ & Tubelis \& Tomas (2003a) \\
\hline Rodovia BR-267/MS & $21^{\circ} 41^{\prime} S, 57^{\circ} 51^{\prime} 0$ & Benites et al. (2017) \\
\hline Rodovia MS-195 & $20^{\circ} 04^{\prime} S, 57^{\circ} 32^{\prime} 0$ & This study (WMT, November 2011) \\
\hline Rodovia MS-325 & $20^{\circ} 01^{\prime} S, 57^{\circ} 20^{\prime} 0$ & This study (WMT, november 2011) \\
\hline Rodovia Ramon Gomes (marginal stretch to Canal do Tamengo) & $19^{\circ} 00^{\prime} S, 57^{\circ} 40^{\prime} 0$ & WikiAves (2021) \\
\hline RPPN SESC & $16^{\circ} 39^{\prime} \mathrm{S}, 56^{\circ} 16^{\prime} 0$ & Tubelis \& Tomas (2003a), Brandão et al. (2011), Ubaid \& Antas (2013), Ubaid et al. (2010), WikiAves (2021) \\
\hline Salobra & $20^{\circ} 11^{\prime} \mathrm{S}, 56^{\circ} 30^{\prime} 0$ & Tubelis \& Tomas (2003a) \\
\hline Santo Antônio (antiga usina) & $15^{\circ} 52^{\prime} S, 56^{\circ} 04^{\prime} 0$ & Tubelis \& Tomas (2003a) \\
\hline Santo Antônio do Leverger & $15^{\circ} 59^{\prime} \mathrm{S}, 56^{\circ} 07^{\prime} 0$ & Xeno-Canto (2021), WikiAves (2021) \\
\hline Sudeste da Nhecolândia & $19^{\circ} 18^{\prime} \mathrm{S}, 56^{\circ} 06^{\prime} 0$ & Tubelis \& Tomas (2003a) \\
\hline Transpantaneira & $16^{\circ} 24^{\prime} \mathrm{S}, 56^{\circ} 40^{\prime} 0$ & Tubelis \& Tomas (2003a) \\
\hline Transpantaneira (10 km South of Poconé) & $16^{\circ} 36^{\prime} \mathrm{S}, 56^{\circ} 59^{\prime} 0$ & Xeno-Canto (2021) \\
\hline Transpantaneira (stretch between Rio Bento Gomes and Pixaim) & $16^{\circ} 35^{\prime} \mathrm{S}, 56^{\circ} 44^{\prime} 0$ & Tubelis \& Tomas (2003a) \\
\hline Tucum & $16^{\circ} 30^{\prime} S, 57^{\circ} 48^{\prime} 0$ & Lopes et al. (2016) \\
\hline Vazante do Capivari & $18^{\circ} 14^{\prime} S, 56^{\circ} 12^{\prime} 0$ & Serrano $(2010)$ \\
\hline
\end{tabular}

Acronyms of authors in alphabetical order: Alessandro Pacheco Nunes (APN), Alyson Vieira de Melo (AVM), Angélica Vilas Boas da Frota (AVBF), Breno Dias Vitorino (BDV), Carlos Braulio (CB), Dalci Maurício Miranda de Oliveira (DMM0), Fernando Costa Straube (FCS), Gabriel Oliveira de Freitas (GOF), Maria Antonietta Castro Pivatto (MACP), Maristela Benites (MB), Rafael Augusto Ducel de Souza (RADS), Reginaldo José Donatelli (RJD), Renato Soares Moreira (RSM), Rudi Ricardo Laps (RRL), Simome Mamede (SM), Sérgio Roberto Posso (SRP) and Walfrido Moraes Tomas (WMT). 
Sites and records (regions without precision of sampled site) of birds species in the Pantanal wetland, followed by their sources of information.

\begin{tabular}{|c|c|}
\hline Localities & Reference \\
\hline Flood area of the Rio Bento Gomes & WikiAves (2021) \\
\hline Barra do Rio Paraguai/São Lourenço & WikiAves (2021) \\
\hline Barão de Melgaço & WikiAves (2021) \\
\hline BR-262 (between Miranda and Corumbá) & WikiAves (2021) \\
\hline Cáceres & WikiAves (2021) \\
\hline Corumbá & Vasconcelos \& Hoffmann (2006), WikiAves (2021) \\
\hline Coxim & WikiAves (2021) \\
\hline Cuiabá & WikiAves (2021) \\
\hline Estrada Parque Pantanal Sul & WikiAves (2021) \\
\hline Estrada Parque Pantanal Sul (between Curva do Leque and Porto da Manga) & WikiAves (2021) \\
\hline Fazenda Miranda & VertNet (2021) \\
\hline Fazenda Nova Esperança & WikiAves (2021) \\
\hline Foz do Rio Negro & Nascimento et al. (2000) \\
\hline Miranda & WikiAves (2021) \\
\hline Nhecolândia & Tubelis \& Tomas (2003a), Serrano (2010) \\
\hline Nordeste do Pantanal & Tubelis \& Tomas (2003a) \\
\hline Pantanal & Cintra (2014) \\
\hline Pantanal da Nhecolândia & WikiAves (2021) \\
\hline Pantanal do Abobral & WikiAves (2021) \\
\hline Poconé & WikiAves (2021) \\
\hline Porto Murtinho & WikiAves (2021) \\
\hline Região do Rio Negro & Tubelis \& Tomas (2003a) \\
\hline Rio São Lourenço e Cuiabá & Tubelis \& Tomas (2003a) \\
\hline Rio Taquari & Tubelis \& Tomas (2003a) \\
\hline Rio Verde de Mato Grosso & WikiAves (2021) \\
\hline Rodovia Transpantaneira & WikiAves (2021) \\
\hline Rodovia Transpantaneira (between Poconé and Porto Jofre) & Tubelis \& Tomas (2003a) \\
\hline Salinas do Rio Negro & Serrano (2010) \\
\hline Santo Antônio do Leverger & WikiAves (2021) \\
\hline
\end{tabular}

